Andrews University

Digital Commons @ Andrews University

2018

\title{
The Impact of Media Influence About Hair Texture on Internalized Racial Oppression, Ethnic Identity, and Self-Efficacy
}

Kristy L. La Mar

Andrews University, kristy@andrews.edu

Follow this and additional works at: https://digitalcommons.andrews.edu/dissertations

Part of the African American Studies Commons, and the Women's Studies Commons

\section{Recommended Citation}

La Mar, Kristy L., "The Impact of Media Influence About Hair Texture on Internalized Racial Oppression, Ethnic Identity, and Self-Efficacy" (2018). Dissertations. 1660.

https://digitalcommons.andrews.edu/dissertations/1660

https://dx.doi.org/10.32597/dissertations/1660

This Dissertation is brought to you for free and open access by the Graduate Research at Digital Commons @ Andrews University. It has been accepted for inclusion in Dissertations by an authorized administrator of Digital Commons@ Andrews University. For more information, please contact repository@andrews.edu. 


\begin{abstract}
THE IMPACT OF MEDIA INFLUENCE ABOUT HAIR TEXTURE ON INTERNALIZED RACIAL OPPRESSION, ETHNIC IDENTITY, AND SELF-EFFICACY
\end{abstract}

Kristy L. La Mar

Chair: Carole Woolford-Hunt 


\title{
ABSTRACT OF GRADUATE STUDENT RESEARCH
}

Dissertation

\author{
Andrews University
}

School of Education

\section{Title: THE IMPACT OF MEDIA INFLUENCE ABOUT HAIR TEXTURE ON INTERNALIZED RACIAL OPPRESSION, ETHNIC IDENTITY, AND SELF-EFFICACY}

Name of researcher: Kristy L. La Mar

Name and degree of faculty chair: Carole Woolford-Hunt, Ph.D.

Date completed: July 2018

\section{Problem}

African American women and matters of importance pertaining to African American women are understudied and lacking within research. Understanding the ways in which majority culture media influences aspects related to women of color, especially those pertaining to hair texture, and their impact is essential to bring about awareness and mobilize changes that will positively affect the lives of Black women.

Representation matters. When minority women of any culture value the majority culture they tend to devalue themselves. Representation matters and is important because if selfesteem, worth, goodness, and virtue is only portrayed in majority culture aesthetics, internalization of those influential concepts of good and bad takes place, and that begins to transform and seep into every orifice of the Black women's life. The impact of beauty 
on power and economic and social mobility leaves many African American women disenfranchised and disempowered because the definition of beauty, especially as it pertains to hair texture, is narrow and the standard of beauty by cultural and societal standards denies benefits, economic, social, education, vocation, and otherwise, to those who are not defined as such.

\section{Method}

Participants completed surveys that measured a) media influence about hair texture, b) internalized racial oppression, c) ethnic identity, and d) self-efficacy. This work employed structural equation modeling to test the hypothesized model for the impact of media influence about hair texture on internalized racial oppression, ethnic identity, and self-efficacy; as well as three within group models for ethnic identification, hair texture, and skin tone.

\section{Results}

Structural equation modeling indicated that the original hypothesized theoretical model was a poor fit to the data. Therefore, the model was revised on the basis of theory and modification indices. The revised model revealed that media influence about hair texture had a positive direct effect on internalized racial oppression. Media influence about hair texture had a positive direct effect on ethnic identity but also a negative direct effect on ethnic identity as it pertains to affirmation, commitment, and belonging. Media influence about hair texture had a negative direct effect on self-efficacy. The revised model was also well fitted to the between groups ethnic identification, hair texture, and skin tone with the impact of media influence about hair texture on internalized racial oppression being two times greater in African American women than black women. 


\section{Conclusions}

This study added to the limited body of literature concerning African American women and the role that media influence plays on internalized racial oppression, aspects of ethnic identity, and self-efficacy. It suggests that high media influence about hair texture contribute to high levels of internalized racial oppression; low levels of ethnic affirmation, commitment, and belonging; and low levels of self-efficacy.

It also confirmed that media influence about hair texture on internalized racial oppression, ethnic identity, and self-efficacy impacts African American and Black ethnically identified women, hair textures, and skin tones differently. In particular, this study suggests that higher levels of media influence increases minorities' adoption of racist messages; higher levels of media influence lower one's confidence of success and achievement; and higher levels of media influence lower minorities' desire to affirm, belong, and commit to their ethnic group. 
Andrews University

School of Education

\title{
THE IMPACT OF MEDIA INFLUENCE ABOUT HAIR TEXTURE ON INTERNALIZED RACIAL OPRESSION, ETHNIC IDENTITY, AND SELF-EFFICACY
}

\author{
A Dissertation \\ Presented in Partial Fulfillment \\ of the Requirements for the Degree \\ Doctor of Philosophy
}

by

Kristy L. La Mar

July 2018 
(C) Copyright by Kristy L. La Mar 2018

All Rights Reserved 


\title{
THE IMPACT OF MEDIA INFLUENCE ABOUT HAIR TEXTURE ON INTERNALIZED RACIAL OPPRESSION, ETHNIC IDENTITY, AND SELF-EFFICACY
}

\author{
A dissertation \\ presented in partial fulfillment \\ of the requirements for the degree \\ Doctor of Philosophy
}

by

Kristy L. La Mar

APPROVAL BY THE COMMITTEE:

Chair: Carole Woolford-Hunt

Member: Nadia Nosworthy

Member: Jimmy Kijai

External: Lionel Matthews
Dean, School of Education

Robson Marinho 
Dedicated to James \& Veronica, my ever-loving and supportive parents.

Papa, thank you for always taking me to the Word, where therein I found life and hope beyond my circumstances. 


\section{TABLE OF CONTENTS}

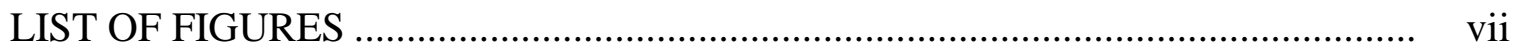

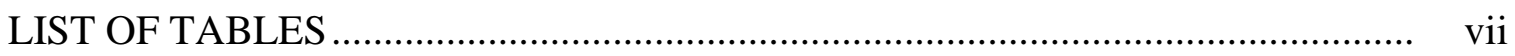

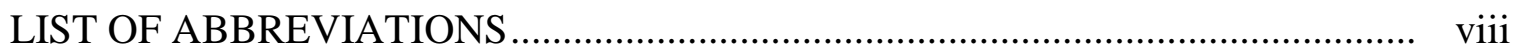

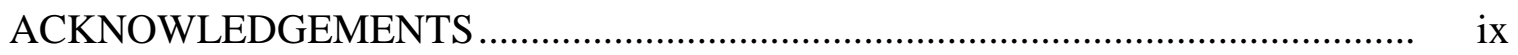

Chapter

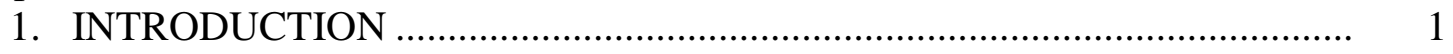

Background of the Problem ....................................................................... 1

Statement of the Problem........................................................................... 3

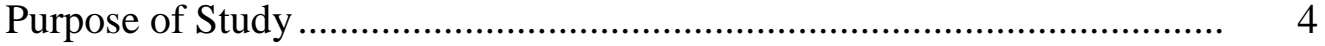

Hypothesis and Research Questions ........................................................ 4

Rationale ……................................................................................ 5

Conceptual Framework ........................................................................... 7

Importance of the Study ........................................................................

Definition of Terms ......................................................................... 10

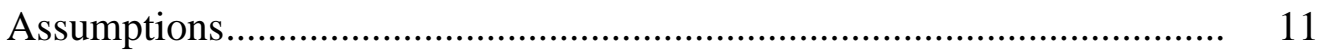

General Methodology ....................................................................... 12

Limitations ............................................................................. 13

Delimitations ............................................................................ 14

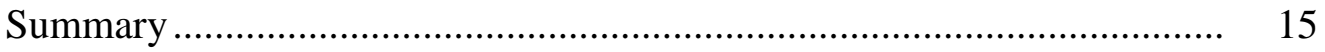

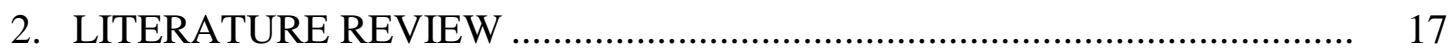

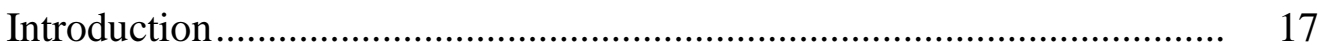

Theoretical Framework .................................................................... 18

African American Hair History ………………………............................. 19

Hair Texture Consciousness Among African Americans ........................... 24

Media Influence ............................................................................. 35

Internalized Racial Oppression ............................................................. 41

Ethnic Identity ................................................................................. 44

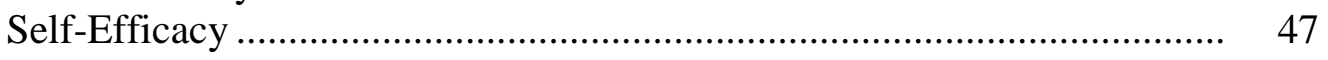

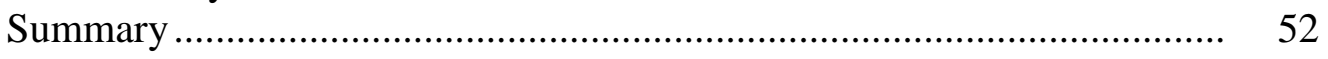

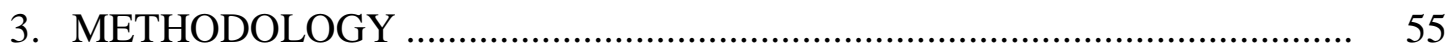




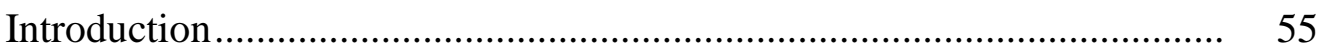

Research Design............................................................................ 55

Population and Sample ....................................................................... 57

Hypothesis and Research Questions .................................................... 58

Definitions of Variables ...................................................................... 58

Exogenous Variables .................................................................. 58

Endogenous Variables ................................................................. 59

Instrumentation .................................................................... 59

The Sociocultural Attitudes Toward Appearance Questionnaire-3

Modified .............................................................................. 61

Internalized Racial Oppression Scale ……………………................ 62

The Multigroup Ethnic Identity Measure …………………................. 63

The General Self Efficacy Scale ........................................................ 65

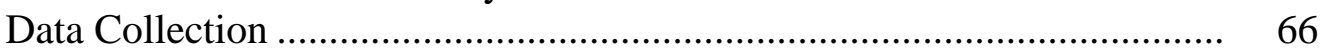

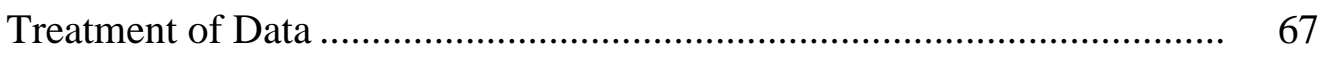

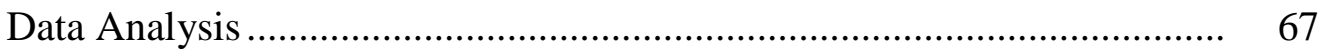

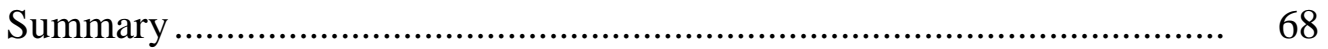

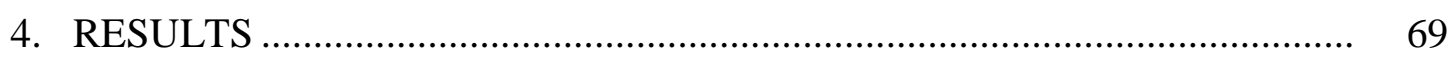

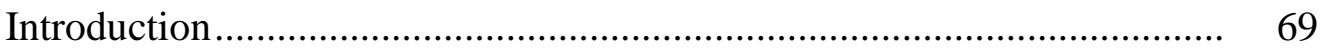

Description of Sample.......................................................................... 69

Instrument Reliability ........................................................................ 71

Variables Description........................................................................ 72

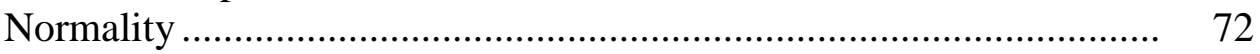

Variable Means and Standard Deviations ............................................ 73

Zero-Order Correlations.................................................................. 75

Hypothesis Testing............................................................................ $\quad 77$

Research Question One ................................................................. 77

Original Model ......................................................................... 77

Fitted Model....................................................................... 80

Explanation of the Fitted Model ................................................ 82

Research Question Two ................................................................. 85

Ethnic Identification............................................................... 85

Hair Texture ....................................................................... 88

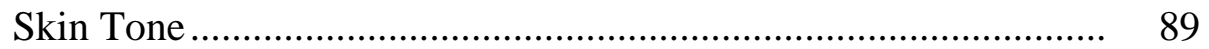

Summary ……........................................................................... 91

5. SUMMARY, DISCUSSION, AND IMPLICATIONS .................................... 93

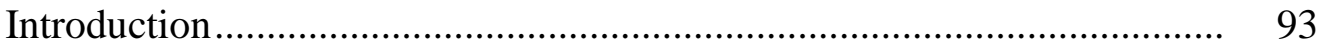

Purpose of the Study ........................................................................... 93

Summary of the Literature Review ........................................................... 94

Methodology ................................................................................. 96

Findings and Discussion ...................................................................... 97

Respondents' Demographic Characteristics ........................................ 97 
Zero-Order Correlations.................................................................. 98

Research Question One and Model Fit ................................................ 98

Media Influence About Hair Texture and Internalized Racial Oppression .................................................................... 99

Media Influence About Hair Texture and Ethnic Identity ............. 100

Media Influence About Hair Texture and Ethnic Identity

Affirmation, Belonging, and Commitment............................... 101

Media Influence About Hair Texture and Self-Efficacy................. 102

Internalized Racial Oppression and Ethnic Identity ...................... 103

Ethnic Identity and Self-Efficacy................................................. 103

Research Question Two and Fitted Model......................................... 104

Ethnic Identification................................................................. 104

Hair Texture ....................................................................... 106

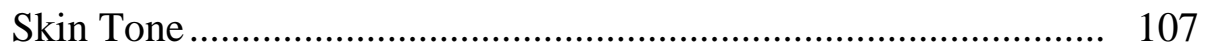

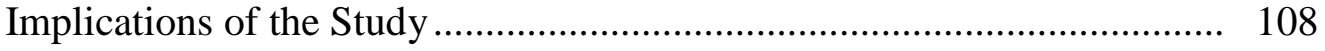

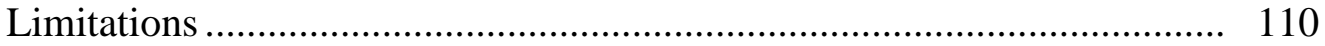

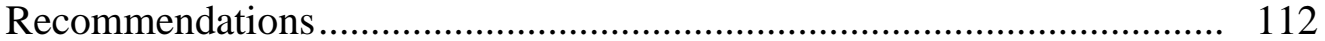

Recommendations for Practice ........................................................ 113

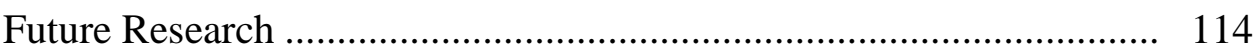

Appendix

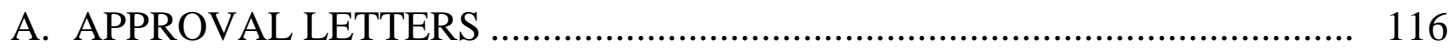

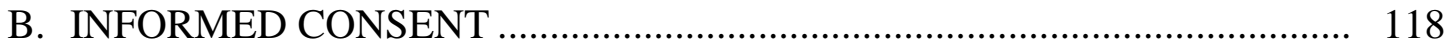

C. DEMOGRAPHIC \& HAIR QUESTIONNAIRES ........................................ 120

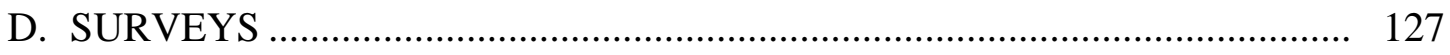

E. OUTPUT FOR FITTED MODEL ........................................................... 135

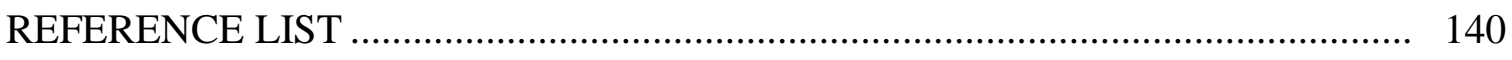

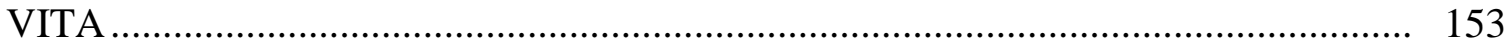




\section{LIST OF FIGURES}

1. Hypothesized Model of the Relationship Between Variables ........................ 5

2. Respecified Model ......................................................................... 81

3. Respecified Model: African American ................................................... 86

4. Respecified Model: Black..................................................................... 86

5. Respecified Model: Hair Texture 1 to 4 ................................................. 90

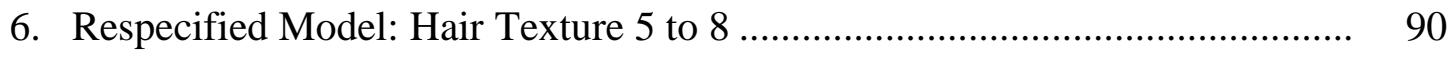

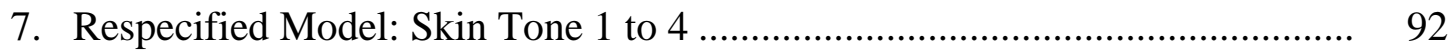

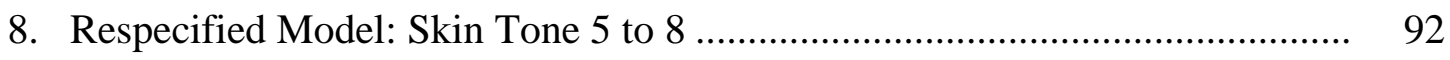

\section{LIST OF TABLES}

1. Conceptual Definitions of Variables and Source of Associated Survey

Items......

2. Respondents' Demographic Characteristics .......................................... 70

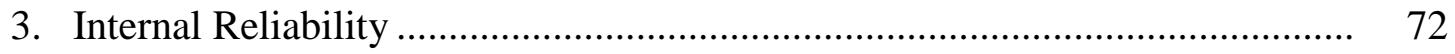

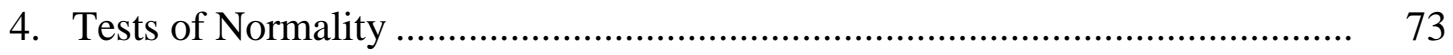

5. Variable Means and Standard Deviations ................................................. 74

6. Zero-Order Correlations.......................................................................... 76

7. Chi-Square and Goodness of Fit Indices for Hypothesized and Respecified Models .................................................................... 78

8. Standardized Regression Weights of Variables Between Groups .................. 79 


\section{LIST OF ABBREVIATIONS}

$\begin{array}{ll}\text { AA } & \text { African Americans } \\ \text { ABC } & \text { Affirmation, Belonging, Commitment } \\ \text { AMOS } & \text { Analysis of a Moment Structures } \\ \text { APA } & \text { Alteration of Physical Appearance } \\ \text { BRH } & \text { Belief in the Biased Representation of History } \\ \text { CFI } & \text { Comparative Fit Index } \\ \text { GFI } & \text { Goodness of Fit Index } \\ \text { GSE } & \text { General Self-Efficacy } \\ \text { HC } & \text { Hair Change } \\ \text { EI } & \text { Ethnic Identity } \\ \text { INS } & \text { Internalization of Negative Stereotypes } \\ \text { IRO } & \text { Internalized Racial Oppression } \\ \text { IROS } & \text { Internalized Racial Oppression Scale } \\ \text { MEIM } & \text { Multigroup Ethnic Identity Measure } \\ \text { MI } & \text { Media Influence } \\ \text { NAACP } & \text { National Association for the Advancement of Colored People } \\ \text { NFI } & \text { Normed Fit Index } \\ \text { RMSEA } & \text { Root Mean Square Error of Approximation } \\ \text { SATAQ3 } & \text { Sociocultural Attitude Toward Appearances } \\ \text { SEM } & \text { Structural Equation Modeling } \\ \text { SPSS24 } & \text { Statistical Package for the Social Sciences, } 24^{\text {th }} \text { Edition } \\ & \end{array}$




\section{ACKNOWLEDGMENTS}

First and foremost, I give ALL the praise, honor, and glory to God who is my EVERYTHING! The unmatched helper and my sustainer, for whom without, I would not be here today. I called upon Him and He heard me, and answered.

God used many people to support me in the completion of this dissertation. My parents, James and Veronica, provided me with love, encouragement, and a much-needed Christ-perspective when I questioned the possibilities of completing this task after my second neurosurgery. I am thankful for their many sacrifices. My sister, Tiffany, financially backed the enormous task of my data collection — truly an answered prayer. My brother, Junie, cheered me to the finish line. My niece, Allaraé, kept a huge smile on my face and joy in my heart.

Also, I am thankful for my cohort who became family to me, as well as my dear friends and colleagues who helped to make this dissertation a reality. A few in particular: Helen Rolle, who always spoke words of support and life to me, was my shoulder to lean on, and my weekly check-in partner; Esther Hooley, who offered help in countless ways; and Lauren Rugless, a friend like none other. Additionally, I am grateful to Jay Blackstone, Meagan Mingo, and Karen Benjamin, as well as Dr. Tamba-Kui Bailey, who kindly spoke with me about his instrument, the IROS, and provided the scale for use; Donel and Jill Wimberley, who generously opened up their home to me; Dr. Tevni E. Guerra Grajales, who graciously provided me with the help I needed to meet my personal 
deadlines; and Meridith Hart, who answered a million emails, phone calls, and emergency 'I need this ASAP' requests - the real MVP behind the scenes.

Last but not least, thank you to my dissertation committee: Dr. Woolford-Hunt, Dr. Nosworthy, and Dr. Kijai. This research topic was very important to me and near to my heart and my personal experiences. Dr. Woolford-Hunt, were it not for you sitting in the diversity gap, my research topic on hair texture would not have been possible. 


\section{CHAPTER 1}

\section{INTRODUCTION}

\section{Background of the Problem}

In a white dominant culture hair texture has been a source of shame and discomfort for African American (AA) women (Collins, 2002; Lakoff \& Scherr, 1984). Representations of beauty that are in opposition to natural, tightly coiled black hair often result in an internalization of images of beauty that have rejected natural hair as "natural" (Banks, 2000). This has led to the very real issue for Black women of natural hair discrimination. Straightening one's hair has been viewed as an "adaption to the reality of White supremacy" (Buchanan, 1993). African American women have internalized, in varying degrees, a Eurocentric ideal of beauty that has made hair-straightening (and weave wearing) imperative, which reinforces the notion that tightly coiled, kinky hair is unacceptable, unkempt, and unsightly (Collins, 2002; Leeds, 1994). Although the "Black is beautiful" message of the Civil Rights movement encouraged women to embrace their natural African hair, beauty products continue to be advertised specifically targeted toward "relaxing" naturally kinky hair. It has been speculated that products such as these can be psychologically damaging to AA women (West, 1995) because they send the message that these women are not acceptable as they are and should instead try to alter

their natural hair to become beautiful. Though it is noted by Banks (2000) that not all AA women straighten their hair from a desire to look white, the issue is "manageability," it 
begs the question why one has not learned to manage what in fact is genetically their own. The cry of "manageability" is also steeped in cultural oppression.

The media tells women what they should look like and who they should aspire to be, while at the same time often representing women in a stereotypical, biased, and discriminatory way. These messages directed at women are magnified for AA women due to the absence of images that actually look like them and reflect their cultural heritage (White, 2008). The lack of visibility of AA women and the stereotypical manner in which they are represented greatly limit one's conception of themselves and ideas about what it means to be beautiful and accepted (White, 2008). African American women who rarely experience the full range of images that truly represent them in diversity of skin tones, hair textures, shape and size will begin to think that who they are is not acceptable and definitely not what's "in" (White, 2008).

America's traditional and accepted standard of physical beauty is Eurocentric. The standards of beauty are created by and based on White women and their idealized features: straight blonde hair, fair skin, and thin bodies (Collins, 2002; Freedman, 1988). These normative beauty constructs set forth by the majority have been detrimental to the minority, especially AA women (White, 2008). When AA women are constantly exposed to images that highlight the dominant culture's ideas of beauty attributes, they will conclude not only that being "beautiful" is a female's proper role in society (White, 2008), but that one must aspire to and attain the dominant cultures definition of beauty to be accepted in that society. Advertisements for skin lighteners and hair straighteners marketed by white companies suggests to black women that only through changing 
physical features will persons' of African descent be afforded privilege and class mobility within AA communities and social acceptance by the dominant culture (Rooks, 1996).

\section{Statement of the Problem}

Since the Clark and Clark Doll Study—in which AA children internalized feelings of inferiority and damage to their self-esteem due to segregated schools in which they attributed negative characteristics such as bad or ugly to Black dolls that looked like themselves, and positive characteristics such as good and pretty to White doll that looked nothing like them-it is evident that representation matters (National Association for the Advancement of Colored People [NAACP], 2018). There is no difference when it comes to matters of hair texture, as hair is merely an extension of one's appearance, and is used as a means and method of classification in the world of beauty, looks, and appearance. This is why hair is a multi-billion dollar industry.

One's ability to successfully and strategically navigate the world depends largely on how they are perceived and a great deal of that is hinged upon how we look. Hair is no different from a nose, or lips, or skin color. Hair is a defining feature and what shape or texture it takes impacts how we see the world and how the world sees us.

Hair, like skin tone or complexion, will never be "blind" because one sees hair, even if one choose not to acknowledge that sight. People see hair shape, hair color, hair texture, hair length, and what they see in connection with all other attributes of physical appearance, will be the deciding factor of how they form an opinion and assessment of an individual. That in turn, leads to what category one belongs to, how one will be treated, spoken to, or what expectations, if any at all, will be placed on an individual solely based upon hair texture and associated attributes. Based on this, the minority, by majority 
influence will have a say as to what AA women, based upon hair, can and cannot achieve and arbitrarily prescribe limits and cap heights. Hair, like beauty, is power. Further research is needed to confirm the role of media influence (MI) on internalized racial oppression (IRO), ethnic identity (EI), and general self-efficacy (GSE).

\section{Purpose of the Study}

The purpose of this study is to examine the impact of MI about hair texture on IRO, EI, and GSE through model testing. The findings from this study will contribute to the very limited literature on AA women's issues regarding hair texture; provide knowledge of the relationship between MI and IRO, EI, and GSE; and validate hair attributes to bring about a conscious awareness that requires more inclusive and accurate representation of AA and Black women in the media's spotlight; and mobilize efforts to effect positive and substantial change.

\section{Hypothesis and Research Questions}

The main hypothesis of this study was that the reproduced covariance matrix proposed in the theoretical model (Figure 1) and the observed sample covariance matrices were equal. This hypothesis addressed the following research question: is the hypothesized theoretical model a good fit to the sample? As depicted, the theoretical model proposed a direct effect between MI and IRO, between MI and EI, and between MI and GSE. From the results of the main hypothesis, this study then sought to address the following research question: does ethnic identification, hair texture, or skin tone influence the fit of the empirical model? That is to say, does the fitted model relationship 


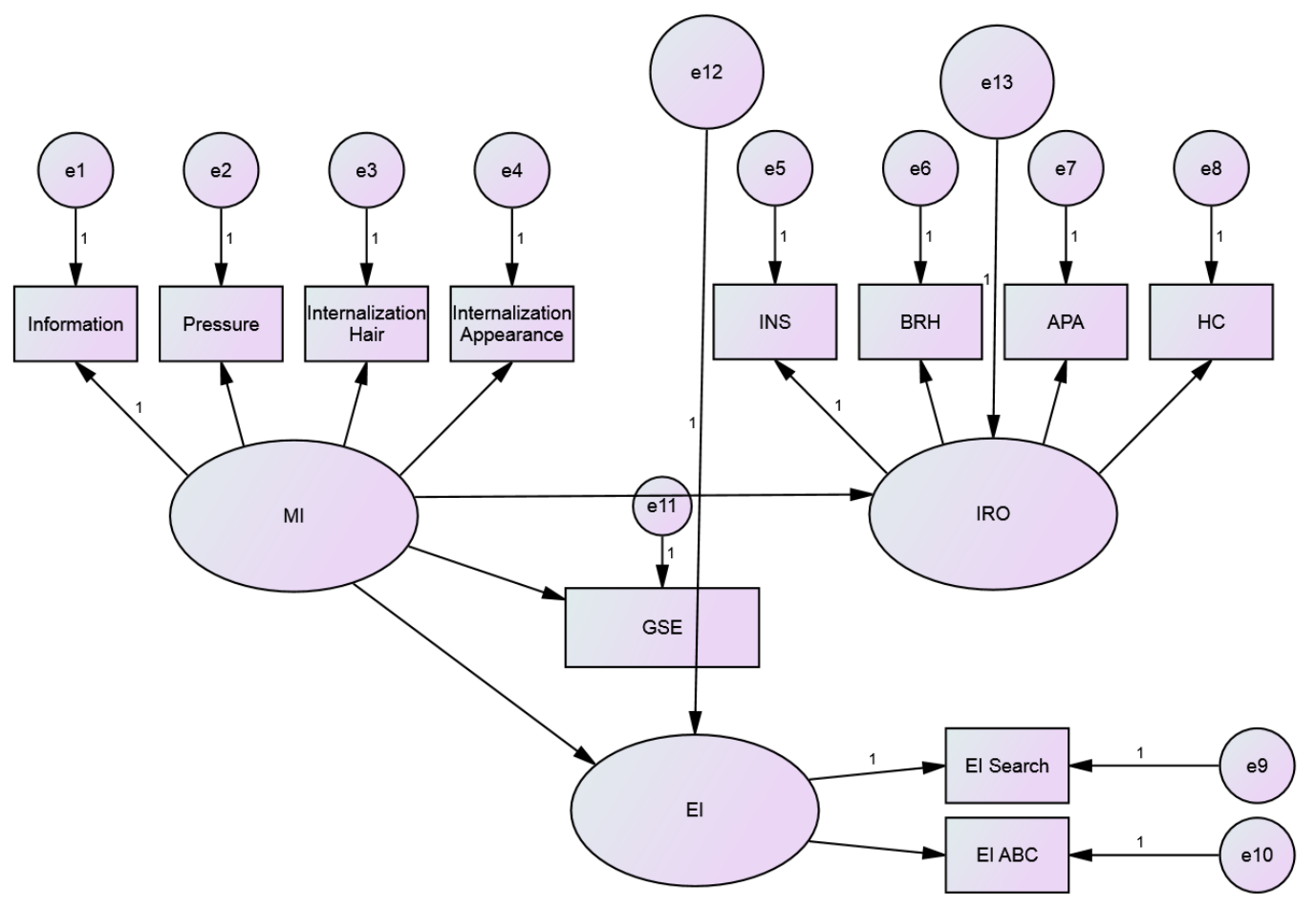

Figure 1. Hypothesized Model of the Relationship Between Variables.

between MI and IRO, between MI and EI, and between MI and GSE affect individuals differently depending on ethnicity, hair texture, or skin tone?

\section{Rationale}

In the United States, the Eurocentric beauty standard (whiteness and Western European features) (Lakoff \& Scherr, 1984; Wolf, 2002) is damaging to the AA women's self-esteem and produces unconscious IRO. Like all women, they are constantly bombarded with the notion that beauty should be their primary goal. But, as racial minorities, they are made to feel inadequate by an Anglo-American dominated cultural milieu that has historically both excluded them and distorted images of them in such a 
way that they themselves have come to associate those features (via internalized racism) stereotypically identified with their race with both negative personality and mental characteristics (Kaw, 1994). Beauty has become so normalized that it no longer seems social, political, or racial in nature but "our entire cultural ethos in the contemporary United States seethes of white beauty" (Hunter, 2013, p. 63). Media images influence AA women to assume that there is one "ideal" and standard of beauty to which they must subscribe. The majority of mass media reinforces racist conceptions by constructing historically distorted favorable images of Whites and negative images of people of color, in particular AAs (Allen, 2001). Hence why the media can be a major source of oppression for AAs. Because media mediums reflect societal values and beliefs they transmit a powerful message about certain members of society, such as AA women. Mainstream media informs us of each ethnic groups place on the totem pole of hierarchy within social structures and present 'societal attitudes' toward minority members (Fujioka, 2005). The negative depictions of AAs prevalent in mainstream media (Entman \& Rojecki, 2001) represent society's negative attitudes toward and perception of AAs.

Due to the strong consumption of media images (Riggott, 2013; Ward, 2004) AA women tend to accept the negative images of AAs as reality and the images of Caucasian women, subconsciously, as the beauty standard. The extent of the media's messages about hair texture as it relates to AA women's ethnicity, self-efficacy, and IRO is not known due to a lack of investigation on the topic. There is a lack of information on the specific role of MI and hair texture, and there is even less information on how MI about hair texture impacts IRO and self-efficacy of AA women. Little if any research has been based on the impact of the media portrayals of Caucasian American women on AA 
women as it relates to hair messages. Meanwhile, more negative images of AA women than Caucasian American women continue to appear in the media (Frisby, 2004; Hill, 1999; Thomas, 2006). African American women are bombarded with negative images of themselves (Allen, 2001; Woodard \& Mastin, 2005) while being presented with an unattainable beauty standard that has gone unquestioned or challenged for decades. As a result trauma, internalized racism, and low self-esteem affecting self-efficacy have resulted unbeknownst to the AA community.

It is critical that we examine MI about hair texture on IRO, EI, and GSE to determine their impact on viewers, particularly AA women, who are forming and critiquing their self-image based on beauty aesthetics portrayed in media mediums. Furthermore, in this respect the AA female population is overlooked, underserved, and a vulnerable population group.

\section{Conceptual Framework}

The Agenda-setting Theory serves as the theoretical framework for this study. The Agenda-setting theory, also known as The Agenda Setting Function of the Mass Media, which was first put forth by McCombs and Shaw (1972), describing the ability of media to influence the importance of topics to its viewers. This theory holds that the mass media has the powerful ability to bring issues to the attention of the public. That is, if a news or particular commercial item is covered frequently and prominently, the audience will regard the issue as more important. The basic claim is that as the media gives more attention to an issue, the public perceives the issue as important. The media sets the agenda, by implanting ideas into the minds of the general public, and the masses are affected based on the messages given. In this case, the media constantly portrays hair 
textures that are either long, silky, straight, or smooth—European—which most, if not all ethnicities have deemed "important" or to some extent better looking, more acceptable, more beautiful, and of great value.

Therefore, I hypothesized that MI contributes to IRO, problematic EI, and low GSE. I examined this hypothesized relationship on a whole and then within the study group using ethnic identification, hair texture, and skin tone variables. I conceptualized MI would have an impact on each of the variables: IRO, EI, and self-efficacy. In addition, I conceptualized that MI would have differing and greater effects on particular individuals based on their ethnic identification, hair texture, and skin tone.

In terms of MI about hair texture on IRO, EI, and GSE: AA women would be more affected by MI about hair texture than those who identified as Black. Hair typing 3a to $4 \mathrm{c}$, those that were tightly curled, would be more affected by MI about hair texture than those who identified as having straighter hair. And women who identified as having darker skin tones would be more affected by MI about hair texture than those who identified as having lighter skin.

\section{Importance of the Study}

Issues specific to Black females are ignored or go unnoticed. Specifically, hair texture, an aspect of AA and Black women's female identity, is often disregarded. To date, no existing study could be found that has examined MI about hair texture on IRO, EI, and GSE. As such, a theoretical model for the impact of MI about hair texture on IRO, EI, and GSE is lacking. This hinders further research, the development of awareness and prevention educational programs, and various needed interventions to combat the MI

about hair texture phenomenon that predominantly and negatively impacts Black women. 
More than a century after Emancipation, colorism, as it relates to skin color and specifically in this study, hair texture, continues to aggressively impact Black American life. Affecting everything from life expectancy and mate selection (Hughes \& Hertel, 1990; Hunter, 1998) to prison sentencing (Hochschild, 2007) and identity (Brown, 1998; Thompson \& Keith, 2004), colorism translates into real socio-economic differences (Hill, 1999) among both dark and light skinned Blacks (Herring, 2004). However, due to a continuum of preference not all Blacks are disadvantaged equally (Harrison \& Thomas, 2009). Most studies of race may marginally address the influence of skin color, but completely ignore the influence of hair texture variation on Black identity and racial experiences (Trevino, Harris \& Wallace, 2008). It is important to understand that hair texture discrimination does take place, is a real experience of Black people, and varies depending on one's position along both the skin color and hair texture continuum.

This research is essential because little if any research has been based on the impact of the media portrayals of Caucasian American women on AA women as it relates to hair messages, hair beauty, and its influence; and yet there is evidence to suggest that those who navigate the world who are classified as "beautiful" enjoy copious amounts of privilege. Those privileges range from academic success, job success, and in general more access that allows one entry to more and better resources to meet a desired end and ultimately achieve and succeed in unlimited areas of life. Hair is a dominant beauty aesthetic that is often devalued on the heads of AA women in its natural state. "Our culture teaches us that beauty matters more for women than almost anything else. The pursuit of beauty is a capital investment for women and the pursuit of white beauty, the 
only beauty there is in the United Sates, is doubly so for women of color" (Hunter, 2013, p. 63).

In addition this research attempts to discover relationships between variables that have been shown in previous research to be important but consequently have not been looked at together. Furthermore, these variables have been studied in part but AA women continue to be an invisible and overlooked group that goes understudied. This study brings out the importance of how MI about hair texture may be a contributing factor to IRO, affect AA women's sense of EI, and self-efficacy. Also, existing published research is limited or non-existent which calls for more research to better serve the AA and Black community in counseling, academia, personal growth, and overall societal awareness and change efforts.

\section{Definition of Terms}

Media: The main means of mass communication (broadcast, publishing, and the Internet) used to transmit information in American society (Allen, 2001). Media influences span various sources such as television, magazines, and Internet sources including Facebook, Instagram, Pinterest, Tumblr, and YouTube.

Hair texture: How hair, collective strands that grows from the head of humans, looks and feels. All hair has some type of texture and is classified in various different ways. Hair is classified as being "fine", "medium", or "coarse". These classifications largely refer thinness or thicknesses of hair strands, feel, and curl pattern. Hair patterns can be wavy, straight, curly, or coily. 
Internalized racial oppression: The adoption of racist messages by ethnic minorities that result in self-hatred and hatred toward their respective racial and ethnic group (Hall, 1986; Pyke, 2010).

Ethnic identity: Thoughts and feelings about one's ethnic group membership and its importance to the self (Yap et al., 2014), including a sense of belonging and behaviors toward one's ethnic group.

Self-efficacy: Also referred to as personal efficacy, is confidence in one's own ability to achieve intended results (Ormrod, 2006).

\section{Assumptions}

It is assumed that responses to the variables self-reported by the participants are reflective of their true feelings, knowledge, and experiences. It is also assumed that the instruments being used to collect data in this study - the Sociocultural Attitudes Toward Appearances Questionnaire, the Internalized Racial Oppression Scale (IROS), the Multigroup Ethnic Identity Measure (MEIM), and the GSE Scale - are reliable and validated.

I understand that I entered into this study with limited evidence and theory that was more based on my lived experiences and the real life accounts of many AA and Black women I've encountered or read about as it pertains to issues of hair texture. With this in mind, I still remained open to finding whatever the data revealed. To this end, I was committed to presenting the data set forth and open to any modifications of my original model to best fit the data. 


\section{General Methodology}

This study employed a nonexperiemental research design. The data was gathered using survey research. Survey research was appropriate for my study since the variables of interest were subjective. The sample for this study consisted of women who identified as AA or Black, 18 years or older, and who were raised in the United States. The sample was collected using convenience sampling. The sample was draw from respondents who were signed up to complete surveys on QuestionPro, an online service that helps researchers create and distribute surveys to targeted populations; a small literal arts university's student population in Southwest Michigan, through email invitation to participate in research given one met the survey demographic requirements; and social media platforms via a survey link invitation provided one met the survey demographic requirements.

Participant self-reports quantified all variables. Demographic variables were selfselected on multiple-choice items. Media influence, IRO, EI, and GSE were quantified through self-report on Likert scale items.

My data was analyzed using structural equation modeling (SEM) in order to determine the strength of the relationship between the variables. Structural equation modeling was a good fit for my study as it allowed me to examine my hypothesized model based on theory with actual data, which became the basis of my empirical model. Structural equation modeling also allowed for the analysis of both observed and unobserved (latent) variables. A partial confirmatory and model-generating approach was used as the respecified model was generated based upon the modification indices recommendations based on the data. The goals in this case, was to find the model that 
was both theoretically meaningful and well fitted to the data; and then use this model to determine if any within group differences existed pertaining to ethnic identification, hair texture, and skin tone.

\section{Limitations}

Several limitations are present in this study to be considered. Limitations are those characteristics within the research design that may impact or influence the application, interpretation, generalizability and utility of findings (Price \& Murnan, 2004). First, this study is intended to be informative about how the media impacts and influences AA and Black women's feelings about their hair texture and thereby affecting their EI, GSE, and IRO; however, the current study was nonexperimental in design. This means that there can be no direct cause-and-effect statements regarding statistical results. However, for this current study, a nonexperimental design was appropriate as I sought to measure variables as the respondents' experienced them. The model tested was based on the literature review outlined in Chapter 2. The literature detailed was a historic and experiential review of AA history and the Black experience captured in part as it pertains to issues of hair texture and beauty.

Second, all variables were assessed using self-reported measures. In addition, participants were made aware of what the study was aiming to evaluate and may have therefore resulted in participants answering questions in a socially desirable way versus in a manner that reflected their true beliefs, feelings, knowledge, or experiences. However, the nature of the variables made self-reported measures necessary in order to gather individualized subjective information. 
Third, my survey was conducted online, limiting my sample to individuals with Internet and computer assess, who were technologically savvy, and who had membership to QuestionPro, a small literal arts university in Southwest Michigan, or Facebook.

Lastly, I analyzed my data using SEM. Therefore, my results speak to the degree of fit observed between my resulting SEM model and the data from the current sample. As previously mentioned, it may be likely that different models (using different variables or even the same variables arranged in a different configuration) from different sample groups will render different results, meaning the model could be better fitted to the data or even poorly fitted. Structural equation modeling was a good approach for my research questions because it allowed me to assess multiple variables, both observed and unobserved, simultaneously.

\section{Delimitations}

Five delimitations are present in this study. First, is the use of convenience sampling. This research obtained results using QuesionPro, a survey database; a small literal arts university's student population in Southwest Michigan via email invitation; and social media, specifically Facebook sharing of the survey link. This implied computer literacy, Internet access, and group membership to QuestionPro, a small literal arts university in Southwest Michigan, or Facebook to obtain access. Second, the surveys were applicable only to respondents who were raised within the United States of America. Third, only individuals who identified as female, were 18 and over, and who identified as AA or Black were considered in this research. Age was due to consent, gender was due to focusing on an under-researched population group, and location was 
due to the study's focus. Fifth, the sample size was limited to a little over 300 participants due to financial and time constraints on the data collection process.

\section{Summary}

In this chapter, the background of the problem was outlined and the present study was introduced. The current study was designed to expand understanding of why a full range of adequate representation matters when it comes to MI about hair texture and related effects on AA women. Specifically, the contributions of IRO, EI, and GSE were examined to determine if they are related to the media's influence about hair texture on AA women. Additionally this study examined the within group relationships of MI about hair texture and IRO, EI, and GSE on ethnic identification, hair texture, and skin tone. The lack of current knowledge or ignoring of the issues of hair texture needs an empirical platform to validate on record the relational effects of MI about hair texture on IRO, EI, and GSE as they are related to various negative outcomes for AA women and reduced positive outcomes without awareness to combat and address the pervasive and longstanding issue at hand.

The hypothesis and research questions were defined in this chapter. The conceptual framework and assumptions of the study were also presented. Limitations and delimitations were discussed and the key terms, specific to this present study, defined.

This dissertation includes four more chapters. Chapter 2 introduces the reader to the existing body of literature on the subject matter and further explains the nature of the study. The theoretical framework of the Agenda-Setting Theory is discussed and the importance and significance for this study are explored in detail. Both formal and 
informal sources pertaining to understanding the issues of AA women and hair texture are presented.

Chapter 3 presents a comprehensive look at the methodology used in the research. It outlines the procedures used to obtain participants, data measures, and analysis of the data. Chapter 4 presents the results of the research. It describes the sample and variables. It also describes reliability of the instruments and correlations between the variables are explained. Finally, it presents the results of the model testing and modifications made to the hypothesized model as well as the tested within group models.

Chapter 5 discusses the implications and meaning of the study results, how the results pertain to the research, and presents a summary of the major findings. The work closes with recommendations for practice and future research. 


\section{CHAPTER 2}

\section{LITERATURE REVIEW}

\section{Introduction}

Many AA women (descendants of African slaves, also often interchanged with the word "black" or "blacks") spend large amounts of money on cosmetics and hair. Money spent on their hair is typically to alter it from its natural state to one commiserate with European standards of beauty. As a direct result of slavery, many AAs have been socialized to believe that to be attractive means possessing Caucasoid features (Woodson, 2002). The effects of media messages and their influence have continued to keep AA women in chains by the portrayal of European women coined as the beauty standard and the absence of unaltered AA women in media images. And a group simply cannot walk away from such systematic assaults unscathed, particularity if the assault continues in new ways, which is the modern day struggle (Wyatt, 1977). Just as skin color is classified and caste, AA hair texture is graded and given a value ranging from good to bad. Hair texture valuations are used alongside race markers, as hair is an epic way in which Black females are devalued (Greene, White, \& Whitten, 2000). Robinson (2011) notes that there is an intersection between hair as race that is particular to Blacks, and hair as beauty that is particular to females, which places a burden on Black females that is not experienced by either black males or white females. 
Hair is second only to skin color as a racial identifier in the United States; and is both historically and ancestrally tied to racial categorization (Arogundade, 2000; BoydFranklin, 1991; Mercer, 1990). Since tightly coiled, kinky, and afro hair textures are common only among African people (Banks, 2000; Spellers, 2003), racially motivated devaluations of African hair textures primarily affect those who have such hair texturesBlacks. These devaluations of African hair textures though the constant and influential messages from media and society are damaging to the AA psyche. The notion that black beauty and black women are not good enough as they are in turn translates into messages against black intelligence.

\section{Theoretical Framework}

The Agenda-setting theory serves as the theoretical framework for this study. The Agenda-setting theory, also known as The Agenda Setting Function of the Mass Media, which was first put forth by McCombs and Shaw (1972), describes the ability of media to influence the importance of topics to its viewers. This theory holds that the mass media has the powerful ability to bring issues to the attention of the public. That is, if a news or particular commercial item is covered frequently and prominently, the audience will regard the issue as more important. The basic claim is that as the media gives more attention to an issue, the public perceives the issue as important. The media sets the agenda, by implanting ideas into the minds of the general public, and the consumer viewer via consumption are influenced based on the messages propagated. In this case, the media constantly portrays hair textures that are either long, silky, straight, or smooth-European—which most, if not all ethnicities have deemed "important", "valuable", "attractive", "good", or to some extent better looking, more acceptable, more 
beautiful, and of great value. In the United States racial hierarchies celebrate one standard of beauty; and that beauty standard devalues and excludes most African hair textures, forcing AA females to fit into Eurocentric beauty paradigms that ultimately work against them (Arogundade, 2000; Gaskins, 1997; Taylor, 1999).

\section{African American Hair History}

Before the Civil War, the degree of pigmentation (skin color) meant the difference between living free or enslaved (Russell, Wilson, \& Hall, 1992). The preferential treatment of mulattoes by Whites laid the groundwork for a pattern of color classism in America that seeped deep into Black America (Russell et al., 1992). Post slavery, the "paper bag" and the "hair comb" test reinforced negative ideas about hair texture and skin color by offering preferential treatment, privilege, and benefits based on these physical characteristics (Bond \& Cash, 1992; Neal \& Wilson, 1989). Preferential treatment given by both Black and White cultures to AAs with light skin and other Caucasoid attributes, such as hair texture, has conveyed to many Blacks that the more physically conformed to the European standard of beauty, the more rewarding their lives would be (Bond \& Cash, 1992; Gatewood, 1988). In the early 1990's, there were often advertisements that suggested this would be the case. One advertisement noted by Rooks (1996) read,

Lighter Your Dark Skin: Race men and women protect your future... Be attractive. Throw off the chains that have held you back from prosperity and happiness that rightly belong to you. Apply Black and White Ointment (for white or colored folks) as directed on the package... be the envy of everybody (p. 30).

For AAs, skin color functioned as a marker of racial identity; therefore, products were marketed to offer AAs the opportunity to try to lessen the shame of such a marker 
(Rooks, 1996). Hair texture, just as it did many years ago, also functions as a marker of racial identity. Beauty manufacturers continue to target skin color and hair texture, a longstanding point of issue - the two characteristics AAs had to change if they expected to fit into majority culture American society (Rooks, 1996). As a result of such targeting, AAs have considered fair skin, White facial features, and White hair type to be more attractive than dark skin, Black features, and Black hair textures (Wade, 1996). In recent shifts, there has been the fawning over dark skin women, who are in the modeling world, as exotic, different, and something to be looked at, wow-ed over, and loved; however this recent drift, while currently trending, doesn't show in media acceptance or portrayal. It is an enigma of a very small sect of the modeling world and has shown to have limited media acceptance that doesn't represent the mainstream of majority culture.

Beauty is power and many women have gained success, socially and otherwise, banking on the marketability of their perceived physical attractiveness (Lakoff \& Scherr, 1984; Robinson \& Ward, 1995). Light skinned AAs still fare better economically, vocationally, and educationally (Wade, 1996). Values about skin color and hair texture get conveyed to children as they come out of the womb. Russell et al. (1992), notes that many Black families, following routine inquires about the baby's sex, weight, and health want to know "what's he or she look like?"-A veiled request and subtle, or not so subtle way for some, to ask about skin color and features of the child, which are then given some sort of value. In addition, similar to hair, comments about color, in relationship to skin, first come from family members and later from friends and peers, which then shape the psyche of a Black child as they grow from infancy to adulthood (Russell et al., 1992). Black children quickly absorb the guilt, anger jealousy, and depression generated in their 
families from unresolved color complexes (Russell et al., 1992).

In an attempt to unmask the notion of "self-hatred relative to colorism", Chambers, Clark, Dantzler, \& Baldwin (1994), conducted a study in which they investigated the relationship between perceived attractiveness, facial features, and African self-consciousness. They found that subjects with high African selfconsciousness scores were more likely to prefer African facial features as compared to Euro-American facial features. Additionally, they used more positive adjectives in describing these African facial features. Wade (1996) sough to measure skin color in relation to self-esteem, and self-perceived physical, sexual, and global attractiveness. He hypothesized that fair-skinned females would have higher self-esteem and self-ratings of attractiveness than dark skinned females. Wade also hypothesized that dark-skinned males would have higher self-esteem and self-ratings of attractiveness than fair-skinned males. No significant differences between the males and females were found, but the results showed that dark-skinned males had elevated ratings. Wade attributed the differences to the fact that many prominent AA males (entertainers and athletes) have darker complexions. To explain the lack of differences found between the women in the study, it is necessary to examine levels of Afro-centricity or Black consciousness as well as examining the "color socialization" of the women. For example, levels of self-esteem relative to skin color and hair texture depend on the values the subject's family of origin, circle of friends, and the immediate environment have placed on skin color and hair texture (Russell et al, 1992). In both of these studies the subjects were college students and alumni. With highly educated AAs, skin color, hair texture, and levels of attractiveness may serve different functions as compared to those AAs with less 
education and lower socio-economic status. African Americans who have lighter skin tend to identify less strongly with Black ethnicity, and more with middle-class white cultures and values (Hughes \& Hertel, 1990). Robinson and Ward (1995) measured the relationship between self-esteem and dating relationships. They found that there is a relationship between satisfaction with skin color and self-esteem. Their sample consisted of adolescents which gives weight to the idea that children are being socialized to attend to skin color, and other features related to aspects of beauty, such as hair texture, at a very young age. By the time these children become adults they already have a particular beauty standard imbedded within their psyche of which they need to conform, to fit in, or be accepted. This further speaks to the notion of how IRO may affect one's racial identity and self-esteem.

The "good hair, bad hair" distinction is the most deep-rooted construction of hair that occupies the psyche of AAs (Banks, 2000; Parks \& Woodson, 2002). Good hair is a phrase used within the AA community to describe an AA hair texture most symbolic with those belonging to non-black people (Eurocentric). In American culture, "good hair" is hair that is "manageable" with a texture on the softer and smoother side that is likely to be long, straight, wavy, or loosely curled. It is hair that minimizes African ancestry and reflects a European, Native American, or Asian ancestral mix within the AA individual. On the other hand, "bad hair" is hair that is deemed unmanageable, reflective of African ancestry, with and a texture on the harder side, tightly curled, tightly coiled, thick, and more likely to be short (Banks, 2000). It is hair described as "coarse," "unkempt," "nappy," or "kinky" (though use of the word "kinky" has been accepted by many within the AA and Black community as non-derogatory). 
Hair, unlike skin color, and to some extent facial features, is somewhat malleable and therefore easier to alter and control. As a result, AA women go through great lengths to make their hair beautiful and "appropriate" (Synnott, 1987). They will even sacrifice their physical well-being, risking obesity, avoiding exercise and certain activities, to maintain straight hair (Woolford, Woolford-Hunt, Sami, Blake, \& Williams, 2016). A study conducted by Woolford, et al. (2016) found that AA adolescent girls' preference for straight was a major contributor for avoiding exercise and activities that would cause them to sweat. Sweat jeopardizes hairstyles that require processing because sweat causes AA hair to "revert" back to its natural, tightly curled state.

As it relates to individuals, feelings and attitudes about hair texture, length, and style have many complex determinants. And these sensitivities and concerns may be symbolic of an AA's feelings about other aspects of themselves (Greene et al., 2000). These feelings are particularly intensified for AA women because the history of American racism has included an observable devaluation of African physical features and the establishment of beauty standards based on idealized depictions of white women's features (Greene et al., 2000; Hall, 1995; Martin, 1964; Neal \& Wilson, 1989). Although this discussion is focused on AA women, it should be noted that AA men also experience conflicts about their hair texture that can be damaging to their self-esteem (Greene et al., 2000). This research is focused on women, as physical appearance and attractiveness are more salient social variables for women than for men; and is often seen as the major determinant in calculating a woman's social value or status. Women experience greater overt distress and unconscious conflict about their hair and its relationship to their physical attractiveness than men (Green et al., 2000). Feelings about hair texture and 
styles also reflect intergenerational attitudes and conflicts within AA families (Greene et al., 2000). For example, a mother, who experienced conflicts about her own hair while growing up, may consciously or unconsciously, transmit these feelings to her daughter (Greene et al., 2000). There are many stories of AA women who recount in childhood the afternoon hair styling rituals in preparation for church the next day, or even school, and are characterized as torturous and extremely anxiety provoking. Green et al. (2000) and Grier and Cobbs (1980) describe the difficult task of grooming "black" girls' hair and note that this intensive grooming still does not transform the child into a beauty by the dominant cultures' standards, but is required just to make her "acceptable" or "minimally presentable". Furthermore, they suggest that many AA girls reach the subconscious conclusion that if their mothers have to inflict such pain on them to bring them just to the level of "acceptability", then they must have been ugly and "unacceptable" before the hair styling (Greene et al., 2000).

Because hair texture is tied into an AA's self-esteem and self-image, it is important to examine the historical significance of hair texture. Prior to the institution of slavery, hair had a very different meaning and purpose for Africans. Ever since African civilizations bloomed, hairstyles have been used to indicate a person's marital status, geographic location, age, religion, EI, wealth, and rank within the community. In some cultures a person's surname could be ascertained simply by examining the hair because each clan had its own unique hairstyle (Byrd \& Tharps, 2001).

\section{Hair Texture Consciousness Among African Americans}

Slave owners created distinct differences between enslaved Africans based on their skin color as well as their hair texture. Adjectives used to describe natural African 
hair include "bad", "nappy", "coarse", "unkempt", and or "wild", thus implying that it needs to be "fixed" or "controlled". These words reflect shame, embarrassment, and insecurity, therefore compelling many Africans, AAs, and Blacks to try to alter it. Curly and kinky hair was glorified in West African societies. When it comes to Black women's hair, Spellers (2003) noted, only kinky hair can be "twisted, locked, braided, weaved, curled, dyed, fried, and laid to the side" (p. 235). However, over time it became a symbol of inferiority and inadequacy once enslaved Africans reached the shores of America. The pride and elegance that once symbolized curly and kinky hair shifted and became a badge of ugliness and inferiority (Banks, 2000). Black hair is simultaneously the most versatile and the most devalued. Centuries of racism have instilled white beauty as the standard, compelling Black females to compare their own natural hair texture within the framework of white beauty paradigms — straight hair and long hair.

The combination of African, European, and Native American ancestral mixing was glorified and continues to be desired and highly sought after today. It also resulted in a myriad of skin tones and unique hair textures among descendants of African slaves in the Americas (Badillo, 2001; Montalvo, 2004). So now the perceived most beautiful AAs are those who look least like AA people (Hunter, 2004). Words used to describe Africans and AAs who had hair textures similar to textures of the Caucasian slave owners were "good, "curly", "soft", "nice", or a "good grade of hair." These terms were used to make the enslaved Africans feel superior to their counterparts- those who did not have this particular hair type or texture — based on the preferential treatment they received (Woodson, 2002). The quest for straight hair was often a torturous obsession for the slaves. Africans would often use household products to straighten their hair instead of the 
palm oil they were formally accustomed to. They would use oil-based products and equipment to achieve certain styles, like bacon grease and butter to condition hair, make it shine, and prepare it for pressing. They also used cornmeal and kerosene as scalp cleaners and lye mixed with other products such as potatoes to straighten the hair (Byrd \& Tharps, 2001). The lye would often eat the skin right of a person's head, but this did not deter its use. Kambon (1992) noted the act of hair straightening as a form of selfhatred, and even characterized it as pathological. He stated, “...I am thoroughly convinced that there is a powerful pathological dimension to African's approach to so called hair 'grooming' under the Culturally Misoriented conditions of White supremacy domination" (p. 142). This dangerous method of hair straightening wasn't just about conforming to the prevailing fashions of the day. Straight hair was highly sought after because it translated into economic opportunity, social advantage, and beauty. And it continues to carry this meaning today for many AAs (Byrd \& Tharps, 2001).

"Hair Typing" is a term used today to categorize hair, but began centuries ago. Hair typing based on hair texture details ten different hair types on a scale of 1 to 4 with subsets that include a through c (Appendix C): 1, 2a, 2b, 2c, 3a, 3b, 3c, 4a, 4b, and 4c. On this scale type 1 hair is straight, type 2 hair is wavy, type 3 hair is curly, and type 4 hair is kinky curly coily (Ruddock, 2014). Type 1 straight hair is typically oily, resilient, and has no discernible curl pattern. Type 2 wavy has three subsets or categories: 2 a, very loose wave, almost straight; 2b, loose waves; 2 , wavy with a deep S shape. Type 1 and Type 2 hair texture typically describe European, Asian, and Native American hair. Type 3 curly hair also has three subsets or categories: 3a, curly defined hair with loose ringlets; $3 \mathrm{~b}$, deeper more defined S shape coils; and 3c, tight spirals or coils. Lastly is type 4 hair, also 
containing three subsets or categories: $4 \mathrm{a}$, a mixture of ringlets and kinks that curls when wet but may dry into less defined curls; $4 \mathrm{~b}$, very tightly coiled, that may take on a zigzag curl shape or curled kinks that are more distinct and curls may not be visible when dry; and $4 \mathrm{c}$, extremely tight coils that shrink very close to the scalp when dry and with curls that may not be visible when dry (Ruddock, 2014). Type 3 and Type 4 hair textures typically describe African, AA, and mix combinations of Black hair. Type 3 and Type 4 hair, without the "help" of mixed ancestry, was classified as "bad hair" with Type 4 hair being the most undesirable. Though there has been movement within the AA community to embrace Black hair in all its forms, there is still a value scale that holds certain hair textures and hair lengths in higher esteem than others. This valuing and devaluing system takes place both from within the AA community and from without.

From 1820 to 1905 hair "pressing” emerged as a popular urban style among AAs who were financially established. It was around this time, roughly 1905, Madame CJ Walker invented the pressing comb and it consequently ushered in the era of altering the texture of natural hair (K. Imarogbe, 2003). However, although AAs did what they could to attain mainstream hairstyles, for all their efforts, they were still unacceptable to EuroAmericans (Byrd \& Tharps, 2001). "The free Black populations sprinkled about in cities like Boston and Philadelphia where they want to wear the same fashion and hairstyles as their white contemporaries only to find themselves ridiculed and satirized in the press, in the theatres and on the streets" (p. 18). Black people themselves internalized the concept of good and bad hair and amongst themselves propagated the belief that darker-skinned Blacks with kinkier hair were less attractive, less intelligent, and worth less than their lighter - hued brothers and sisters (Byrd \& Tharps, 2001). Distinct groups emerged that 
were formed based on the texture of a person's hair and skin-tone. Among the lightskinned elite were "blue vein societies," which excluded Blacks whose veins, which appeared blue under certain skin tones, were not visible beneath their skin; "paper bag tests," which allotted entrance into Black churches and other organizations based on comparison of one's skin color to a light brown paper bag; and "comb tests," (Kerr, 2005). In some churches a fine-toothed comb was hung from the front door. Anyone wanting to join the church had to be able to pass the comb smoothly through his or her hair. Acceptance was reserved for Black men and women with naturally straight hair able to withstand a fine-toothed comb. If one's hair was too "kinky" membership was denied (Byrd \& Tharps, 2001; Kerr, 2005). This was known as the infamous "Comb test". This type of blatant discrimination not only occurred in churches and social organizations, but it also occurred in the admissions process in historically black institutions such as Howard (established in 1867), Hampton (1868), and Spelman (1881), which were founded to educate the "Black elite" (Byrd \& Tharps, 2001). Photographs of the early graduates of these schools depict AAs of a lighter skin tone or with hair textures that resembled a Caucasian ancestor, suggesting that these attributes were one of the unspoken requirements for entry (Byrd \& Tharps, 2001). These continual negative biases toward AAs and privileges toward mixed blacks perpetuated and continue to perpetrate the systematic epidemics of slavery. For many Blacks straightening the hair and lightening the skin to feel good about one's self and to be accepted by both Black and Whites continued to be an obsession. Advertisements for skin lighteners and hair straighteners marketed by white companies continue to suggest to AAs that they need to change their physical features to be afforded class mobility and social acceptance by the 
dominant culture (Rooks, 1996). To gain access to the American dream and social acceptance AAs found it imperative to attempt to make White people more comfortable with their very presence. Education and training made little difference if a person looked too "African", that being possessing kinky hair, wide noses, and full lips; because those characteristics translated to "ignorant", "uncivilized", and "infantile" (Byrd \& Tharps, 2001) and so AAs did what they could to emulate European standards of beauty, dress, and behavior (Russell et al., 1992). In the fight for social acceptance many AA leaders were in favor of the increased attention to grooming and appearance, however many also denounced hair straightening as a poor attempt to emulate Whites and equated hair straightening with self-hatred and shame (Byrd \& Tharps, 2001).

Throughout the early 1900's into the early 1960's, AA's continued to be consumed with straightening their hair despite the procedure's serious health risk (K. Imarogbe, 2003). The chemical altering process used sodium hydroxide (lye) as the main ingredient in many hair-straightening formulas. This was also an industrial chemical, found in drain cleaners, oven cleaners and soaps (Kenyatta \& Kenyatta, 1996). Due to its caustic effects on drainpipes, causing erosion, the Food and Drug Administration banned the use of more than $10 \%$ of sodium hydroxide in household drain cleaners (Ferrell, 1996; K. Imarogbe, 2003). These same hair straightening chemicals using sodium hydroxide as the base could cause first to third degree burns, blindness, hair breakage, dermatitis of the scalp, hair dryness, scalp irritation, and lung difficulty (Bonner, 1991; Ferrell, 1996; K. Imarogbe, 2003; Kenyatta \& Kenyatta). These threats to the health and various injuries that would occur did not stop AAs from using hair-straightening products (Byrd \& Tharps, 2001). 
In the mid-sixties, Black hair underwent its biggest change since the arrival of Africans in the United States. There was a shift in perception of hair from one of style to statement. Black society took on the belief that the ways in which Black people wore their hair said something about their political affiliations and ideologies. For some, hair symbolized a continued move towards integration and equality while for others it represented a growing cry for Black power and nationalism (Byrd \& Tharps, 2001). Many AAs began to wear Afros, a symbol of "freeing your mind", and use hair to signify connectedness to their ancestors and to other Africans throughout the diaspora (Byrd \& Tharps, 2001). Afros represented the first time that Whites could look at you and collectively think, “This person doesn't go along with my philosophy and doesn't care that I know" (Byrd \& Tharps, 2001, p. 57). Hair became one of the symbols of the Black Power movement and the revival of cultural pride that had been lost as a result of slavery and integration. After generations of trying to neutralize and camouflage distinctive African features and characteristics, Black people began to celebrate them (Woodson, 2002). Former panther leader, Assata Shakur, explained in her autobiography, "We had never been exposed to any other point of view or any other standard of beauty". It was then that AAs coined the phrase, "Black is beautiful", and James Brown wrote the song, "I'm Black and I'm proud". As a result generational conflicts ensued in Black families between Black parents and grandparents who did not approve of the new acceptance of AA beauty or hair and still saw their children's appearance as disgraceful (Byrd \& Tharps, 2001; Shakur, 1987). This was the first time in Black American history, that those who did not adopt natural hair were judged harshly and publicly by many of their peers for their decision to wear straight, "neat" hair. The sought after "good hair" of the 
recent past became a badge of shame (Byrd \& Tharps, 2001). Jill Nelson wrote, "Bad hair became good; good hair became a badge of miscegenation forbears, a difficult cross to bear" (Byrd \& Tharps, 2001, p. 59). Black women in record numbers began to wear their natural hair texture and experiment with creative styles, like cornrows, braids, and African inspired head-wraps (Byrd \& Tharps, 2001). African Americans who did not have hair textures that would allow them to wear "Black" hairstyles went to great lengths to alter the natural textures of their hair to fit in (Byrd \& Tharps, 2001).

By the mid-seventies and into the eighties, many major Black leaders were dead, exiled, or in jail. Many of the brothers and sisters in the revolution had to get a job, and become more concerned about capitalism (Byrd \& Tharps, 2001). This meant a return to the hair straightening techniques of the past, as more conservative hairstyles were required in the workplace. The meaning of the Afro as it was known prior was no more. "African American women who had once stopped traffic with their gravity defying "fros were now as feathered, dyed, and silky smooth as their White peers" (Byrd \& Tharps, 2001, p. 104). During this time another chemical process was created, initially called the California Curl, and then the Jheri Curl. This "Black" version of the curly perm was very popular in the AA community. The Jheri Curl proposed to create a style that would require little maintenance and implied as a result, one would experience a carefree lifestyle. However the chemicals were harsh and left the hair severely dehydrated and therefore in constant need of lubricating oils, creams, and sprays (Byrd \& Tharps, 2001). Due to curl wearers being seen as being ashamed of their blackness and labeled sellouts, the Curl became increasingly less popular and soon became a taboo within the AA community (Byrd \& Tharps, 2001). 
The nineties ushered in a rebirth of cultural pride, and Black women again began to wear their hair in more natural and creative hairstyles. Myriads of women began to wear weaves, wigs, and add-on hair as never before. African Americans were finally able to afford hairstyles commiserate with that of Europeans without the torture of the past. In the late nineties, approximately 1.3 million pounds of human hair, valued at 28.6 million, was imported from countries (e.g. China, India, and Indonesia) where poor women sell their hair by the inch. Many AA women saw weaves as a way to achieve versatile looks and easily maintain a polished and acceptable appearance (Byrd \& Tharps, 2001). The long-standing problem of not having long, smooth hair was effectively solved for many. However, weave wearers were generally looked down upon and women who had naturally long tresses were most desirable. "The same men who would complement a woman on her long hair would look scornfully on the idea that it could be a weave" (Byrd \& Tharps, 2001). This fact alone recreated or uncovered the "good" vs. "bad" hair conflict that was dormant during the Black pride movement. It's as if the "decades of change" were just a brief departure from the ancient aesthetic status quo celebrating European features (Byrd \& Tharps, 2001). There was and still is a great desperation on the parts of AAs who do not possess hair textures like that of Whites. To be accepted was so important that they would go through the painful hair experiences to look "presentable", and suffer a great amount of distress, and financial burden to maintain it.

In her autobiography Assata Shakur (1987) describes one of her hair experiences in trying to look "presentable":

...I had always hated frying my hair-burnt ears, a smokey straightening comb, and the stink of your own hair burning. How many nights I spent trying to sleep on curlers, bound with scarves that cut into my head like a tourniquet... Afraid to go to the beach, afraid to walk in the rain, afraid to make passionate love on hot summer 
nights if I had to go to work in the morning. Afraid my hair would 'go back'. The permanent was even worse: trying to sit calmly while lye was eating its way into my brain...Clumps of hair falling out... (p. 174; as cited in K. Imarogbe, 2003).

This process became the standard in many AA homes. One that continues to set the stage for internalizing racial oppression and the idea that, the way one is born, is not good enough and the natural unprocessed hair that is a part of the African genetic code is not acceptable without altering. The fact that certain women did not have this experience because they have been born with physical characteristics that are compatible with the European aesthetics paved the way for and created an inevitable competition between the "haves" and the have nots" among heterosexual AA women for the attention and appreciation of AA men. It also created anxiety for women regarding their suitability, social acceptability, self-esteem and worth as it relates to their hair texture. An example of this is recorded in Hairpeace about the critical and conscious nature surrounding hair texture and length: According to K. Imarogbe (2003), Cleange (1993) noted an example of how critical and conscious of hair texture and length men can be:

...I delivered the news (to stop straightening her hair) to my smooth-faced boyfriend, who looked at me in horror and withdrew his pre-medical school arm from its customary place on my shoulders and said a bit self-righteously, I thought even then, 'well you do what you want, but I'll never touch your hair again' (Cleange, 1993, p. 40; as cited in K. Imarogbe, 2003).

Another example of this was a letter written to Ebony Magazine:

I truly need help with this one. I am a 26-year old women and I've been with my 29year old boyfriend for three years. I deeply love him and we've been talking about marriage lately. Recently, I let my hair grow very long. Once during love making, my boyfriend said, I love your beautiful long hair. It's just like a white girl's. This comment shattered my heart, not to mention my whole world. I love being Black and I'm proud to be a Black woman. Since this incident, I've noticed how much he talks about his best friend's wife who is white. When I discussed his comment about my hair with him, he said I'm overreacting. I don't think I am. I can't help but wonder if he really wants a white woman. I've considered breaking up with him. I just can't seem to get that comment he made out of my mind. Please help! D.J. Detroit, Mich 
(Ebony, 1996, p. 64).

These examples shed light on the experiences of women as it relates to hair perceptions and experiences. It also shows how even AA men are affected and have been unable to “... escape the confusing conundrum of experiencing pressure from family, peer, and/or society to have hair textures and partners that result in privileges within the Black community and the dominant culture..." (Greene et al., 2000, p. 172). But, it is important to note that AAs who fall on either end of the color or hair texture continuum may experience IRO, low self-esteem, or dissatisfaction with their hair.

Decades later, even to date, we still see the same desire by AAs to be accepted by both Euro-America and Black America. The aesthetic beauty standard for AA women is Eurocentric: smooth long hair, a thin lean build (size 0-5), and to have medium to light skin. However, there are times when the status quo is not the most desirable, and characteristics that commiserate more with African features are more desirable such as: full lips, a voluptuous curvy bottom, and tanned skin. So one may only image the distress experienced by AA women who possess these sought after desired characteristics that are then in turn only viewed acceptable or beautiful when worn or acquired by White women. African American women have bought into the European aesthetic and IRO due to decades of generational and intergenerational systematic assaults associated with her genetic makeup. The AA women's trauma story sold to her, taught to her, and fed to her over and over again of not being good enough has been deeply internalized, engrained, and transmitted into the very fiber of her being since birth. The systematic socialization of making AA women believe their own traits are unsatisfactory and then having them culturally appropriated by White woman is a heinous and longstanding systematic assault 
on the psyche, worth, and self-esteem of the black woman. It is one in which she may never be able to walk away from unscathed (Wyatt, 1977), as it has become a part of her generational and intergenerational history, identity, and trauma story.

\section{Media Influence}

The mass media (Internet, television, newspaper, magazines, and radio) are one of the most influential transmitters of information in American society (Allen, 2001). Statistics show that 6.9 million gigabytes of media flow into individuals and households per year, translating into 33 gigabytes per consumer daily (Riggott, 2013). In 2008 Americans consumed various types of media for 1.3 trillion hours, and average of 11 hours per person daily. By 2012, the total media consumption had increased to 1.46 trillion hours, an average of 13.6 hours per person daily. By 2015 data indicated that Americans would consume media for 1.7 trillion hours, an average of 15.5 hours per person daily (Riggott, 2013). As it pertains to Facebook, one of the most widely used media platforms today, statistics indicate that Facebook is accessed 8 times per day, 6 minutes per login, and an average of 15 days per month (Khew, 2016). In a 2015 study, were 10,000 AA adults were surveyed, AAs were said to watch the most television of any group. Television time, not including other social media platforms, was estimated at 20 hours per month, about 60 more hours than the total viewing audience combined (Loechner, 2015). AAs are the largest consumers of media and the least represented in a Eurocentric American culture that propagates and bolsters white beauty, silky smooth hair textures, and light skin.

The media is a profound source of cultural teaching with an unmatched power to reach millions quickly and conveniently (White, 2008). It is a "dominant force of 
socialization" that influences the social, emotional, and conscious being of its viewers (Kellner, 1995). The power of the media to transmit popular ideology goes beyond the ability to inform and entertain. The media is a transmitter that has been used as a tool of oppression (White, 2008). The various popular media mediums of today (Facebook, YouTube, Instagram, Twitter, SnapChat, et cetera) house a bombardment of images selling, teaching, and pushing out advertisements about how one should look, feel, dress, live, and think. The consumer culture in which we live makes it difficult to truly locate the origins of our own values and assumptions. Our values and assumptions appear to be a part of our natural world but are inevitably shaped by the subtle messages we consume (White, 2008).

Visual images play an important role in shaping perception. Images are understood as a representation of how one experiences the world, which one attaches ideas and give meaning to (White, 2008). The media tells women what they should look like and who they should aspire to be; while at the same time often representing women in a stereotypical, biased, and discriminatory way. These messages directed at women are magnified for AA women due to the absence of images that actually look like them and reflect their cultural heritage (White, 2008). The lack of visibility of AA women and the stereotypical manner in which they are represented greatly limit one's conception of themselves and ideas about what it means to be beautiful and accepted (White, 2008). African American women who rarely experience the full range of images that truly represent them in diversity of skin tones, hair textures, shape and size will begin to think that who they are is not acceptable and definitely not what's "in" (White, 2008). When in fact AAs are represented, content analyses reveal that the majority of media depictions of 
AAs women (in Black-oriented media and mass media) are those with light skin, straight hair, and small noses and lips (Capodilupo, 2010).

America's traditional and accepted standard of physical beauty is Eurocentric. The standards of beauty are created by and based on White women and their idealized features: straight blonde hair, fair skin, and thin bodies (Collins, 2002; Freedman, 1988). "These mythical ideals, though most often unattainable and unrealistic, are assumed to be the most definitive model of what we should look like and admire" (White, 2008). These normative beauty constructs set forth by the majority have been detrimental to the minority, especially AA women (White, 2008). When AA women are constantly exposed to images that highlight the dominant culture's ideas of beauty attributes, they will conclude not only that being "beautiful" is a female's proper role in society (White, 2008), but that one must aspire to and attain the dominant cultures definition of beauty to be accepted in that society. Advertisements for skin lighteners and hair straighteners marketed by white companies suggests to black women that only through changing physical features will persons' of African descent be afforded privilege and class mobility within AA communities and social acceptance by the dominant culture (Rooks, 1996). In the early 1990's, there were often advertisements that suggested this would be the case. One advertisement noted by Rooks read:

Lighter Your Dark Skin: Race men and women protect your future...Be attractive. Throw off the chains that have held you back from prosperity and happiness that rightly belong to you. Apply Black and White Ointment (for white or colored folks) as directed on the package... be the envy of everybody (p. 30).

The beauty myth keeps AA women in a state of fantasy. "This fantasy," asserts Wolf (2002), "is made of mirrors; no law of nature supports it. It keeps us spending vast sums of money and looking distractedly around us, but its smoke and reflection interfere 
with our freedom to be ourselves" (p. 45). Beauty is an ideological construct that structures feminine aesthesis—-skin color, hair texture, and body shape — and significantly affects the lives of women (White, 2008). "What makes one thing beautiful and another less is our psychological attraction, to some quality in the former that is absent from the latter, combined with equally implicit cultural biases (Lakoff \& Scherr, 1984). Beauty is an elusive source of power. Women have to work incessantly on controlling and managing their bodies to approximate an ideal that is often impossible to achieve (White, 2008). In the United States, the Eurocentric beauty standard (whiteness and Western European features) (Lakoff \& Scherr; Wolf, 2002) is damaging to the AA women's selfesteem and produces unconscious IRO. Theoretical and empirical research on the effects of ideals of beauty, among women of color, suggests that women of color are in a double bind (Kaw, 1994; Lakoff \& Scherr). Like all women, they are constantly bombarded with the notion that beauty should be their primary goal. But, as racial minorities, they are made to feel inadequate by an Anglo-American dominated cultural milieu that has historically both excluded them and distorted images of them in such a way that they themselves have come to associate those features (via internalized racism) stereotypically identified with their race with negative personality and mental characteristics (Kaw, 1994). Beauty has become so normalized that it no longer seems social, political, or racial in nature but "our entire cultural ethos in the contemporary United States seethes of white beauty" (Hunter, 2013).

Results from several studies examining the issue of Media's affects suggest that media images do influence AA adolescent girls' behavior and self-perceptions. In an experimental study of seven through twelve-year-old children, Oliviera (2000) found that 
black children exposed to magazine images emphasizing physical beauty reported lower physical self-concept than those exposed to images with no emphasis on beauty. Botta (2000) found that Black and White girls did not significantly differ in how much they compared themselves to idealized television images. For both groups, the more they idealized television images, the more dissatisfied they were with their bodies. Makkar and Strube's (1995) study of Black women found that those who possessed a strong racial identity rated themselves as more physically attractive after exposure to magazine images of attractive White women than did Black women with lower racial identity. In their study of television and body image, Schooler, Monique Ward, Marriwether, and Caruthers (2004) found that the relationship between watching Black programs and healthier body image only existed for the Black women who had lower racial identity scores. There was no relationship between watching Black programs and body image for the Black women who had high racial identity scores. Doll and picture preference studies with AA children conducted by Clark and Clark $(1939,1940)$ indicated AA's preference for and identification with Caucasian features. These studies do not address hair texture specifically, as there aren't studies that have investigated this subject matter, but they are evidence of how EI, self-esteem, and IRO occur from instances of media based on beauty standards and features propagated constantly, hair texture included. Hair type, length, and texture are a known and well-represented aspect of physical beauty.

Media images influence AA women to assume that there is one "ideal" and standard of beauty to which they must subscribe. The majority of mass media reinforces racist conceptions by constructing historically distorted favorable images of Whites and negative images of people of color, in particular AAs (Allen, 2001). It further transmits 
and perpetuates the message that only the existence of certain people is valid and worthy of recognition (Myers, 1993). Hence why the media can be a major source of oppression for AAs. Because media mediums reflect societal values and beliefs they transmit a powerful message about certain members of society, such as AA women. Mainstream media informs us of each ethnic groups place on the totem pole of hierarchy within social structures and present 'societal attitudes' toward minority members (Fujioka, 2005). The negative depictions of AAs prevalent in mainstream media (Entman \& Rojecki, 2001) represent society's negative attitudes toward and perception of AAs. Furthermore, the presence of AAs is noticeability missing in mainstream media (Ward, 2004). African Americans in the media, as stated previously, are often negative and support widely held stereotypical beliefs (athletes and gangsters) (Berry, 1998). This is an important point as to how and why mass media can be oppressive. Studies consistently show that AA children and adults consume more media than whites and other groups of color (see Ward, 2004). One national survey found the mean hours of television watching for White youth was about three hours, compared to five hours for Black youth (Ward, 2004). The paradox that Blacks are underrepresented (then often negatively represented when they are present in media) and overrepresented in the viewing audience has warranted investigation as to the impact of media consumption on Black viewers' self-perceptions, especially self-esteem.

Empirical examinations of the effects of mainstream media on AA children and adult's self-concept and identity formation have yielded mixed findings (Ward, 2004). Both mainstream and Black media have been noted to serve as primary sources of information about the in-group through which AA concepts and identity are developed 
and negotiated (Allen, 2001). Using a sample of 311 AA adults, Fujioka (2005) found that greater exposure to majority television had a negative effect on Black autonomy, African self-consciousness, and self-esteem (global self-worth). Majority print media had a negative effect on self-esteem but a positive effect on African self-consciousness (defined in the study as measure of Afrocentric worldview). Degrading and stereotypical images of AAs portrayed by mainstream media lowers self-esteem. How do AA women make sense of media images which tell them how they should look but also routinely “symbolically annihilate" them (White, 2008)?

\section{Internalized Racial Oppression}

Internalized racial oppression, also internalized racism, is one of the most common features of racism and yet the least studied (Hall, 1986). It is the "individual inculcation of the racist stereotypes, values, images, and ideologies perpetuated by the White dominant society about one's racial group, leading to feelings of self-doubt, disgust, and disrespect for one's race and or oneself' (Pyke, 2010). In other words, it is the adoption of racist messages by ethnic minorities that result in self-hatred and hatred toward their respective racial and ethnic group. Hall defines it as the "subjection of the victims of racism to the mystifications of the very racist ideology which imprison and define them". In literature and scholarly discourses it is also referred to as "internalized White supremacy," “internalized whiteness," and "racial self-hatred” (Pyke, 2010). W.E.B. Du Bois (1989) described White domination effects on Black Americans as a “double consciousness" in which AAs are born into "a world which yields him no true self-consciousness, but only lets him see himself though the revelation of the other world." He further explained, "It is a peculiar sensation, this double-consciousness, this 
sense of always looking at one's self through the eyes of others, of measuring one's soul by the tape of a world that looks on in amused contempt and pity (Du Bois, 1989). Nearly three decades later, the words of W.E.B. Du Bois still ring true. In a society where racial prejudice thrives in institutions, communities, politics, and popular culture it is extremely difficult for ethnic minorities to avoid absorbing and internalizing racist messages that constantly bombard them on every media medium. In the United States, racial hierarchies celebrate a single standard of beauty — a standard that devalues and excludes African hair textures, forcing Black females to fit into Eurocentric beauty standards that only work against them (Arogundade, 2000; Gaskins, 1997; Taylor, 1999). Hair is an important beauty determinant for American females (Gottschall, 2008; Weitz, 2004), and the connection of hair to female beauty intersects with race and gender, placing a particular burden on Black females whose natural hair textures and lengths are low on the beauty continuum (Badillo, 2001; Patton, 2006; White, 2008). Good and bad hair perceptions and hair valuations based on texture or length illustrate Black females' everyday struggles to fit Black hair textures into white beauty standards (Robinson, 2011).

In order to develop effective methods of resistance against IRO, it is necessary to understand how oppression is internalized and reproduced (Pyke, 2010). Minorities suffering from internalized racism subscribe to the belief, constantly transmitted to them, that Whites are superior to people of color. Minorities suffering from internalized racism may despise their own physical characteristics such as facial features, skin color, or hair texture. Others may stereotype individuals from their own ethnic group and refuse to associate with them, while some may refuse to identify with their group at all and identify as white. Internalized skin tone bias in communities of color is often spoken about, but 
there is also the unspoken internalized hair texture bias in communities of color that captures the "...pain and trauma of individuals who are reckoning with their own internalized racism or their experiences with internalized racism of others in their racial group, such as family members" (Pyke, 2010, pp. 553-554). The pain and trauma individuals suffering from internalized racism undergo result in actions and expressions such as "cosmetic surgery, hair straightening, skin lightening, and similar means of creating a more White-like appearance" that only reproduces the oppression of Blacks (Pyke, 2010, p. 554).

Though an uncomfortable topic for many, internalized racism must be talked about in order to strategize against it (Pyke, 2010). This is “... a topic that despite being pivotal to understanding racial inequality has been understudied for far too long" (Pyke, 2010, p. 553). There is also the "tendency to misconstrue internalized oppression as a reflection of some problem of the oppressed" (Pyke, 2010, p. 553). It is not the result of a cultural or biological characteristic of the subjugated; nor is it due to any weakness, inferiority, psychological defect, gullibility, ignorance, or other shortcoming of the oppressed (Pyke, 2010). The internalization of oppression is a multidimensional phenomenon that takes on various forms and sizes across situational contexts and intersections of multiple systems of domination (Padilla, 2001; Pyke, 2010). It is an inevitable condition of all structures of oppression and cannot be reduced to just one form or type; or assumed to affect similarly located individuals or groups in precisely the same way (Pyke, 2010; Schwalbe et al., 2000). 


\section{Ethnic Identity}

Ethnic identity is defined as thoughts and feelings about one's ethnic group membership and its importance to the self (Yap et al., 2014), including a sense of belonging and behaviors toward one's ethnic group. Ethnicity and race are often used interchangeability, though some scholars argue the two are not synonymous terms. Ethnicity is defined as relating to large groups of people classed according to common racial, national, tribal, religious, linguistic, or cultural origin or background. Ethnicity is defined as a class or kind of people unified by shared interests, habits, or characteristics (Ethnicity, n.d.). Race is a defining construct in American society, but it is not the same as ethnicity (Hunter \& Sellers, 1998). According to Hunters \& Sellers, race is a marker for ethnicity and culture while being associated with structural inequality. Race carries with it a sense of shared history of a group, but also represents the distinction of structural inequality, injustice, and discrimination experienced by a group (Hunter \& Sellers, 1998). Ethnicity is used as a synonym for race, however, according to Helms (1994), it is a better descriptor for social identity as it is connected to the culture of a group an individual belongs to as well as that of his or her ancestral group (Helms, 1994).

Race is purported to be fluid, multidimensional, and experienced not only in accord with how one self-identifies but also with how one is perceived by others (Vargas \& Kingsbury, 2016). However, the vast majority of survey-based sociological research, as well as society, only employ one-dimensional measures of racial classification. Race and racial identity can be fluid and multidimensional, however the social, political and economic meanings of race have not been fluid due to an insistent rigidity in our society (Onwuachi-Willig, 2016). A person's personal and collective group member's 
experiences can clash with the ideology of social systems in attempting to categorize them. Meaning that individuals with their own personal biographies about the self will struggle to maintain that identity when society and social systems tend to dictate it (Taifel, 1982). Ethnic identity within society is often categorized as race. However race is not biological, it is a social construct (Onwuachi-Willig, 2016). It is for these reasons that the assessment of racial and EI often times overlap in the field (Helms, 1995; Sciarra \& Gushue, 2003).

Positive EI has been linked to aspects of positive adjustment, self-esteem, subjective well-being, and reduced depressive symptoms (Yap et al., 2014). Ethnic identity is considered a critical component of the self-concept and crucial to the psychological well-being of members of an ethnic group; however questions still remain about what EI is and how it should be measured (Roberts et al., 1999). One of the problems Phinney (1992) noted on measuring ethic identity is that only a few scales exist assessing ethnic or racial identity for multiple groups at a time. In addition ethnic conflict professors, such as Steve Saideman, and others have argued and noted the following challenges:

Ethnicity, like race, is a social construct, but it's still a construct with significant implications for the world. How people perceive ethnicity, both their own and that of others, can be tough to measure, particularly given that it's so subjective. So how do you study it (Fisher, 2013)?

Before Phinney established the MEIM, approaches to measuring ethnic or racial identity varied and served particular populations separately. However, the MEIM is not without its own challenges. Cross (1994) outlined his theory of Nigrescence, which explored blackness and AA identity. Nigrescence is a word of Latin origin that describes a process of becoming black or developing a racial identity. Cross' model of Black racial 
identity development, which was first detailed in a 1971 publication (his book Shades of Black published in 1991), relates the transition of Black identity through a five-stage acquisition to Black Identification - the process of becoming Black. The five stages include Pre-encounter, Encounter, Immersion/Emersion, Internalization, and Internalization-Commitment. The Cross Racial Identity Scale (Vandiver, Cross, Worrell, \& Fhagen-Smith, 2002) was developed after revision of Cross' Nigrescene stages. Phinney proposed a three stage model of EI development, developed to study teenage and adolescent populations, which included Unexamined EI, EI Search/Moratorium, and EI Achievement. In the first stage, individuals have a lack of interest in exploring their ethnic background. The second stage a harsh or indirect event precipitates development of a person's EI. In the third and final stage, the individual comes to terms with who they are and gains a clear sense of their EI. Helms (1990) also developed black and white racial identity scales focusing on five developmental stages, the Black Racial Identity Attitude Scale, being her more prominent work. Sellers, Smith, Shelton, Rowley, and Chavous (1998) introduced the Multidimensional Model of Racial Identity in order to understand the significance of race in the self-concepts of AAs. They maintain that although many ethnic groups have experienced discrimination and oppression, the experiences of AAs in the Unites States are significantly different.

Matters of race and ethnicity are doubly complex for Black people. "Racialized beauty standards combined with the color complex make hair texture and length an essential part of Black female identity" (Robinson, 2011, p. 4). In addition, Black people in the United States are particularly susceptible to mainstream or dominant cultural 
messages and influences that devalue physical features common to African people due to the lack of a strong collective African identity (Azibo, 1989).

\section{Self-Efficacy}

Self-efficacy, also referred to as personal efficacy, is defined as confidence in one's own ability to achieve intended results (Ormrod, 2006). Psychologist Albert Bandura defined self-efficacy as one's belief in their ability to succeed in specific situations or accomplish a task (Luszcynska \& Schwarzer, 2005). Psychologists have studied self-efficacy from many perspectives and paths of development or lack thereof. These aspects involve the contribution and interaction of self-efficacy to self-esteem, self-concept, and behavior. Self-efficacy affects every area of human endeavor and has the capacity to play a major role in how a person approaches goals, tasks, challenges, and life (Luszcynska \& Schwarzer, 2005). At the center of self-efficacy is Bandura's social cognitive theory, which emphasizes the role of observational learning, social experiences, and $\mathrm{MI}$ in the development of knowledge and personality. One of the main concepts of social cognitive theory is that an individual's actions, feelings, and responses are influenced by what that individual has observed in others. Self-efficacy is developed from external experiences and one's own self-perception. These self-perceptions are influenced, even persuaded, by personal and external social factors (Bandura, 1977, 1988; Miller \& Dollard, 1941; Mischel \& Shoda, 1995).

The propagated influences of media messages as well as social experiences over long decades which have discriminated against darker skin and hair classified as "bad" or "unkempt" have caused Black women to internalize the influential concept of good and bad hair amongst themselves and believe the messages broadcasted that darker-skinned 
Blacks with kinkier hair textures are less attractive, less intelligent, and worth less than their lighter hued counterparts (Byrd \& Tharps, 2001). Oscar-winning actress Lupita Nyong'o recently addressed this issue, when her hair was altered on images of the November 2017 Grazia magazine, and commented in response:

As I have made clear so often in the past with every fiber of my being, I embrace my natural heritage and despite having grown up thinking light skin and straight, silky hair were the standards of beauty, I now know that my dark skin and kinky, coily hair are beautiful too.... [T] here is still a very long way to go to combat the unconscious prejudice against black women's complexion, hair style and texture. \#dtmh (don't touch my hair) (Gibson, 2017).

This unconscious prejudice Nyong'o speaks of fills and permeates into every avenue of the AA woman's life as she tries to navigate a world that refuses to accept her as an equal and level the playing field of the beauty realm.

White beauty is normal beauty and white beauty is ideal beauty. Our culture teaches us that beauty matters more for women than almost anything else. The pursuit of beauty is a capital investment for women and the pursuit of white beauty, the only beauty there is in the United Sates, is doubly so for women of color (Hunter, 2013, p. $63)$.

The headlines are filled with messages that are anti-black beauty and hair discrimination as it relates to AA women.

In 2012, Rhonda Lee, a meteorologist anchor in Louisiana, was fired for defending her hair choice after a white male viewer commented on the news Facebook page that Lee needed to "wear a wig or grow some more hair," because he, a white male, didn't think her short natural hair looked good on TV. Lee responded:

I am very proud of my African American ancestry which includes my hair. For your edification: traditionally our hair doesn't grow downward. It grows upward. Many Black women use strong straightening agents in order to achieve a more European grade of hair and that is their choice. However in my case I don't find it necessary. I'm very proud of who I am and the standard of beauty I display. Women come in all shapes, sizes, nationalities, and levels of beauty. Showing little girls that being comfortable in the skin and HAIR God gave me is my contribution to society. Little 
girls (and boys for that matter) need to see that what you look like isn't a reason to not achieve their goals (Starr, 2014).

In 2013, 7-year-old Tiana Parker, and straight A student, was sent home from her school in Oklahoma because, as she stated, "They didn't like my dreads." The school's dress code, since amended, forbade Tiana's hairstyle citing that it was a "faddish hairstyle" (Andrews, 2014). Dreads, dreadlocks, and or locks (also locs) are a type of hairstyle many AA's wear, for stylishness or ethnic pride, as their hair easily coils together to create the style and maintain.

In 2016, Southern Sudan model Nayadak Duckie Thot, popularly known as Duckie, was bullied for being black (she has a night-shaded skin tone) and because of her hair. She is reported as having explained her experience by stating,

Being a black woman, we haven't really been taught how to take care of our natural hair - we've only been taught how to hide it. I think hair companies, the media, hairstylists, and the industry itself are to blame. They haven't made the same efforts to ensure black women are looked after in their most natural form (Allen, 2016).

The Army became famous for its slogan "Be All You Can Be," but Black females could only do that if their hair styles fit into white paradigms of what was considered neat as the military used terms such as "matted and unkempt" in their grooming guidelines (Rhodan, 2014), which directly applied to Black, not White hairstyles. It wasn't until recently that the military lifted its ban on "Black hairstyles" after months of backlash and court proceedings. Three branches of service including the Army, Air Force, and Navy after court review agreed to change their grooming regulations to include hairstyles that essentially were ethnically inclusive and sensitive to the needs of Black women. All three branches also agreed to remove the terms "matted and unkempt" from their guidelines. Rep. Marica Fudge (D-Ohio), chair of the Congressional Black Caucus who initiated 
proceedings against the military's decision to ban natural hairstyles such as dreadlocks and twisted stated,

These changes recognize that traditional hairstyles worn by women of color are often necessary to meet our unique needs, and acknowledges that these hairstyles do not result in or reflect less professionalism or commitment to the high standards required to serve within our Armed forces (Rhodan, 2014).

Hairstyle choice and hair straightening as a means of conforming to the majority beauty standards, for some, is also a means of survival. Many AA women are faced with the reality of choosing to wear their natural hair and risk their jobs and opportunities for advancement or fall in line and conform to the standards set forth by the majority that essential obliterate hair types and textures that are uniquely germane to AA and Black women. Kimmika Williams-Witherspoon, who chooses to wear her natural hair at a university where she has not yet gained tenure but has observed other AAs who didn't wear natural hairstyles until after they were tenured, stated,

In reality, how I dress and my hairstyle should not indicate to you my capacity to fulfill a job, but you have to make real conscious choices. We always will have to wear a mask. It hurts me to say that, but that seems to be the reality (Dossou, 2013).

These are just a few examples of the many stories and experiences AA women face pertaining to their appearance that society and institutions attempt to "white-wash" by ethnic obliteration that overtly affects their personal lives, sense of well-being, learning, education, career, and job statuses. Their ability to achieve and succeed is often limited and controlled by their willingness to comply with beauty standards cloaked as company appearance policy. Ursula Burns, former chairwoman and Chief Executive Officer of Xerox, current chairwoman of VEON, one of the largest integrated telecommunications services operators globally, wears a short natural crop cut and seems to have defied the odds. However, many black women face the harsh reality that "a black 
woman's hair choice can limit her mobility" (Andrews, 2014, p. 48). During an

interview, attorney Angela Reddock stated:

You develop a sense of what makes people feel more comfortable in corporate America. The reason many of us straighten our hair is to take hair out of the equation. Folks in the boardroom can actually hear what we're saying instead of being distracted by our hair.... Someone like CEO Ursula Burns, who wears her natural, is certainly a pioneer. But there are not many Ursula Burnses at the top of the business world (Andrews, 2014, p. 48).

This notion that AA women must take their hair, a physical trait that is deeply rooted in ethnicity, deoxyribonucleic acid, and cultural makeup, and erase it by conformity to the White beauty standard to make other Whites comfortable just for the opportunity to be heard, seen as an equal, or be granted the possibility of achieving in life has been the longstanding struggle of AA women for decades.

Historically, "good hair" has been considered long, straight, and similar in texture to the hair of White, European American women (Buchanan, 1993; R. Hall, 1995; Neal \& Wilson, 1989). In fact, long, straight hair has been idealized and associated with higher social status in the AA community (Greene et al., 2000). On the other hand, hair that is "kinky," "nappy," and short is characterized as "bad" (Buchanan, 1993) and synonymous with darker skin, lack of intelligence, and all accompanying negative connotations (R. Hall, 1995). It has been shown that (similar to skin tone) women on either end of the hair continuum (long and straight, to short and kinky) receive extreme reactions-idealization to invalidation (Green et al., 2000). For women who possess these features, there is a risk or feeling unattractive or shameful (West, 1995). Reactions to these derogatory images of AA women with these beauty features are often raised in therapy because they are so salient and powerful (West, 1995). It has even been suggested that how a woman feels about her hair may be representative of how she feels about herself (Greene et al., 2000). 
What then does she feel about what she can achieve and accomplish in life? This damage to self that says black beauty must be corrected to the mold of white beauty extends into black intelligence. "Our country has a long history of portraying African Americans an intellectually inferior, which is particularly likely to affect their participation in a field that focuses so single-mindedly on the quality of one's intellect" (Andrews, 2014, p.48). The link between perceived physical beauty and personal self-efficacy is unknown; hence the need for this study, however there is evidence to suggest that those who navigate the world who are classified as "beautiful” enjoy copious amounts of privilege. Those privileges range from academic success, job success, and in general more access that allows one entry to more and better resources to meet a desired end and ultimately achieve and succeed in unlimited areas of life.

\section{Summary}

Representation matters. The lack of visibility of AA women and the stereotypical manner in which they are represented greatly limit one's conception of themselves and ideas about what it means to be beautiful and accepted (White, 2008). African American women who rarely experience the full range of images that truly represent them in diversity of skin tones, hair textures, shape and size will begin to think that who they are is not acceptable and definitely not what's "in" (White, 2008). These normative beauty constructs set forth by the majority have been detrimental to the minority, especially AA women (White, 2008).

In the United States, racial hierarchies celebrate a single standard of beauty, a standard that devalues and excludes African hair textures (Arogundade, 2000; Gaskins, 1997; Taylor, 1999). Beauty has become so normalized that it no longer seems social, 
political, or racial in nature but "our entire cultural ethos in the contemporary United States seethes of white beauty" (Hunter, 2013, p. 63). Media images influence AA women to assume that there is one "ideal" and standard of beauty to which they must subscribe. The majority of mass media reinforces racist conceptions by constructing historically distorted favorable images of Whites and negative images of people of color, in particular AAs (Allen, 2001). As a result of America's history of slavery and the effects of MI that contribute to continued oppression, EI and self-efficacy within the AA community is lost. The extent of the media's influence about hair texture as it relates to AA women's ethnicity, self-efficacy, and IRO is not known due to a lack of investigation on the topic. There is an absence of information on the specific role of MI and hair texture, and there is even less information on how MI about hair texture impacts IRO and self-efficacy of AA women. Little if any research has been based on the impact of the media portrayals of Caucasian American women on AA women as it relates to hair texture messages. Meanwhile, more negative images of American women than Caucasian American women continue to appear in the media (Frisby, 2004; Hill, 1999; Thomas, 2006). African American women are bombarded with negative images of themselves (Allen, 2001; Woodard \& Mastin, 2005) while being presented with an unattainable beauty standard that has gone unquestioned or challenged for decades. As a result, trauma, internalized racism, and low self-esteem trickles into all avenues of AA life unbeknownst to the AA and Black community.

This damage to self that says Black beauty must be corrected to the mold of White beauty extends into black intelligence. "Our country has a long history of portraying AAs an intellectually inferior, which is particularly likely to affect their 
participation in a field that focuses so single-mindedly on the quality of one's intellect" (Andrews, 2014, p. 49). The link between perceived physical beauty as it relates to the hair texture of AA women and personal self-efficacy is unknown; hence the need for this study, however there is evidence to suggest that those who navigate the world who are classified as "beautiful" enjoy copious amounts of privilege. Those privileges range from academic success, job success, and in general more access that allows one entry to more and better resources to meet a desired end and ultimately achieve and succeed in unlimited areas of life.

Eurocentric features result in more access, a less threatening presentation to White America, and is a means of survival for many who would normally due to stereotypes and biases be seen as unattractive and unlikable. Centuries of racism dictate white beauty standards, compelling Black females to rate their own natural hair texture within the framework of straight hair paradigms. "Racially motivated, concepts of good and bad hair are bad for Black females because they devalue hair textures most Black females have" (Robinson, 2011, p. 6). The propagated influences of media messages as well as social experiences over long decades which have discriminated against darker skin and hair classified as bad have caused Black people to internalized the influential concept of good and bad hair amongst themselves and believe the messages broadcasted that darkerskinned Blacks with kinkier hair are less attractive, less intelligent, and worth less than their lighter hued counterparts (Byrd \& Tharps, 2001). The extent of this impact is the focus of this research. 


\section{CHAPTER 3}

\section{METHODOLOGY}

\section{Introduction}

In this chapter, I will describe the research design used to examine the impact of MI about hair texture on IRO, EI, and GSE within the general population of AA females within the United States. This study used survey research as part of a nonexperiemental research design. The current study used a multivariate, correlational research design. Participants completed a demographic questionnaire and surveys measuring (a) MI about hair, (b) IRO, (c) EI, and (d) GSE. Structural equation modeling was used to test the proposed model of the relationship between participants' level of IRO, EI, and level of GSE based upon MIs about hair texture. The fitted model was then used to determine if there were between group differences related to ethnic identification, hair texture, and skin tone.

\section{Research Design}

This study employed a nonexperimental, quantitative, correlational, crosssectional, survey research design. Nonexperimental describes research in which the researcher does not control, manipulate, or modify the predictor variables (IRO, EI, and GSE) in any way. Instead, the researcher relies upon data interpretation and observations on which to draw conclusions. A nonexperimental design was appropriate for my study in 
order to measure the relationship between MI about hair texture, IRO, EI, and GSE based upon the respondent's level of influence, feelings, and experiences.

Quantitative methodology was the most fitting for this current research, as it allowed for exploration of the relationship between the variables and testing of the hypothesis using structured statistical measures. Quantitative research is defined as explaining a phenomenon by collecting numerical data that are analyzed using mathematically based methods, in particular statistics (Aliaga \& Gunderson, 2000). A quantitative research design entails the collection of numerical data for the purpose of exhibiting the view of relationships between theories and research as deductive, demonstrating an objective conception of social reality (Bryman, Teevan, and Bell, 2005).

This study was correlational because it attempted to find relations among variables (MI, IRO, EI, and GSE). The correlational method involves looking at relationships between two or more variables, neither of which are under the control of the researcher (Salkind, 2011). Correlations can be strong or weak, as well as positive or negative. In some cases, there may be no correlation at all between the variables of interest. While correlational research can reveal if a relationship exists between variables, this kind of research cannot prove that changes to one variable lead to changes to another variable; correlational studies cannot prove cause-and-effect relationships (Salkind, 2011). Therefore to determine the correlation between the above-mentioned variables a correlation coefficient will be used. The Pearson correlation coefficient $\left(r^{2}\right)$ examines the extent of the relationship between variables (Salkind, 2011). 
This study was cross-sectional because it sought to both examine and compare different groups of individuals at a specific point in time. Pertaining to the survey method of gathering data, survey research allowed me to select a sample of participants from a population and administer a standardized questionnaire to them. To determine the correlational relationship between the variables being studied, data was collected from separate scales administered to study participants, which will be discussed in the instrumentation section below.

\section{Population and Sample}

The sample for this study consisted of adult females, 18 years and older, who were raised in the United States of America, and identified as AA or Black. The sample was collected using convenience sampling. Participants were drafted via QuestionPro, an online service that helps researchers create and distribute surveys to targeted populations; the student population of a small liberal arts university in Southwest Michigan via email invitation; and social media, specifically Facebook. QuestionPro provides participants with points in exchange for completed surveys that may be used to redeem various rewards such as gift certificates to use at a later time. Both the student population of a small liberal arts university in Southwest Michigan and individuals via social media where invited to participate in the current research study if they fit the targeted population. No compensation was provided. The total sample population consisted of 322 participants. 


\section{Hypothesis and Research Questions}

The main hypothesis of this study was that the reproduced covariance matrix proposed in the theoretical model (see Figure 1) and the observed sample covariance matrices were equal. This hypothesis addressed the following research question: is the hypothesized theoretical model a good fit to the sample? As depicted, the theoretical model proposed a direct effect between MI and IRO, between MI and EI, and between MI and GSE.

From the results of the main hypothesis, this study then sought to address the following research question: does ethnic identification, hair texture, or skin tone influence the fit of the empirical model? That is to say, does the fitted model relationship between MI and IRO, between MI and EI, and between MI and GSE affect individuals differently depending on ethnicity, hair texture, or skin tone? And if so, based on the structural model, to what extent?

\section{Definitions of Variables}

\section{Exogenous Variables}

In this study, one exogenous (independent) variable was measured: MI about hair texture. This section and the next section will describe the conceptual definition of each variable and the instruments that were used to measure each variable. The psychometric properties of the scales and the specifics of the items used are described in the Instrumentation section.

Media, in this research, was defined as the main means of mass communication used to transmit information and exert influence (Allen, 2001). Media influences span various sources including television, magazines, and internet sources such as Facebook, 
Twitter, Instagram, Pinterest, Tumblr, and YouTube, to name a few. This variable was measured using the Sociocultural Attitudes Toward Appearances Questionnaire, $3^{\text {rd }}$ Edition (SATAQ-3) Modified. This instrument was slightly modified by the researcher, which will be discussed further in the Instrumentation section.

\section{Endogenous Variables}

The present study measured three endogenous (dependent) variables: IRO, EI, and GSE. Internalized racial oppression was defined as the adoption of racist messages by ethnic minorities that result in self-hatred and hatred toward their respective racial and ethnic group (Pyke, 2010). This variable was measured by The IROS. Ethnic identity was defined as thoughts and feelings about one's ethnic group membership and its importance to the self (Yap et al., 2014), including a sense of belonging and behaviors toward one's ethnic group. This variable was measured by the MEIM. Self-efficacy was defined as confidence in one's own ability to achieve (Ormrod, 2006). This variable was measured by the GSE.

Table 1 lists the conceptual definitions of the variables included in my study. It also depicts the survey items used to measure each variable. For variables drawn from instruments with multiple subscales, this will give the reader a general idea of what each subscale represents.

\section{Instrumentation}

Instruments for this study consisted of four surveys and two questionnaires: The SATAQ-3 Modified (by the researcher of this study), The IROS, The MIEM, The GSE, and a demographics and hair texture questionnaire to assess age, ethnicity, place of birth, 
Table 1

Conceptual Definitions of Variables and Sources of Associated Survey Items

\begin{tabular}{|c|c|c|}
\hline $\begin{array}{l}\text { Variable name } \\
\& \text { Instrument(s) }\end{array}$ & Conceptual definition & $\begin{array}{l}\text { Instrument, subscale, \& } \\
\text { items used }\end{array}$ \\
\hline $\begin{array}{l}\text { Media Influence } \\
\text { about Hair Texture } \\
\text { (measured by } \\
\text { items from the } \\
\text { SATAQ3 } \\
\text { Modified }\end{array}$ & $\begin{array}{l}\text { The main means of mass } \\
\text { communication used to transmit } \\
\text { information and exert influence } \\
\text { (Allen, 2001). Media influences } \\
\text { span various sources including } \\
\text { TV, magazines, and internet } \\
\text { sources such as Facebook, } \\
\text { Instagram, Pinterest, Tumblr, and } \\
\text { YouTube, to name a few. }\end{array}$ & $\begin{array}{l}\text { SATAQ-3 Modified: } \\
\text { Information }(1,5,9,13,17, \\
20,23,26,27,29,) \\
\text { Pressure }(2,6,10,14,18, \\
21,24,30) \\
\text { Hair }(3,4,7,11,12,19,22) \\
\text { Appearance }(8,15,16,25, \\
28)\end{array}$ \\
\hline $\begin{array}{l}\text { Internalized Racial } \\
\text { Oppression } \\
\text { (measured by } \\
\text { items from the } \\
\text { IROS) }\end{array}$ & $\begin{array}{l}\text { The main means of mass } \\
\text { communication used to transmit } \\
\text { information and exert influence } \\
\text { (Allen, 2001). Media influences } \\
\text { span various sources including } \\
\text { TV, magazines, and internet } \\
\text { sources such as Facebook, } \\
\text { Instagram, Pinterest, Tumblr, and } \\
\text { YouTube, to name a few. }\end{array}$ & $\begin{array}{l}\text { IROS: } \\
\text { INS }(4,7,18,22,24,26,27) \\
B R H(2 \mathrm{R}, 6,8,11 \mathrm{R}, 13,15, \\
16 \mathrm{R}, 20) \\
A P A(1,3,12,14,17,19, \\
21,25) \\
H C(5,9 \mathrm{R}, 10,23,28 \mathrm{R})\end{array}$ \\
\hline $\begin{array}{l}\text { Ethnic Identity } \\
\text { (measured by } \\
\text { items from the } \\
\text { MEIM) }\end{array}$ & $\begin{array}{l}\text { Thoughts and feelings about one's } \\
\text { ethnic group membership and its } \\
\text { importance to the self (Yap, et. al, } \\
\text { 2014), including a sense of } \\
\text { belonging and behaviors toward } \\
\text { one's ethnic group. }\end{array}$ & $\begin{array}{l}\text { MEIM: } \\
\text { EI Search }(1,2,4,8,10) \\
\text { EI } A B C(3,5,6,7,9,11,12)\end{array}$ \\
\hline $\begin{array}{l}\text { Self-Efficacy } \\
\text { (measured by } \\
\text { items from the } \\
\text { GSE) }\end{array}$ & $\begin{array}{l}\text { Confidence in one's own ability to } \\
\text { achieve (Ormrod, 2006). }\end{array}$ & All GSE items, no subscales \\
\hline
\end{tabular}


where one grew up, identified hair texture, skin tone, and various feelings pertaining to one's hair texture.

\section{The Sociocultural Attitudes Towards Appearance Questionaire-3 Modified}

The SATAQ-3 is composed of 30 items intended to measure one's endorsement of societal appearance ideas (Thompson, van den Berg, Roehrig, Guarda, \& Heinberg, 2004). It has four subscales that assess multiple aspects of societal influence including two dimensions of internalization of appearance, pressure, and information. Participants are asked to read questions concerning appearance and then respond to them using a 5point Likert scale ranging from 1 (definitely disagree) to 5 (definitely agree). Example items for the SATAQ-3 Modified information subscale are "TV commercials are an important source of information about hair and being attractive" and "Pictures on social media (YouTube, Instagram, Pinterest, Facebook) are an important source of information about hair and being attractive." Example items for the pressure subscale are "I've felt pressure from TV or magazines to change my appearance" and "I've felt pressure from TV and magazines to have a smoother hair texture." Example items from the internalization/hair subscale are "I compare my hair to the hair textures and styles of people who are on TV" and "I would like my hair to look like the models who appear in magazines." Example items from the internalization/appearance subscale are "I wish I looked like the models in music videos" and "I compare my appearance to the appearance of people in magazines." A higher score on each subscale indicates a greater tendency of one to endorse societal appearance ideas. 
Thompson et al. (2004) found the internal consistencies for the SATAQ-3 subscales to be excellent. The scales reliability, when tested in a large clinical samples of women, rendered Cronbach's Alpha ranging from .77 to .97 with alpha coefficients for each subscale, indicated in one study, as followed: Information $=.94$, Pressures $=.94$, Internalization-Athlete $=.89$, an Internalization-General $=.92$.

The original scale is intended, and has been used, to measures one's endorsement of societal ideas pertaining to appearance and body image. I, the researcher of this present study, modified this questionnaire, substituting questions that originally asked about body image to those that asked about hair and using the focus of original items to focus attention on hair. For example, "I would like my body to look like the models who appear in magazines" was revised to "I would like my hair to look like the models who appear in magazines" and "I've felt pressure from TV and magazines to be thin" was revised to “I’ve felt pressure from TV and magazines to have a smoother hair texture".

I find these modifications to still warrant the instrument valid as the questions revised continue to measure essentially what the instrument is intended to measure: sociocultural attitudes about aspects of appearance. These aspects of appearance be they appearance/body to appearance/hair is not a far leap in assumption as the scale assess internalization, media pressure, and media information sources related to appearance. Presently there are no instruments that measure sociocultural attitudes about hair texture; nor have any quantitative studies been conducted akin to this present study.

\section{Internalized Racial Oppression Scale}

The IROS for black individuals is composed of 28 items and intended to measure the degree to which racial oppression is internalized and replicated by Black individuals 
in the United States. An exploratory factor analysis suggested a five-factor solution: Belief in the Biased Representation of History (BRH), Devaluation of the African Worldview and Motifs (DAW), Alteration of Physical Appearance (APA), Internalization of Negative Stereotypes (INS), and Hair Change (HC) (Bailey, Chung, Williams, Singh, $\&$ Terrell, 2011). Participants are asked to read questions about their thoughts, beliefs, and behaviors and then respond to them using a 5-point Likert scale ranging from 1 (strongly disagree) to 5 (strongly agree). The instruments subscales-BRH, APA, INS, and $\mathrm{HC}$ - evaluate four dimensions of IRO. Example items from the BRH subscale are "African people have no written history" and "The first mathematicians and scientists were European." Example items from the APA subscale are "I wish I looked more white" and "Lighter skin is more attractive." Example items from the INS subscale are "Most criminals are Black men" and "Black women are confrontational." Example items from the HC subscale are "Straight hair is better than my natural hair texture" and "I prefer my hair to be natural." Higher scores on the instrument as a whole or on each subscale indicate higher levels of IRO.

Bailey et al. (2011) found that the IROS has high internal consistency. Cronbach's Alpha for the entire scale was .87 with alpha coefficients for each subscale as followed: $\mathrm{BRH}=.77, \mathrm{APA}=.72, \mathrm{INS}=.81$, and $\mathrm{HC}=.69$. Based on the Cronbach's Alpha $(\alpha=$ .87) the IROS was seen as reliable instrument for this present study.

The Multigroup Ethnic Identity Measure

The MEIM has become one of the most widely used measures of EI. This selfreported measure was designed for use with members of various ethnic groups and purported to capture aspects of EI equally applicable across ethnic groups. The challenge 
has been whether the MEIM is able to measure and identify the EI of multiple groups at a time and whether it contains a two factor or three factor subscale to best describe what it presumes to measure.

The MEIM is composed of 15 items and intended to measure multiple components of EI including: ethnic self-identification, degree of involvement and participation in social activities and cultural traditions with members of one's ethnic group, sense of belonging to an ethnic group, attitudes toward one's group, and EI achievement (Phinney, 1992). Of the 15 items 12 are on a Likert scale. The other three include open ended questions that elicit ethnic self-identification (self-label) as well as parental labels for mother and father. Participants are asked to read questions about their EI beliefs and behaviors and then respond to them using a 4-point Likert scale ranging from 1 (strongly disagree) 4 (strongly agree). The Phinney version used in this research contains only two subscales: EI search (EI Search) and EI affirmation, belonging, commitment (EI ABC). Example items of the EI Search subscale are "I have spent time trying to find out more about my ethnic group, such as its history, traditions, and customs" and "I am active in organizations or social groups that include mostly members of my own ethnic group." Example items of the EI ABC subscale are "I have a lot of pride in my ethnic group" and "I feel a strong attachment towards my own ethnic group." Higher scores on the total instrument or subscales indicate greater EI identification.

Phinney (1992) found that the MEIM, over the course of many studies, has consistently shown good reliability with alphas ranging in the .80 ranges when used with various ethnic groups and ages. Current revisions to the MEIM have rendered a twofactor subscale, which will be the focus in this study, that include (1) EI Search and (2) 
EI ABC. Ethnic identity search refers to behaviors that encompass seeking out ones ethnic background, heritage, and people. Affirmation, belonging, and commitment refer to how close and inclusively apart of individuals feel to their ethnic group.

\section{The General Self Efficacy Scale}

The GSE, created by Ralf Schwarzer and Matthias Jerusalem, is a onedimensional scale composed of 10 items that assess a general sense of perceived selfefficacy with the aim in mind to predict coping with daily life and adaptation after experiencing all kinds of stressful life events. Participants are asked to read statements about perceived self-efficacy and then respond to them using a 4-point Likert scale ranging from 1 (not at all true) to 4 (exactly true). This scale contains no subscales. Example items on the GSE are "It is easy to stick to my aims and accomplish my goals" and "I can solve most problems if I invest the necessary effort." Higher scores on the instrument indicate higher levels of perceived self-efficacy, coping, and adaptation to various life aspects.

The GSE is available in 33 different languages and is designed for the general adult population, including adolescents. The GSE has been tested in samples from 23 nations with Cronbach's Alphas ranging from .76 to .90 , with a majority being in the high .80 s. Criterion related validity is documented in numerous correlation studies where positive coefficients were found with favorable emotions, dispositional optimism, and work satisfaction. Negative coefficients were found with depression, anxiety, stress, burnout, and health complaints. The GSE has been used internationally with success for two decades. It is suitable for a broad range of applications including as an indicator of quality of life at any point in time (Schwarzer \& Jerusalem, 1995). 


\section{Data Collection}

To my knowledge, no harm came to any research participant in the process of this study. Surveys were anonymous and the subject matter being studied was not of such a sensitive nature to induce distress or psychological harm. Participants were informed of the nature of the surveys before they elected to take them and were free to discontinue at any point in time. The primary researcher's contact information and the contact information of the dissertation chair were provided to participants in the event that they had any questions or concerns.

The sample consisted of 322 participants. Participants were recruited via QuestionPro, Andrew's University student body, and social media, specifically Facebook. A total of 200 participants were recruited from QuestionPro and 122 from Facebook and a small literal arts university's student population in Southwest Michigan via email and sharing the research survey link.

Upon opening the survey, participants were introduced to the study and informed of any potential risks and benefits. They were also informed of their right to discontinue the survey at any time. By clicking on a particular button, at the end of the informative section, participants acknowledged that they had read and understood the introductory information, were consenting to take the survey, and that they met the requirements, including being female, age 18 and over, growing up in the United States, and identified as AA or Black. Following this, demographic information was gathered including age, education level, income, marital status, employment status, country of birth, where they were raised in the United States, ethnicity, parental ethnicity, and various questions 
pertaining to conformity, skin tone, and hair texture. Participants then responded to the SATAQ-3 Modified, IROS, MEIM, and GSE.

\section{Treatment of Data}

All survey answers were kept confidential. QuestionPro certifies accurate and secure transfer of data by transmitting it directly to the Statistical Package for Social Sciences, $24^{\text {th }}$ Edition (SPSS24). This is done in efforts to reduce human error.

QuestionPro's Respondent Anonymity Assurance (RAA) feature was used to safeguard anonymity. No identifying information linked participants to their survey responses. In addition, RAA assigns participants a code and removes all identifying information that QuestionPro has about its participants. Internet protocol address information was collected by QuestionPro to ensure that the same person did not take the survey twice, however this information is erased and excluded from the final data set and SPSS24 file given.

\section{Data Analysis}

Survey data collected was analyzed using SPSS24 and Analysis of a Moment Structures (AMOS) 21 computer software. I analyzed my data using SEM in order to determine the strength of the relationship between the aforementioned variables. Structural equation modeling was a good fit for my study as it allowed me to examine my hypothesized model based on theory with actual data, which became the basis of my empirical model. Structural equation modeling also allowed for the analysis of both observed and unobserved (latent) variables. Compared to other regression methods, SEM 
has a greater ability to account for measurement error of variables. It also allows for the parallel analysis and evaluation of both dependent and independent variables.

Before assessing model fit, I screened for any missing data, and removed such cases. There was only one such case found. It was deleted from the data set.

\section{Summary}

In this chapter, the research methodology for the present study was described. The study used several statistical analysis techniques via SPSS24 and AMOS to answer the two research questions that guided this study. These methods included descriptive statistics and SEM, an extension of multiple regression that employs visual structural modeling to represent causal types of relationships. The examined relationships were between the independent variables IRO, EI, and GSE with the dependent variable MI about hair texture. The research design was defined in this chapter and the population and sample were identified. Research questions and hypotheses were proposed and the research variables were defined. Specifically, the following variables were defined: MI, hair texture, IRO, EI, and self-efficacy. The instruments that were used to measure these variables were identified. The data collection procedures, and data analysis procedures were described.

Chapter 4 will present the results of my research and Chapter 5 will provide a summary and discuss recommendations in connection with the research results and the continuation of research moving forward. 


\section{CHAPTER 4}

\section{RESULTS}

\section{Introduction}

The purpose of this research was to examine the impact of MI about hair texture on IRO, EI, and GSE. In this chapter, the demographic characteristics of the participants are described. Results for each research question are then presented. Structural Equation Modeling was used to test this hypothesis and analyze the relationships between the variables. This chapter will present a description of this study's sample, a description of the study's variables, and the results of the SEM analysis.

\section{Description of the Sample}

The researcher's intention was to collect survey responses from 400 individuals, but due to an "error" in the survey company's system, survey results were obtained from only 200 participants via QuestionPro with the remaining 122 participants being recruited from a small university and online media sources. A total of 322 individuals completed the surveys with each participant remaining in the study. Demographic information about the sample is presented in Table 2 . The sample consisted of $100 \%$ females $(N=322)$ raised in the United States. More than half of the sample, 52\%, identified ethnically as AA $(N=168), 37.5 \%$ identified as Black $(N=121), 6.2 \%$ identified as Biracial $(N=20)$, and $4.3 \%$ identified as being Multiracial $(N=14)$. 
Table 2

Respondents' Demographic Characteristics

\begin{tabular}{|c|c|c|}
\hline Demographic & $N$ & $\%$ \\
\hline \multicolumn{3}{|l|}{ Age } \\
\hline \multicolumn{3}{|c|}{ Frequency by groups } \\
\hline $18-24$ & 81 & 25.2 \\
\hline $25-34$ & 108 & 33.5 \\
\hline $35-44$ & 62 & 19.3 \\
\hline $45-54$ & 32 & 9.9 \\
\hline $55-64$ & 24 & 7.5 \\
\hline $65-74$ & 12 & 3.7 \\
\hline $75+$ & 3 & 0.9 \\
\hline
\end{tabular}

Ethnicity

African American

$168 \quad 52$

Black

$121 \quad 37.5$

Biracial

$20 \quad 6.2$

Multiracial

$14 \quad 4.3$

Hair Texture (natural, unprocessed/unaltered)

$\begin{array}{lrr}1 & 14 & 4.3 \\ 2 & 12 & 3.7 \\ 3 \mathrm{a} & 17 & 5.3 \\ 3 \mathrm{~b} & 17 & 7.5 \\ 3 \mathrm{c} & 24 & 24.2 \\ 4 \mathrm{a} & 78 & 19.9 \\ 4 \mathrm{~b} & 64 & 23.3 \\ 4 \mathrm{c} & 75 & 11.8\end{array}$

Skin Tone

\begin{tabular}{rrr}
1 & 54 & 16.8 \\
2 & & \\
3 & 30 & 9.3 \\
4 & 31 & 9.6 \\
5 & 58 & 18.0 \\
6 & 71 & 22.0 \\
7 & 23 & 7.1 \\
8 & 32 & 9.9 \\
\hline
\end{tabular}


Of the $N=322$ respondents, 14 reported having hair type $1(4.3 \%), 12$ reported having hair type $2(3.7 \%), 17$ reported having hair type 3a (19.3\%), 24 reported having hair type $3 b(7.5 \%), 78$ reported having hair type $3 c$ (24.2\%), 64 reported having hair type $4 \mathrm{a}(19.9 \%), 75$ reported having hair type $4 \mathrm{~b}(23.3 \%)$ and 38 reported having hair type $4 \mathrm{c}(11.8 \%)$. In addition, responses indicated that $16.8 \%$ of participants identified as having skin tone resembling photo $1(N=54), 9.3 \%$ identified as having skin tone resembling photo $2(N=30), 9.6 \%$ identified as having skin tone resembling photo $3(N$ $=31), 18 \%$ identified as having skin tone resembling photo $4(N=58), 22 \%$ identified as having skin tone resembling photo $5(N=71), 7.1 \%$ identified as having skin tone resembling photo $6(N=23), 9.9 \%$ identified as having skin tone resembling photo $7(N$ $=32$ ), and $7.1 \%$ identified as having skin tone resembling photo $8(N=23)$ (Table 2 ; Appendix C).

\section{Instrument Reliability}

Table 3 lists the Cronbach's alpha for each of the instruments used in this study, associated with this study's collected data. All instruments demonstrated overall acceptable (over .7) levels of internal reliability; looking at the scales on a whole without evaluating subscales separately. This suggests that the scales each measured a single latent construct. The SATAQ-3 Modified demonstrated excellent internal reliability (over .9). The IROS and the MEIM demonstrated good internal reliability (over .8). The GSE demonstrated the lowest, but still acceptable, internal reliability at .774. 
Table 3

Internal Reliability

\begin{tabular}{lcc}
\hline Instrument & No. of Items & $\alpha$ \\
\hline SATAQ-3 Modified & $\mathbf{3 0}$ & $\mathbf{. 9 4 1}$ \\
Info & 10 & .680 \\
Pressure & 8 & .845 \\
Internal - Hair & 7 & .548 \\
Internal - Appearance & 5 & .625 \\
& & \\
IROS & $\mathbf{2 8}$ & $\mathbf{. 8 8 4}$ \\
INS & 7 & .870 \\
BRH & 8 & .542 \\
APA & 8 & .855 \\
HC & 5 & .207 \\
MEIM & $\mathbf{1 2}$ & $\mathbf{8 8 8}$ \\
EI Search & 5 & .802 \\
EI ABC & 7 & .893 \\
GSE & & $\mathbf{. 7 7 4}$ \\
\hline
\end{tabular}

\section{Variables Description}

Normality

All variables were screened for skewness and kurtosis to determine if the data set was well modeled by a normal distribution. Skewness describes the symmetry of the data distribution. Positive or negative values of skewness indicate bulking of the data on one end of the distribution continuum. Kurtosis describes clustering of scores toward the center of the distribution. In descriptive statistics both a symmetric distribution for skewness and a normal distribution for kurtosis have a value of 0 (Meyers, Gamst, \& Guarino, 2013). Table 4 represents the tests of normality. The variables measured for MI 
Table 4

Tests of Normality

\begin{tabular}{lrrrrrrr}
\hline & & \multicolumn{9}{c}{$95 \%$ Confidence } \\
& Skewness & Kurtosis & \multicolumn{2}{c}{ Interval } & \multicolumn{3}{c}{ Shapiro-Wilk } \\
& & & Lower & Upper & & & \\
& & & Bound & Bound & Statistic & $d f$ & Sig. \\
\hline SATAQ3 & -0.065 & -0.866 & 2.604 & 2.800 & 0.979 & 322 & .000 \\
IROS & 1.099 & 1.187 & 1.906 & 2.034 & 0.923 & 322 & .000 \\
MEIM & -0.598 & 0.703 & 3.065 & 3.182 & 0.967 & 322 & .000 \\
GSE & -0.905 & 1.599 & 32.284 & 33.431 & 0.937 & 322 & .000 \\
\hline
\end{tabular}

about hair (SATAQ-3 Modified), IRO (IROS), EI (MEIM), and GSE (GSE) were within acceptable limits (-1 to 1) for skewness and kurtosis (Meyers, Gamst, \& Guarino, p. 64).

Variable Means and Standard Deviations

The description of the variables, including mean, standard deviation, skewness, and standard error are reported in Table 5. For the variable MI about hair, scores were obtained from the SATAQ-3 Modified for information, pressure, internalization-hair, and internalization-appearance. For the variable IRO, scores were obtained from the IROS for INS, BRH, APA, and HC. For the variable EI, scores were obtained from the MEIM for EI Search and EI ABC. For the variable GSE, scores were obtained from the GSE.

Scores on the SATAQ3 range from 30-150 with a score of 30 indicating the lowest level of MI about hair texture and a score of 150 indicating the highest level of MI. Looking at the full scale, respondents averaged a score of 85 . Scores on the IROS range from 28-129 with a score of 28 indicating the lowest level of IRO and 129 indicating the highest levels of IRO. Looking at the full scale, respondents averaged a score of 62 . In this case the level of internalized racial oppression is at nearly $50 \%$. This 
Table 5

Variable Means and Standard Deviations

$\begin{array}{llllllll}\text { Variable } & \text { N } & \text { Min } & \text { Max } & \text { M } & \text { SD } & \text { Skewness } & \text { SE }\end{array}$

\section{Media Influence}

Information

Pressure

Internalization - Hair

Internalization - Appearance

SATAQ3 Modified Full Scale

Internalized Racial Oppression

INS

BRH

APA

$\mathrm{HC}$

IROS Full Scale

Ethnic Identity

EI Search

EI ABC

MEIM Full Scale

General Self-Efficacy

GSE Full Scale

$\begin{array}{rrrrrrr}322 & 10.00 & 50.00 & 28.90 & 7.13 & -0.17 & 0.14 \\ 322 & 8.00 & 40.00 & 22.44 & 8.04 & 0.02 & 0.14 \\ 322 & 7.00 & 35.00 & 20.33 & 5.03 & -0.05 & 0.14 \\ 322 & 5.00 & 25.00 & 13.46 & 4.36 & 0.28 & 0.14 \\ 322 & 30.00 & 150.00 & 85.14 & 21.38 & -0.06 & 0.14\end{array}$

$\begin{array}{rrrrrrr}322 & 7.00 & 32.00 & 11.56 & 5.36 & 1.51 & 0.14 \\ 322 & 8.00 & 37.00 & 20.49 & 4.27 & 0.34 & 0.14 \\ 322 & 8.00 & 36.00 & 14.65 & 6.13 & 1.31 & 0.14 \\ 322 & 5.00 & 25.00 & 15.39 & 3.05 & -0.59 & 0.14 \\ 322 & 28.00 & 129.00 & 62.09 & 15.19 & 1.57 & 0.14\end{array}$

322

322

322

322

5.00
7.00
12.00

20.00

$14.17 \quad 3.44$

$-0.45$

0.14

$\begin{array}{lll}28.00 & 23.32 & 3.92\end{array}$

$-0.78$

0.14

$48.00 \quad 37.48 \quad 6.42$

$-0.60$

0.14

$\begin{array}{lll}40.00 & 32.86 & 5.23\end{array}$

$-0.91$

0.14 
is high. To better understand this, let's consider suicide and depression. If an individual reported that they were simply "thinking" about suicide, even at a $1 \%$ level, the situation would be serious enough to require immediate intervention. If an individual reported that they were, say $20 \%$ depressed, they may require medication, or at minimum some form of intervention. The point is, internalized racial oppression, is harmful and therefore significant and impactful at any level or degree. If ethnic minorities, in this case AA and Black women, are adopting racist messages at the level of 50\%, that's a significant amount of influence by the media (the mean being $56 \%$ in this study) that resulted in internalized racial oppression levels of $48 \%$ for the average participant in this study.

Scores on the MEIM range from 12-48 with a score of 12 indicating the lowest level of EI and 48 indicating the highest levels of EI. Looking at the full scale, respondents averaged a score of 37 . Scores on the GSE range from 10-50 with a score of 10 indicating the lowest level of GSE and a score of 50 indicating the highest level of GSE. Respondents averaged a score of 33.

\section{Zero-Order Correlations}

Table 6 presents zero order (Pearson) correlations. As anticipated, MI about hair texture, was significantly correlated to IRO, EI, and GSE though more moderately than expected from this study's sample. The correlations being in moderate range suggests that each variable measured a distinct construct.

The significant correlations that existed between variables are theoretically consistent. Meaning, variables that were significantly corrected with MI about hair 
Table 6

Zero-Order Correlations

\begin{tabular}{|c|c|c|c|c|c|c|c|c|c|c|c|c|c|c|}
\hline & Info & Press. & $\begin{array}{l}\text { Internal } \\
\text { Hair }\end{array}$ & $\begin{array}{l}\text { Internal } \\
\text { App. }\end{array}$ & SATAQ3 & INS & BRH & APA & $\mathrm{HC}$ & IROS & $\begin{array}{c}\text { EI } \\
\text { Search }\end{array}$ & $\begin{array}{c}\mathrm{EI} \\
\mathrm{ABC}\end{array}$ & MEIM & GSE \\
\hline Pressure & & 1 & $.692^{* *}$ & $.698^{* *}$ & $.882 * *$ & $.168^{* *}$ & -.026 & $.262^{* *}$ & .088 & $.166^{* *}$ & $.313^{* *}$ & -.050 & $.137 *$ & $-.123^{*}$ \\
\hline $\begin{array}{l}\text { Internalization } \\
\text { Hair }\end{array}$ & & & 1 & $.807^{* *}$ & $.875^{* *}$ & $.288^{* *}$ & $.127^{*}$ & $.399^{* *}$ & $.238^{* *}$ & $.338 * *$ & $.265^{* *}$ & $-.117^{*}$ & .070 & $-.157^{* *}$ \\
\hline $\begin{array}{l}\text { Internalization } \\
\text { Appearance }\end{array}$ & & & & 1 & $.879 * *$ & $.315^{* *}$ & $.172^{* *}$ & $.450^{* *}$ & $.254^{* *}$ & $.383^{* *}$ & $.254^{* *}$ & $-.155^{* *}$ & .041 & $-.188^{* *}$ \\
\hline INS & & & & & & 1 & $.563^{* * *}$ & $.742^{* *}$ & $.378^{* * *}$ & $.867 * *$ & .017 & $-.247^{* *}$ & $-.142 *$ & $-.272^{* * *}$ \\
\hline $\mathrm{BRH}$ & & & & & & & 1 & $.508^{* *}$ & $.413^{* *}$ & $.776^{* *}$ & $-.234^{* * *}$ & $-.360^{* * *}$ & $-.346 * *$ & $-.264^{* *}$ \\
\hline APA & & & & & & & & 1 & $.457^{* *}$ & $.880 * *$ & .056 & $-.220^{* *}$ & -.104 & $-.186^{* *}$ \\
\hline $\mathrm{HC}$ & & & & & & & & & 1 & $.647 * *$ & $-.115^{*}$ & $-.250^{* *}$ & $-.214 * *$ & $-.114^{*}$ \\
\hline IROS & & & & & & & & & & 1 & -.071 & $-.330^{* *}$ & $-.240 * *$ & $-.266 * *$ \\
\hline GSE & & & & & & & & & & & & & & 1 \\
\hline
\end{tabular}

**. Correlation is significant at the 0.01 level (2-tailed). *. Correlation is significant at the 0.05 level (2-tailed). 
texture were positively correlated (e.g., higher MI was correlated with higher IRO). There were also significant negative correlations among variables (e.g., higher IRO was correlated with lower GSE).

\section{Hypothesis Testing}

The first hypothesis of this study was that the theoretical covariance matrices would be equal to the empirical covariance matrices. The second hypothesis of this study was that the reproduced, or empirical covariance matrices would differ between groups of women based on ethnic identification, hair texture, and skin tone. For the first hypothesis the null hypothesis was rejected. As such, the models were respecified based on theory and modification indices. These modifications are detailed below. The fit indices for the original and fitted models are presented in Table 7.

For the second hypothesis, the respecified model was used to test for differences between groups of women based on ethnic identification, hair texture, and skin tone. The between groups, based on ethnic identification, hair texture, and skin tone, did differ in several ways. Those results are discussed below and detailed in Table 8 .

\section{Research Question One}

Is the hypothesized covariance matrix equal to the observed covariance matrix collected from the data?

\section{Original Model}

The conceptual model previously detailed in Chapters 1 and 3 include the predictor variable MI about hair texture, and the outcome variables IRO, EI, and GSE. A 
Table 7

Chi-Square and Goodness of Fit Indices for Hypothesized and Respecified Models

\begin{tabular}{|c|c|c|c|c|c|c|c|c|}
\hline Model & $\mathbf{X}^{2}$ & $P$ & $d f$ & GFI & NFI & CFI & RMSEA & SRMR \\
\hline Original & 170.35 & .000 & 42 & 0.83 & 0.78 & 0.82 & .144 & .14 \\
\hline Respecified & 76.73 & .000 & 36 & 0.96 & 0.96 & 0.98 & .059 & .05 \\
\hline \multicolumn{9}{|c|}{ Between Groups, Respecified } \\
\hline \multicolumn{9}{|c|}{ Ethnic Identification } \\
\hline African American & 71.07 & .000 & 36 & 0.93 & 0.93 & 0.97 & .076 & .06 \\
\hline Black & 44.71 & .151 & 36 & 0.94 & 0.93 & 0.96 & .045 & .05 \\
\hline \multicolumn{9}{|l|}{ Hair Texture } \\
\hline 1 to 4 & 44.91 & .146 & 36 & 0.89 & 0.91 & 0.98 & .061 & .06 \\
\hline 5 to 8 & 76.35 & .000 & 36 & 0.95 & 0.94 & 0.97 & .066 & .05 \\
\hline \multicolumn{9}{|l|}{ Skin Tone } \\
\hline 1 to 4 & 54.65 & .024 & 36 & 0.95 & 0.95 & 0.98 & .055 & .05 \\
\hline 5 to 8 & 66.72 & .001 & 36 & 0.93 & 0.91 & 0.96 & .076 & .06 \\
\hline
\end{tabular}

Note. X2=Chi square test; $d f=$ degrees of freedom; GFI=Goodness of Fit Index; NFI= Normed Fit index, $\mathrm{CFI}=$ Comparative Fit Index; RMSEA= Root Mean Square Error of Approximation; SRMR=Standardized Root Mean Square Residual 
Table 8

Standardized Regression Weights of Variables Between Groups

\begin{tabular}{|c|c|c|c|c|c|c|c|c|c|}
\hline \multicolumn{2}{|c|}{ Variable (s) } & $\beta$ & $r^{2}$ & $\beta$ & $r^{2}$ & $\beta$ & $r^{2}$ & $\beta$ & $r^{2}$ \\
\hline \multirow[t]{10}{*}{ MI } & & IRO & $\%$ & EI & $\%$ & EI ABC & $\%$ & GSE & $\%$ \\
\hline & Ethnic & & & & & & & & \\
\hline & AA & .526 & 28 & .441 & 19 & -.610 & 37 & -.522 & 27 \\
\hline & Black & .421 & 18 & .663 & 44 & -.532 & 28 & -.431 & 19 \\
\hline & Hair T & & & & & & & & \\
\hline & 1 to 4 & .623 & 39 & .628 & 39 & -.638 & 41 & -.401 & 16 \\
\hline & 5 to 8 & .432 & 19 & .482 & 23 & -.542 & 29 & -.465 & 22 \\
\hline & Skin T & & & & & & & & \\
\hline & 1 to 4 & .441 & 19 & .563 & 32 & -.563 & 32 & -.432 & 19 \\
\hline & 5 to 8 & .524 & 27 & .407 & 17 & -.494 & 24 & -.442 & 20 \\
\hline
\end{tabular}

IRO

Ethnic ID

AA

$-.043 \quad .002$

Black

$-.526 \quad 28$

Hair Texture

1 to 4

$-.182 \quad .03$

5 to 8

$-.239 \quad .06$

Skin Tone

$\begin{array}{lll}1 \text { to } 4 & -.271 & .07 \\ 5 \text { to } 8 & -.215 & .05\end{array}$

EI

Ethnic ID

AA

$.750 \quad 56$

Black

$.335 \quad 11$

Hair Texture

$.558 \quad 31$

1 to 4

$.541 \quad 29$

Skin Tone

1 to 4

$.508 \quad 26$

5 to 8

$.591 \quad 35$ 
direct predictor path was drawn from MI about hair texture to IRO, as research shows that greater exposure to majority MIs can negatively impact Black autonomy, and sense of self; especially when minorities adopt the views of the majority about themselves (Allen, 2001; Fujioka, 2005; Ward, 2004). A direct path was also drawn from MI about hair texture to EI, as research shows that dominant culture MIs that devalue physical features common to African people can be damaging (Azibo, 1989; Onwuachi-Willig, 2016; Vargas \& Kingsbury, 2016). Finally, from the conceptual model, a direct path was drawn from MI about hair texture to GSE, as research shows that propagated influences

of the media's prejudice in representation and cultural practices fuels the blatant rejection of African hair types and textures causing disruption of progress in both the classroom and the work place for AA and Black females (Andrews, 2014; Byrd \& Tharps, 2001; Dossou, 2013; Rhodan, 2014).

The hypothesized model was a poor fit for the data. The Chi-square for the original model was $170.35(d f=40)$ with a probability level of $<.000$. Therefore, the model was evaluated on the basis of fit indices for revision. The criteria used to determine acceptable model fit was: GFI ( $\geq$. 90), NFI $(\geq .95)$, CFI $(\geq .95)$, RMSEA $(\leq$ $.08)$; Cut-off values were determined according to the recommendations discussed in $\mathrm{Hu}$ \& Bentler (1999) as well as Meyers, Gamst, and Guarino (2013). The fit indices for the hypothesized model were as follows: $\mathrm{GFI}=.83, \mathrm{NFI}=.78, \mathrm{CFI}=.82, \mathrm{RMSEA}=.14$.

\section{Fitted Model}

Modification indices were examined in order to determine how the model could be revised to be more accurate and still be theoretically consistent. The final model is depicted in Figure 2. Pearson $r$ or correlation coefficient is denoted here within as $r$. 


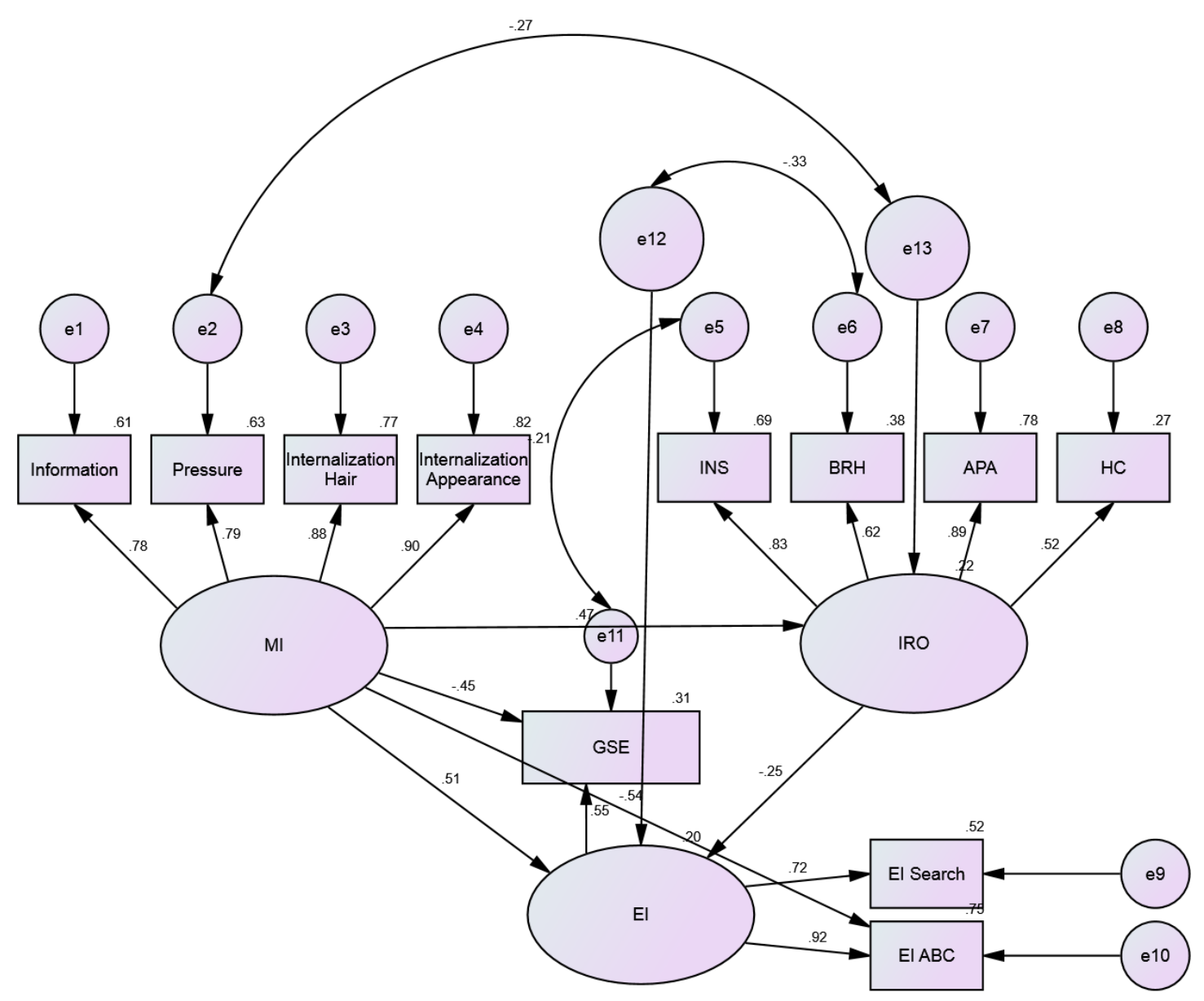

Figure 2. Respecified Model.

Effect sizes were determined according to the recommendations of Rosenthal (1996).

Therefore, the following thresholds were used: small $=.10$; medium $=.30$; large $=.50$; very large $=.70$. First, no items were removed.

Second, suggested correlations that were theoretically consistent were added. A path was added from MI to EI ABC, based on the theory that MI directly affects one's EI in terms of affirmation, belonging, and commitment. A direct path was added from IRO to EI, which was negative, based on the theory that increased IRO effects various aspects 
of an individual's EI, by decreasing positive EI achievement. A direct path was added from EI to GSE, based on the theory that EI impacts an individual's GSE based on how one perceives themselves and their ethnic group. Also, three unexplained correlations were added from the following error terms: GSE $\leftarrow \rightarrow \mathrm{INS}$, GSE $\leftarrow \rightarrow \mathrm{BRH}$, and IRO $\leftarrow \rightarrow$ Pressure, meaning these variables are associated in some way though the negative or positive directional pathways are unknown.

The resulting model was well fit to the data. The chi-square for the fitted model was significant at $76.73, d f=36, \mathrm{p}=.000$; and therefore the null hypothesis is typically rejected; however, chi-square is known to be sensitive to sample size and so in this case the GFI, NFI, CFI, and RMSEA better helped determine the model fit. The GFI and NFI both indicated a strong fit at .96 and the CFI also indicated a strong fit at .98 . The RMSEA indicated an improved, and good fit at .059 , using the $\leq .08$ cut-off value.

\section{Explanation of the Fitted Model}

Pertaining to the respecified model the following direct pathways will be explained: MI to IRO, MI to EI, MI to EI ABC, MI to GSE, IRO to EI, and EI to GSE. First, as expected, the fitted model indicated that MI about hair texture was positively correlated with IRO $(r=.47)$, the shared variance between the two variables was about $22 \%$. This suggests that as MI about hair texture increases, IRO also increases. When looking at the between groups in Table 8, the impact of MI on IRO was two times greater in AA women $(r=.53)$ than in Black women $(r=.42)$ based upon the standard error (Table 8).

Second, the fitted model indicated that MI about hair texture was positively correlated with EI $(r=.51)$, the shared variance between the two variables was about 
$26 \%$. This suggests that as MI about hair texture increases, EI also increases. This result is nonintuitive, however previous research has shown that EI might still be increased from other images within the media such as prominent AA and Black athletes and entertainers that would increase one's desire to ethnically identify with their group and solidify membership to that group (Chambers et al., 1994; Wade, 1996). Hence, the fitted model also indicated that MI about hair texture was negatively correlated with $\mathrm{EI}$ ABC ( $r$ $=-.54)$, the shared variance between the two variables was about $29 \%$.

Third, the fitted model indicated that MI about hair texture was negatively correlated with GSE ( $r=-.45)$, the shared variance between the two variables was about 20\%. This suggests that as MI about hair texture increases, self-efficacy decreases. This is consistent with the research. AA and Black women are constantly observing images that glorify hair textures that do not look like theirs. They are told through these images that certain hair textures and lengths can afford one the privileges of academic, job, and relational success and upward mobility. Those who do not fit the mold of the propagated Eurocentric beauty standards of majority culture are forced to face the real or perceived reality that they cannot achieve and obtain a certain levels of success without strict conformity to beauty paradigms that are essentially unobtainable, supported by no laws of nature, and ultimately exclude them (Allen, 2016; Andrews, 2014; Hunter, 2013; White, 2008). When looking at the between groups, no group was effected more significantly than another, however there were greater effect correlations as it pertains to AA women $(r=-.50)$, hair textures 5 to $8(r=-.50)$, and skin tone $(r=-.44)$. The effect sizes of AA women and hair textures 5 to 8 were large. 
Fourth, the fitted model indicated that IRO was negatively correlated with EI ( $r=$ -.25 ), the shared variance between the two variables was about $6 \%$. The path coefficient between IRO and EI in this sample suggested a small effect size, but is consistent with the aforementioned research and suggests that as IRO increases, EI decreases. Internalized Racial Oppression is the adaptation and embracing of racist messages by ethnic minorities that result in self-hatred. An increase in IRO would cause a decrease in EI because AA and Black women would be less inclined to ethnically identify with their own ethnic group, which lessens one's EI ABC to their ethnic group. This in turn would result in less overall involvement and engagement with one's group that would drastically decrease EI achievement.

Fifth, the fitted model indicated that EI was positively correlated with GSE ( $r=$ $.55)$, the shared variance between the two variables was about $30 \%$. This suggests that as EI increases, GSE also increases. African Americans and Black women who are rightly, fairly, and accurately portrayed within media see themselves positively represented and avoid subscribing to the constant transmission of negative images or the one-sided Eurocentric image of what is, and who is worthy, good, and beautiful. This allows ethnic minorities to embrace their ethnicity though search and knowledge, affirmation and commitment, and enjoy a sense of belonging to their group which essentially boosts one's self-esteem and in turn their GSE, in that there is a belief that all things are possible. Ethnic minorities begin to see themselves as worthy, capable, and value not only their heritage but themselves as a God given right via their humanity and not solely through the dark biased lens of propagated Eurocentric beauty standards. When looking at the between groups, Table 8 shows that this path correlation is four times more strongly 
indicated in AA women $(r=.80)$ than Black women $(r=.34)$. For the AA model, this path was statistically significant and the effect size was very large. The EI to GSE correlation was also more strongly correlated, four times as much, in women with skin tones 5 to $8(r=.59)$ than skin tones 1 to $4(r=.51)$.

Sixth, and lastly, Figure 2 shows the causal effects of the respecified model. In the respecified model, the combination of MI about hair texture and EI account for $50 \%$ of the variance in GSE. EI was the strongest predictor of $\operatorname{GSE}(\beta=.55)$.

\section{Research Question Two}

Does ethnic identification, hair texture, or skin tone influence the fit of the empirical model?

\section{Ethnic Identification}

When examining the respecified model to determine if results varied by ethnic identification in Figure 3 and Figure 4 (Table 7), only two groups were looked at, AAs ( $N$ $=168)$ and Blacks $(N=120)$. The chi-square for the fitted model of the AA identified ethnic group was significant: $71.01, d f=36, p=.000$. Therefore we look to the GFI, NFI, CFI, and RMSEA. The GFI and NFI indicated a good fit at .93 and the CFI indicated a strong fit at .97 . The RMSEA was just below the $\leq .08$ cut-off at .076 , and therefore a good fit. The chi-square for the fitted model of the Black identified ethnic group was $44.71, d f=36, \mathrm{p}=.151$ indicating a non-significant chi-square and therefore the model is a strong fit. These results indicate that ethnic identification did not influence the fit of the empirical (respecified) model. 


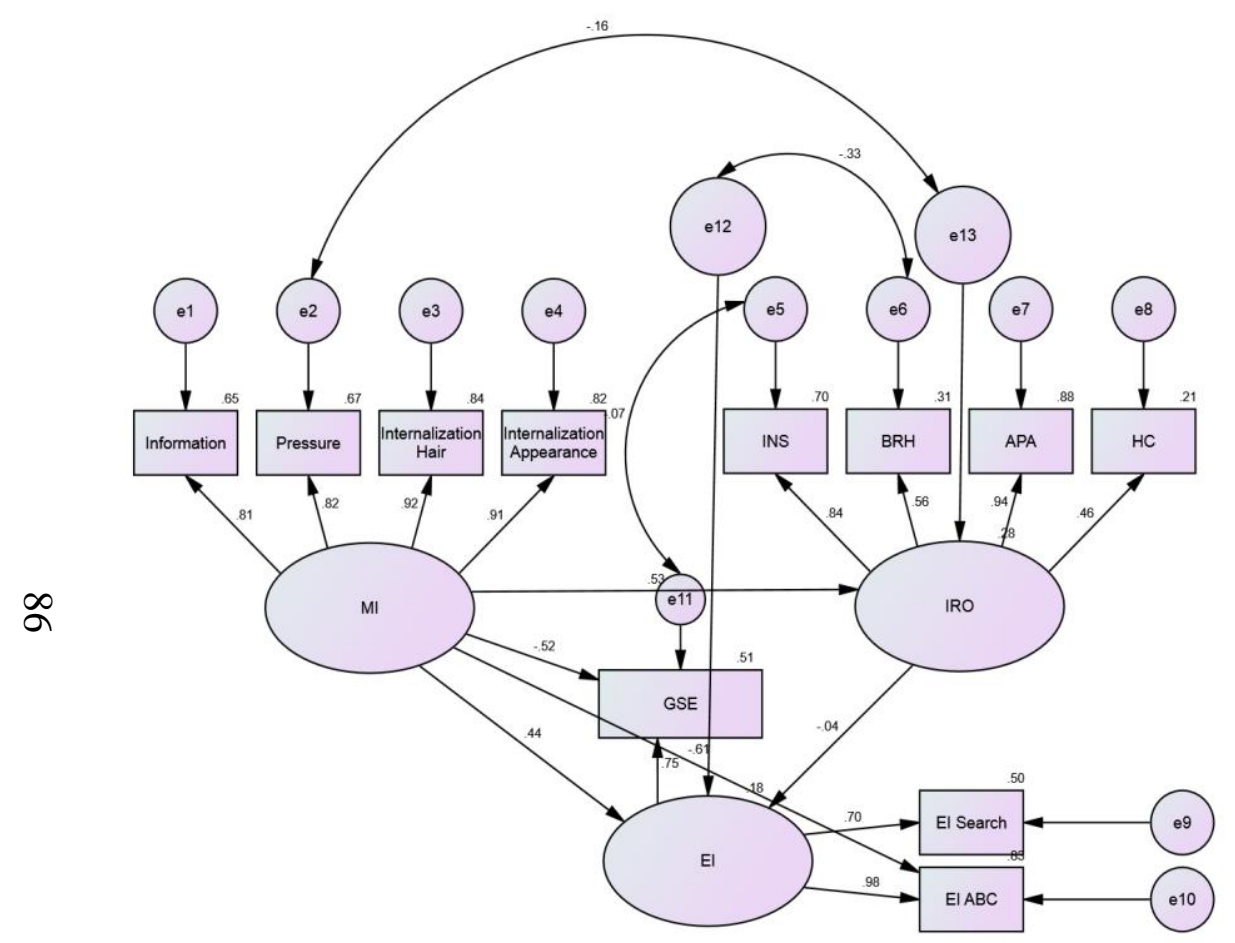

Figure 3. Respecified Model: African American.

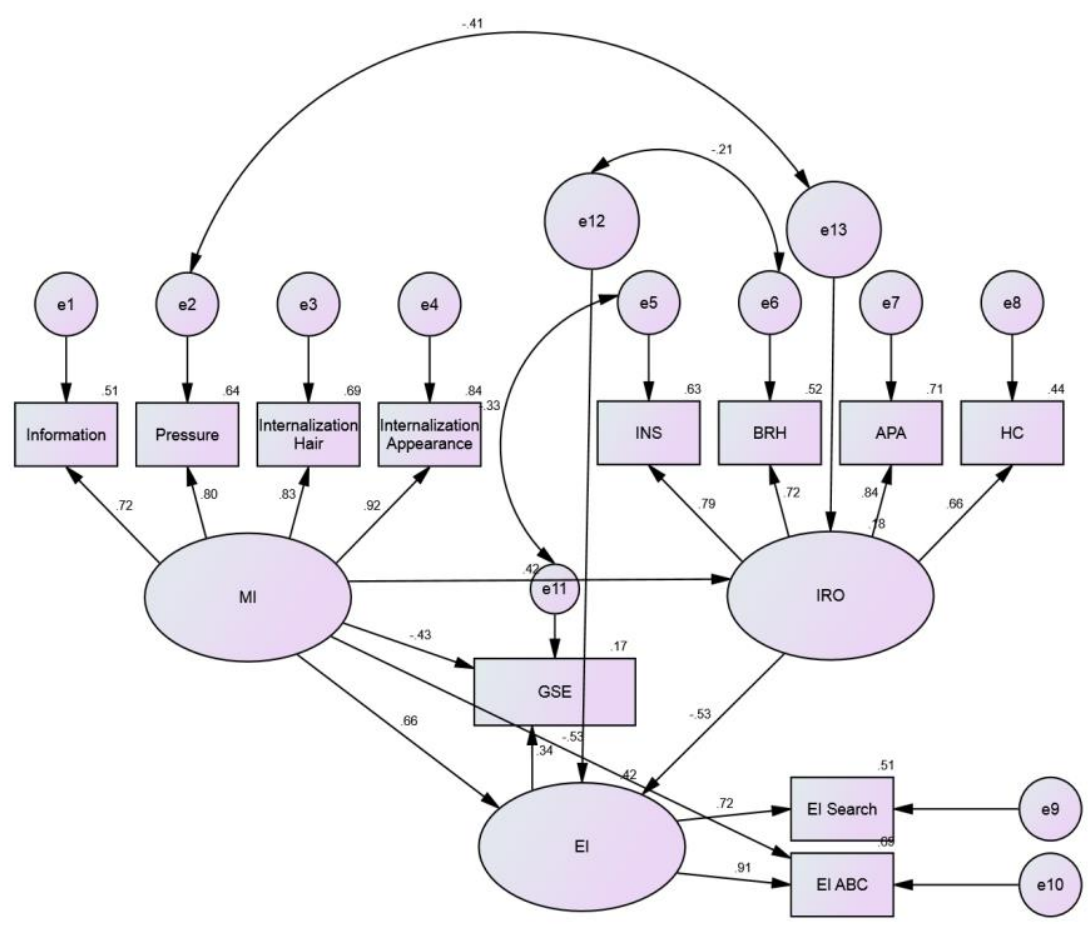

Figure 4. Respecified Model: Black. 
African American and Black are used interchangeably. However Black is the overarching term, umbrella, which is inclusive of AAs. Black often pertains to individuals who identify with having African heritage however may not identify as being American because they claim another country of origin as their home; however this does not mean that they were not raised fully or even partially in America therefore being influenced by American society. In this respect, Black is not a color or a skin tone but a particular ethnicity, which includes the African-American and Black experience because of the particular ancestry and associated combined physical, facial, skin, and hair features. In this study, as where one was socialized is an important factor of this study, those who identified as Black were raised in the United States thereby being socially influenced in the content of American and majority culture.

In comparing the two groups, the data in Table 8 indicated several significant differences between the groups as it pertains to AA and Black ethnically identified women. First, MI and IRO was more strongly correlated among AA women $(r=.53)$ than black women $(r=.42)$ suggesting a large effect size for AA women with a shared variance of $28 \%$ between the two measured variables. Second, MI and EI was more strongly correlated among Black women $(r=.66)$ than AA women $(r=.44)$ suggesting a large effect size for Black women with a shared variance of $44 \%$ between the two variables. Lastly, EI and GSE was more strongly correlated among AA women $(r=.75)$ than black women $(r=.34)$ suggesting a very large effect size for AA women with a shared variance of $56 \%$ between the two variables.

In addition, when looking at the direct path from MI to EI ABC, in Figure 2, the correlation is negative $(r=-.54)$. Table 8 shows that this path correlation was more 
strongly indicated in AA women $(r=.61)$ with a shared variance between the variables of $37 \%$; hair textures 1 to $4(r=.63)$ with a shared variance between the variables of $41 \%$; and skin tones 1 to $4(r=.56)$ with a shared variance between the variables of $32 \%$. The effect size of all three between groups is large. This suggests, as in the research, where one falls on the hair texture and skin tone continuum plays a significant role in personal and other perceptions of self (Badillo, 2001; Greene et al., 2000; Harrison \& Thomas, 2009; Patton, 2006; White, 2008). Black women are more able to identify ethnically, in fact three times more than AA women, in spite of MI about hair textures because certain Black women may not identify as African or even as American though being raised in America, and feel immune or removed from particular aspects of American culture. Also, this particular group that possesses hair textures (1 to 4) and skin tones (1 to 4), which are

more similar to the majority are advantaged and fare better than those who do not possess these hair textures and skin tones, which affords one the ability to see themselves within the paradigms of majority culture and maintain aspects of their EI (Bond \& Cash, 1992; Gatewood, 1988; Wade, 1996).

\section{Hair Texture}

There were eight different hair typings, which were divided into two groups. The first group $(N=67)$ ranged from 1 to 4 (hair typing $1,2,3 \mathrm{a}, 3 \mathrm{~b})$ which were straight or loosely coiled and the second group $(N=255) 5$ to 8 (hair typing $3 \mathrm{c}, 4 \mathrm{a}, 4 \mathrm{~b}, 4 \mathrm{c}$ ) which were more tightly coiled (Appendix C). Typically, when using AMOS to look at groups, there should be a minimum of at least 100 samples. In the case of the first hair typing group ranging from 1 to 4 , this data set only had $N=67$. 
The chi-square for the fitted model of the first hair texture group 1 to 4 (Table 7) was 44.91, $d f=36, p=.146$ indicating a non-significant chi-square and therefore the model was a good fit. The chi-square for the fitted model of the second hair texture group 5 to 8 was $76.35, d f=36, p=.000$. The GFI indicated a good fit at .95 , the NFI indicated a good fit at .94, and the CFI indicated a strong fit at .97. The RMSEA was .066 and therefore a good fit. These results indicate that hair texture did not influence the fit of the empirical (respecified) model.

In comparing the two groups in Figure 5 and Figure 6, the data in Table 8 indicated two significant differences between the groups as it pertains to hair textures. First, MI and IRO was more strongly correlated among hair textures 1 to $4(r=.62)$ with a shared variance between the variables of 39\%; than textures 5 to $8(r=.43)$ with a shared variance between the variables of $19 \%$. The effect size among hair textures 1 to 4 was large. Second, MI and EI was more strongly correlated among hair textures 1 to 4 ( $r$ $=.63)$ with a shared variance between the variables of $39 \%$; than textures 5 to $8(r=.48)$ with a shared variance between the variables of $28 \%$.

\section{Skin Tone}

There were eight different skin tones from which participants selected from as resembling their own, and these were also divided into two different groups. The first group $(N=173)$ ranged from 1 to 4 (skin tones $1,2,3,4)$ which were lighter in hue and the second group $(N=149) 5$ to 8 (skin tones $5,6,7,8)$ which were darker in hue (Appendix C). The chi-square for the fitted model of the first skin tone group (1 to 4 ) was 54.65, $d f=36, p=.024$ indicating a non-significant chi-square and therefore the model was a good fit. The chi-square for the fitted model of the second skin tone group (5 to 8 ) 


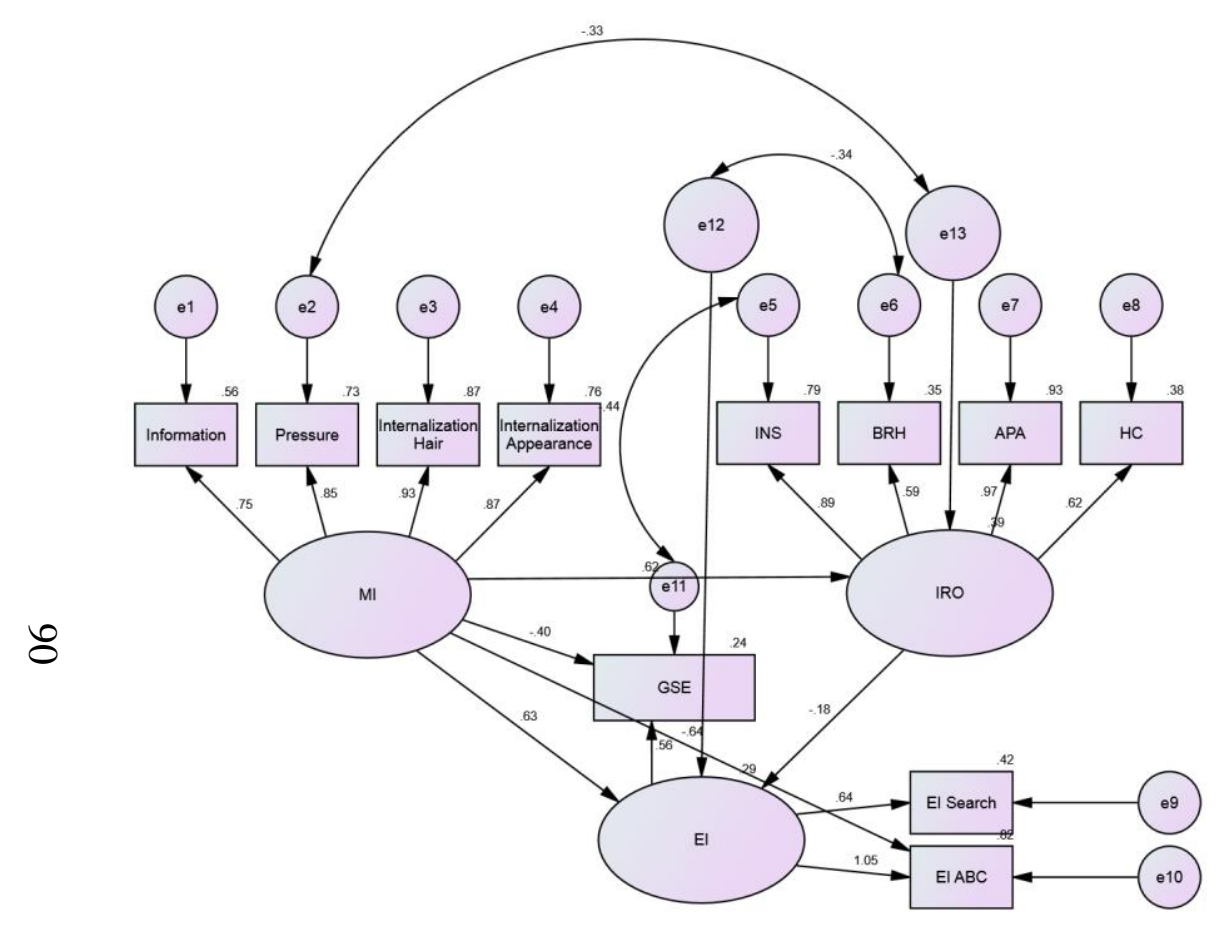

Figure 5. Respecified Model: Hair Textures 1 to 4.

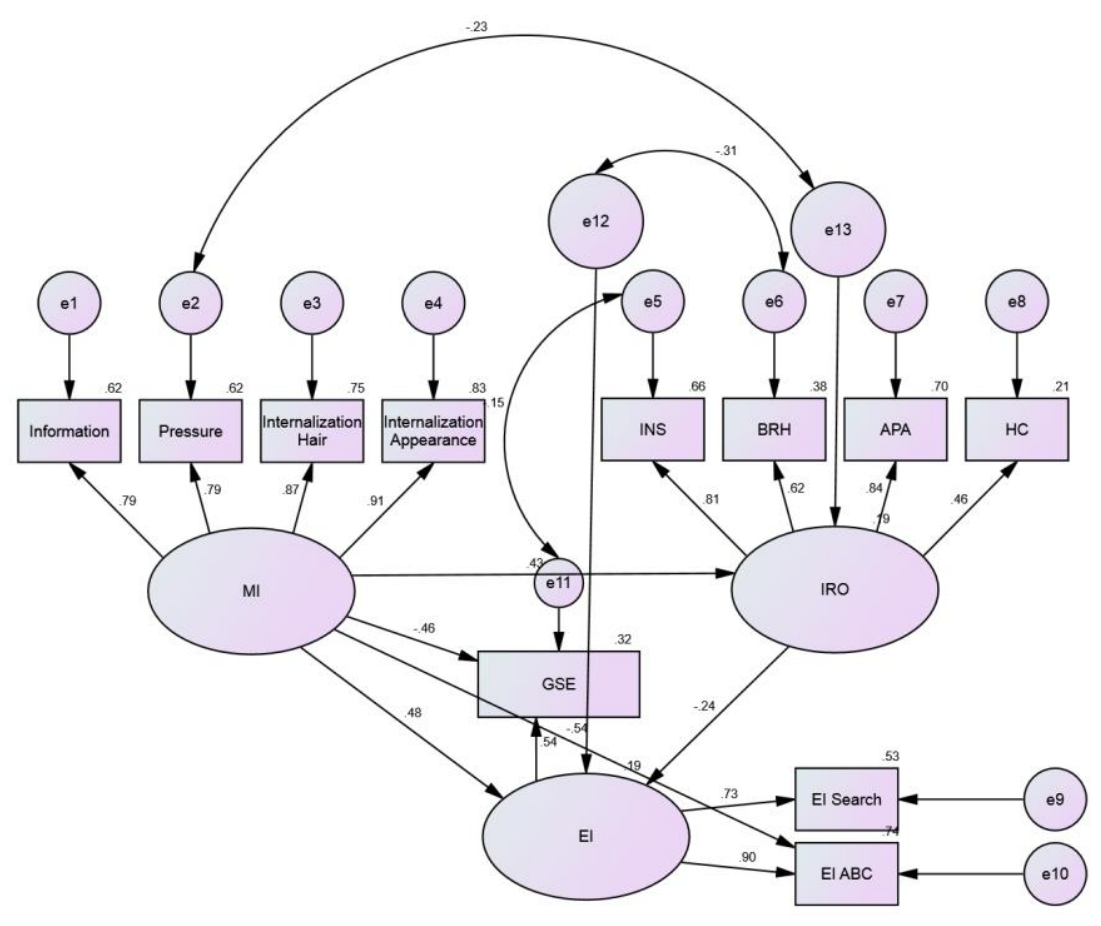

Figure 6. Respecified Model: Hair Textures 5 to 8. 
was $66.72, d f=36, p=.001$. The GFI was acceptable at .93 , the NFI was acceptable at .91 , and the CFI indicated a good fit at .96. The RMSEA was just below the $\leq .08$ cut-off at .076 , and therefore a good fit. These results indicate that skin tone did not influence the fit of the empirical (respecified) model.

In comparing the two groups in Figure 7 and Figure 8, the data in Table 8 indicated two significant differences between the groups as it pertains to skin tones. First, MI and EI was more strongly correlated among skin tones 1 to $4(r=.56)$ with a shared variance between the variables of $32 \%$; than skin tones 5 to $8(r=.41)$ with a shared variance between the variables of $17 \%$. Second, EI and GSE was more strongly correlated among skin tones 5 to $8(r=.59)$ with a shared variance between the variables of $35 \%$; than skin tones 1 to $4(r=.51)$ with a shared variance between the variables of $26 \%$.

\section{Summary}

The results of this study were described in this chapter. First, characteristics of the sample including instrument reliability were described. Then, characteristics of the variables were described. Lastly, results of SEM analysis were shown and described, including steps taken to respecify the model. Chapter 5 will discuss the implications of the results in light of the research questions, recommendations, and future research. 


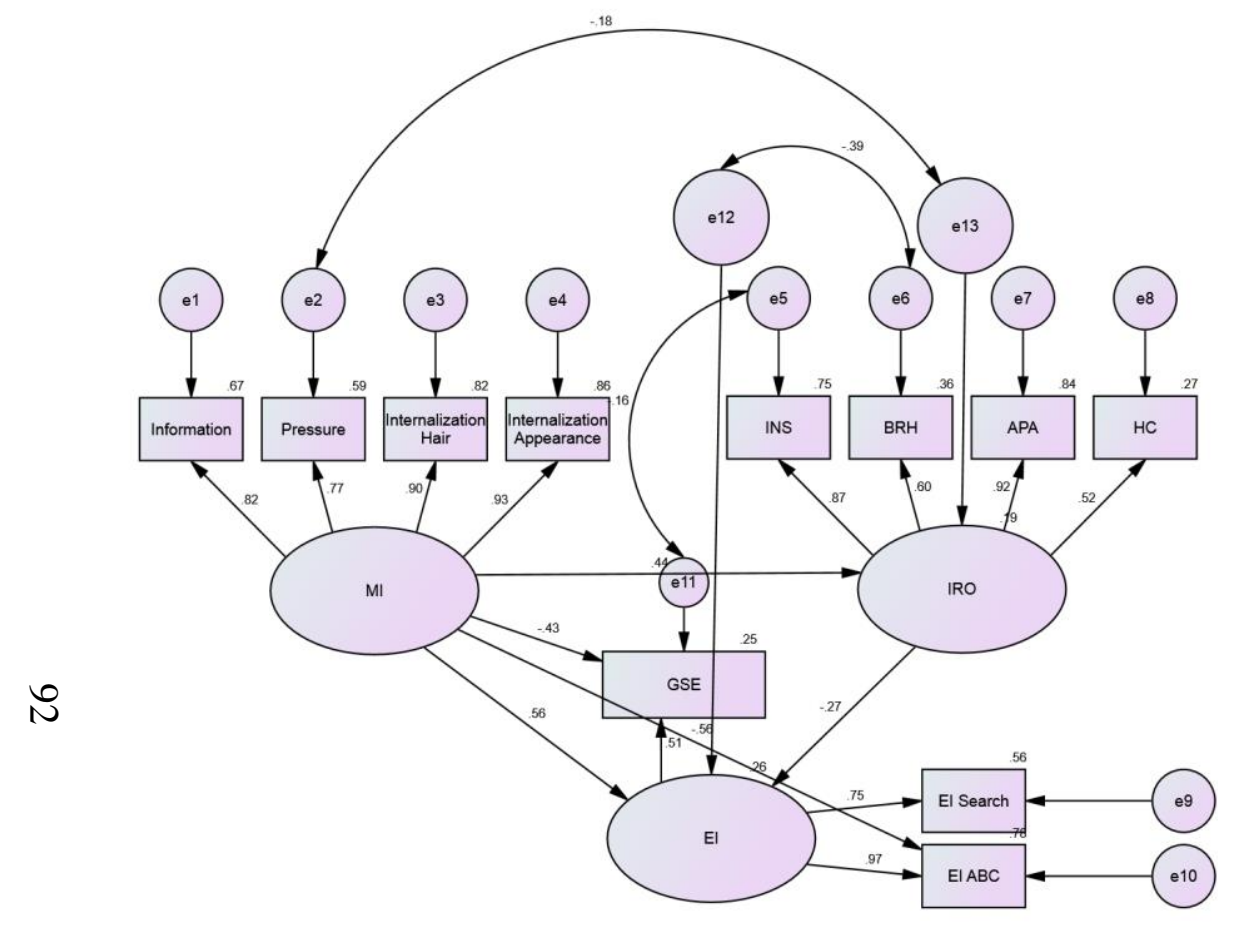

Figure 7. Respecified Model: Skin Tones 1 to 4.

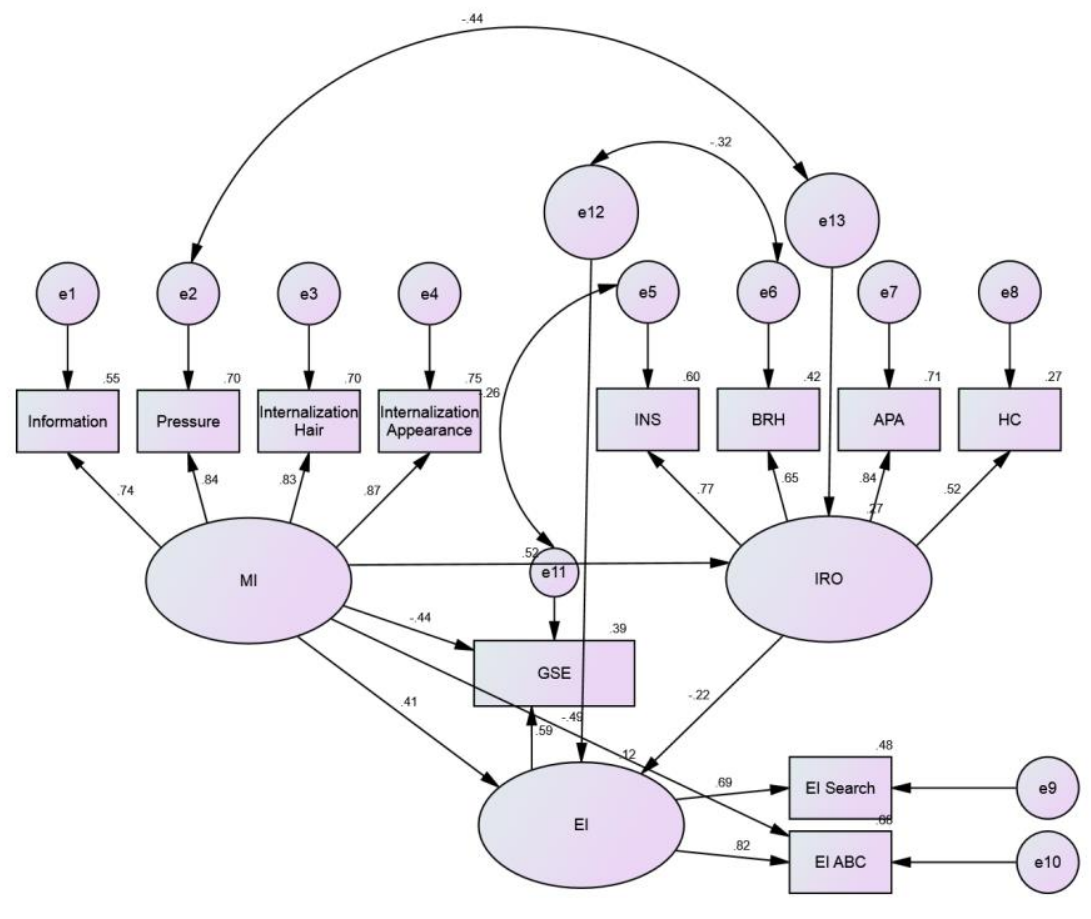

Figure 8. Respecified Model: Skin Tones 5 to 8. 


\section{CHAPTER 5}

\section{SUMMARY, DISCUSSION, AND IMPLICATIONS}

\section{Introduction}

This chapter will begin by summarizing the information contained in the previous four chapters. The purpose of this study will be outlined, the existing literature will be summarized, the methodology will be described, and the results will be presented. The main focus of this chapter will be devoted to a discussion of the findings. Limitations will be identified and implications for practice and future research will be explored.

\section{Purpose of the Study}

The first purpose of this study was to review the AA hair history and the current literature to highlight why hair issues and hair texture are important to the lives of AA and Black women. This purpose was achieved through the literature review, which will be summarized below. The second purpose of this study was to examine the impact of MI about hair texture on IRO, EI, and GSE through model testing and then determine if any differences existed within groups relating to ethnicity identification, hair texture, and skin tone. The fit of the model was described in the previous chapter and the implications of the results will be described below. The findings from this study contribute to the limited literature on MI, in particular the lack of literature on MI about hair texture and to the 
knowledge of the relationship between MI about hair texture and that impact on IRO, EI, and GSE of AA and Black women.

\section{Summary of the Literature Review}

In the United States racial hierarchies celebrate a single standard of beauty, a standard that devalues and excludes African hair textures (Arogundade, 2000; Gaskins, 1997; Taylor, 1999). Beauty has become so normalized that it no longer seems social, political, or racial in nature but "our entire cultural ethos in the contemporary United States seethes of white beauty" (Hunter, 2013, p. 63). Media images influence AA women to assume that there is one "ideal" and standard of beauty to which they must subscribe. This permeates the idea that AA women must assimilate to achieve because their own personal visage is not worthy, welcomed, or accepted. The majority of mass media reinforces racist conceptions by constructing historically distorted favorable images of Whites and negative images of people of color, in particular AAs (Allen, 2001). As a result of America's history of slavery and the effects of MI that contribute to continued oppression, EI and self-efficacy within the AA community is lost. The extent of the media's influence about hair texture as it relates to AA women's ethnicity, selfefficacy, and IRO is not known due to a lack of investigation on the topic. There is an absence of information on the specific role of MI and hair texture, and there is even less information on how MI about hair texture impacts IRO and self-efficacy of AA women. Little if any research has been based on the impact of the media portrayals of Caucasian American women on AA women as it relates to hair texture messages and influence. Meanwhile, more negative images of AA women than Caucasian American women continue to appear in the media (Frisby, 2004; Hill, 2002; Thomas, 2006). African 
American women are bombarded with negative images of themselves (Allen, 2001; Woodward \& Mastin, 2005) while being presented with an unattainable beauty standard that has gone unquestioned or challenged for decades. As a result trauma, internalized racism, and low self-esteem that greatly impacts all avenues of life have resulted unbeknownst to the AA woman and the AA community.

This damage to self that says Black beauty must be corrected to the mold of White beauty extends into black intelligence. Our country has a long history of portraying AAs as intellectually inferior, which may affect their participation in a field that focuses single-mindedly on the quality of one's intellect (Andrews, 2014). The link between perceived physical beauty as it relates to hair texture of AA women and personal selfefficacy is unknown. However, there is evidence to suggest that those (who navigate the world) who are classified as "beautiful" enjoy copious amounts of privilege. Those privileges range from academic success, job success, and in general more access that allows one entry to more and better resources to meet a desired end and ultimately achieve and succeed in unlimited areas of life. Eurocentric features result in more access, a less threatening presentation to White America, and is a means of survival for many who would normally due to stereotypes and biases are seen as unattractive and unlikable. Centuries of racism dictate white beauty standards, compelling Black females to rate their own natural hair texture within the framework of straight hair paradigms. "Racially motivated, concepts of good and bad hair are bad for Black females because they devalue hair textures most Black females have" (Robinson, 2011). The propagated influences of media messages as well as social experiences over long decades which have discriminated against darker skin and hair classified as bad have caused Black people to 
internalize the influential concept of good and bad hair amongst themselves and believe the messages broadcast that darker-skinned Blacks with kinkier hair are less attractive, less intelligent, and worth less than their lighter hued counterparts (Byrd \& Tharps, 2001). Beauty is power and many women have gained success, socially and otherwise banking on the marketability of their perceived physical attractiveness (Lakoff \& Scherr, 1984; Robinson \& Ward, 1995). Light skinned AAs still fare better economically, vocationally, and educationally (Wade, 1996). The impact of beauty on power and economic and social mobility leaves many AA women disenfranchised and disempowered because the definition of beauty is narrow and the standard of beauty by cultural and societal standards denies benefits, economic, social, education, vocation, and otherwise, to those who are not defined as such. The extent of this impact is the focus of this research.

\section{Methodology}

This study employed a nonexperimental research design using self-report surveys. The sample consisted of AA/Black female adults, 18 years or older, who were raised in the United States of America. Participants were individuals who signed up to complete surveys through QuestionPro, an online research platform; or whom voluntary elected to take the survey via email invitation and social media sharing of the online survey link.

Participant self-report quantified all variables. Participants completed surveys which measured their (a) MI about hair texture, (b) IRO, (c) EI, and (d) self-efficacy. Media influence about hair texture was measured with the SATAQ-3 Modified. Internalized racial oppression was measured with the IROS. Ethnic identity was measured with the MEIM. Self-efficacy was measured with the GSE. Structural equation 
modeling was used to test the model for the impact of MI about hair texture on IRO, EI, and self-efficacy.

\section{Findings and Discussion}

\section{Respondents’ Demographic Characteristics}

Respondents were drawn from a nonclinical sample, recruited through QuestionPro, a small literal arts university's population in Southwest Michigan, and through online social media, specifically Facebook. The participant sample included 322 adults who completed the full survey, were at least the age of 18 , female, raised in the United States of America, and identified as AA or Black. Respondents ranged in age from 18 to 75 and up. More than half of the sample, 52\%, identified ethnically as AA ( $N$ $=168), 37.5 \%$ identified as Black $(N=121), 6.2 \%$ identified as Biracial $(N=20)$, and 4.3\% identified as being Multiracial $(N=14)$.

Of the $N=322$ respondents, the reported hair types are as follows: $1=4.3 \%(N=$ $14) ; 2=3.7 \%(N=12) ; 3 \mathrm{a}=19.3 \%(N=17) ; 3 \mathrm{~b}=7.5 \%(N=24) ; 3 \mathrm{c}=24.2 \%(N=78)$; $4 \mathrm{a}=19.9 \%(N=64) ; 4 \mathrm{~b}=23.3 \%(N=75) ; 4 \mathrm{c}=11.8 \%(N=38)$. In addition, responses indicated that $16.8 \%$ of participants identified as having skin tone resembling photo $1(\mathrm{~N}$ $=54), 9.3 \%$ identified as having skin tone resembling photo $2(N=30), 9.6 \%$ identified as having skin tone resembling photo $3(N=31), 18 \%$ identified as having skin tone resembling photo $4(N=58), 22 \%$ identified as having skin tone resembling photo $5(N=$ 71), $7.1 \%$ identified as having skin tone resembling photo $6(N=23), 9.9 \%$ identified as having skin tone resembling photo $7(N=32)$, and $7.1 \%$ identified as having skin tone resembling photo $8(N=23)$. (Table 2; Appendix $\mathrm{C})$. 


\section{Zero-Order Correlations}

The correlations between MI about hair texture, IRO, EI, and GSE were in the moderate range in this study. In future studies results may be different, in that results may indicate lower or even higher correlations depending on the sample group. This means different sample groups may indicate lower or higher levels of media influence about hair texture, lower or higher levels of internalized racial oppression, lower or higher ethnic identity, and lower or higher levels of self-efficacy. Naturally, these will alter the results of future studies. However, this supports that these variables should continue to be studied as distinct constructs.

The significant correlations that existed between variables are theoretically consistent. Meaning, variables that were significantly corrected with MI were positively correlated (e.g., higher MI was correlated with higher IRO). There were also significant negative correlations among variables (e.g., higher MI was correlated with lower GSE).

\section{Research Question One and Model Fit}

\section{Is the hypothesized covariance matrix equal to the observed covariance matrix}

collected from the data? Structural equation modeling was used to examine whether the hypothesized relationships among variables in the conceptual model were supported in this sample. Structural equation modeling indicated that the original hypothesized model was a poor fit to the data $(\mathrm{GFI}=.83 ; \mathrm{NFI}=.78 ; \mathrm{CFI}=.82 ; \mathrm{RMSEA}=.14)$, so revisions were made on the basis of both theory and modification indices. The respecification included one added path between latent variables (IRO to EI) and two paths between latent variables and observed variable (EI to GSE and MI to EI ABC) that resulted in an excellent fit for the data $(\mathrm{GFI}=.96 ; \mathrm{NFI}=.96 ; \mathrm{CFI}=.98 ; \mathrm{RMSEA}=.05)$. 


\section{Media Influence About Hair Texture and Internalized Racial Oppression}

The path from MI to IRO indicated a moderate effect $(r=.47)$ with statistical significance $(p<.001)$. As predicted, these findings indicate that MI about hair texture influence IRO. MI both directly and indirectly influences both EI and GSE through IRO. The statistical correlations are positive, which suggest that higher levels of MI about hair texture, increase IRO, which indirectly affects GSE. These findings agree with research that suggests greater levels of MI about hair texture can produce IRO, even unconsciously, within viewers of these propagated media messages (Byrd \& Tharps, 2001; Woodson, 2002). Of the four observed variables, shown in Table 5, (Information, Pressure, Internalization-Hair, and Internalization-Appearance) represented by the latent variable MI, shown in Figure 2, respondents MIs about hair texture within their sociocultural content were all high, over $53 \%$ across the board, with the highest forms of MI being obtained from informational sources at $58 \%$ and the internalization of hair influences at 58\%. Of the four observed variables (INS, BRH, APA, and HC) represented by the latent variable IRO, respondents averaged a score of $55 \%$ on the subscale BRH indicative of one's belief in the bias representation of history; and an average of $62 \%$ on the subscale $\mathrm{HC}$ indicative of one's desire to change their natural hair texture. Meaning respondents were most affected and had higher levels of MI about hair texture and IRO based on these factors.

The effects of media messages and their influence have continued to keep AA women in chains by the portrayal of European women coined as the beauty standard and the absence of unaltered AA women in media images. And a group simply cannot walk 
away from such systematic assaults unscathed, particularity if the assault continues in new ways, which is the modern day struggle (Wyatt, 1977). The media is a profound source of cultural teaching with an unmatched power to reach millions quickly and conveniently and has been used as a tool of oppression (White, 2008) in which it creates an unconscious prejudice through visual imaging directed at AA women. The lack of visibility of AA women and the stereotypical manner in which they are represented greatly limit one's conception of themselves and ideas about what it means to be beautiful and accepted (White, 2008). African American women who rarely experience the full range of images that truly represent them in diversity of skin tones, hair textures, shape and size will begin to think that who they are is not acceptable and definitely not what's “in” (White, 2008). When in fact AAs are represented, content analyses reveal that the majority of media depictions of AA women (in Black-oriented media and mass media) are those with light skin, straight hair, and small noses and lips (Capodilupo, 2010).

\section{Media Influence About Hair Texture and Ethnic Identity}

The path from MI to EI indicated a large effect $(r=.51)$ with statistical significance $(p<.05)$. These finding indicate that MI about hair texture influence EI. MI about hair texture has both a direct and nondirect effect on EI. What's noninutuive is that the correlations are positive, meaning in this sample study, greater levels of MI about hair texture seemed to increase EI. This is nonintuitive as research suggests that due to the wide consumption of dominant culture media by AAs (Riggott, 2013; Ward, 2004), AA women tend to internalize the negative images they see (Allen, 2001; Fujioka, 2005; 
White, 2008). However, it is impossible to know how every form of media within the various platforms effect each individual or how individuals perceive, understand, and respond to the influential images they see. This is true in the sense that research has also suggested that EI might still be increased from other images within the media such as prominent AA and Black athletes and entertainers that would increase one's desire to ethnically identify with their group and solidify membership to that group (Chambers et al., 1994; Wade, 1996).

African American and Black women receive mixed messages from the media and so one can have a high general sense of EI, as demonstrated from the results of this present study, but still suffer from IRO. This includes the acceptance of only certain members of one's group, such as those with mixed hair textures that are deemed beautiful; or light skin versus dark skin AAs. There are also AA and Black individuals who are more intentional in investing in the types of media they choose to partake of and may intentionally seek out media sources and platforms that highlight the AA and Black people in a positive and fair light including their successes, accomplishments, and achievements.

\section{Media Influence About Hair Texture and Ethnic Identity Affirmation, Belonging, and Commitment}

The path from MI to EI ABC indicated a large effect $(r=-.54)$, however no statistical significance was shown from this sample. These findings indicate that MI about hair texture influence EI both positively as previously discussed and negatively as currently revealed. The statistical correlations on EI in this case are negative as they pertain to $\mathrm{EI} A \mathrm{BC}$. These findings agree with research that suggests greater levels of $\mathrm{MI}$ 
about hair texture decrease $\mathrm{EI} \mathrm{ABC}$, which are specific to the areas of one's affirmation, belonging, and commitment to their EI. There seems to be two conflicting results where MI about hair texture can both increase and decrease one's level of EI. However, AA and Black women receive mixed messages from the media and so one can have a high general sense of EI, as demonstrated from the results of this present study, but still suffer from IRO which can influence their EI pertaining to how close and inclusively apart of individuals feel to their ethnic group. This also demonstrates the intercorrelational and connected relationship between the examined variables.

\section{Media Influence About Hair Texture and Self-Efficacy}

The path from MI to GSE indicated a moderate effect $(r=-.45)$ with statistical significance $(p<.001)$. These findings indicate that MI about hair texture influence GSE both directly and indirectly though IRO and EI. The statistical correlations are negative, which suggests that higher levels of MI about hair texture decrease GSE. These findings agree with research that suggests greater levels of MI about hair texture as a minority in majority culture are damaging to the AA women's self-esteem and produces unconscious IRO (Green et al., 2000; Kaw, 1994; Lakoff \& Scherr, 1984) and the idea that one can only obtain success through conforming to the majority beauty standards (Allen, 2016; Dossou, 2013; Rhodan, 2014), which as a result has become a means of survival for many AA women as one's ability to successfully and strategically navigate the world depends largely on how they are perceived and a great deal of that is hinged upon how we look. Dress codes in schools that target AA by forbidding Black hair styles and grooming regulations within the military and corporate world suggests to Black women 
that only through changing physical features will persons' of African descent be afforded privilege, class mobility, and social acceptance by the dominant culture (Rooks, 1996).

\section{Internalized Racial Oppression and Ethnic Identity}

The path from IRO to EI indicated a small effect $(r=-.25)$ with statistical significance $(p<.001)$. These findings indicate that IRO influences EI both directly and indirectly through MI about hair texture. The statistical correlations are negative, which suggests that higher levels of IRO decrease EI. These findings agree with research that suggests an increase in IRO may cause a decrease in EI due to the adoption of racist messages about one's ethnic group causing one to be less inclined to ethnically identify as a member of their ethnic group. This in turn would result in a decrease in belonging and less overall involvement and engagement with one's group drastically decreasing EI achievement (Hall, 1986; Phinney, 1992; Pike, 2010).

\section{Ethnic Identity and Self-Efficacy}

The path from EI to GSE indicated a large effect $(r=.55)$ with statistical significance $(p<.001)$. These findings indicate EI influences GSE both directly and indirectly through IRO. The statistical correlations are positive, which suggests that higher levels of EI increase GSE. These findings agree with research that suggests positive EI has been linked to aspects of self-esteem, confidence, positive selfadjustment, and subjective well-being, and reduced depressive symptoms, which are all indicators of one's overall abilities to cope with daily life and achieve intended results (Ormrod, 2006; Yap et al., 2014). Ethnic identity is considered a critical component of the self-concept and crucial to the psychological well-being of members of an ethnic 
group and plays a major role in how a person approaches goals, tasks, challenges, and life (Luszcynska \& Schwarzer, 2005).

\section{Research Question Two and Fitted Model}

Does ethnic identification, hair texture, or skin tone influence the fit of the empirical model? After the modifications discussed above, the resulting model was then used to evaluate whether differences within the sample group existed pertaining to ethnic identification, hair texture, and skin tone. The observed data within the between groups all indicated good model fits with several significant differences.

\section{Ethnic Identification}

As previously discussed, AA and Black are used interchangeably within society. However, Black is the overarching term, which is inclusive of AAs. Black often pertains to individuals who identify with having African heritage however may not identify as being American because they claim another country of origin as their home; but this does not mean that they were not raised fully or even partially in America therefore being influenced by American society. In this respect, and for the purpose of this study, Black is not a color or a skin tone but a particular ethnicity which includes the AfricanAmerican and Black experience because of the particular ancestry and associated combined physical, facial, skin, and hair features.

In comparing the two groups, AA and Black, the data in Table 8 indicated several significant differences between the groups as it pertains to AA and Black ethnically identified women. First, MI and IRO was more strongly correlated among AA women ( $r$ $=.53)$ than black women $(r=.42)$. Certain Black women may in part experience lower 
levels of IRO, as in this sample group, because they see themselves as being immune from certain aspects of American culture due to identification and connection with another country origin in which they are the majority or they may be more attune, as some AAs are, to the nature of American cultural influences and have found a way to shield themselves from various negative images that contribute to IRO.

Second, MI and EI was more strongly correlated among Black women $(r=.66)$ than AA women $(r=.44)$. As previously discussed, AA and Black women receive mixed messages from the media and so one can have a high general sense of EI, as demonstrated from the results of this present study, but still suffer from IRO. Black women may fare slightly better in maintaining aspects of their EI due to a variety of different factors including not identifying as American, where they fall on the skin tone and hair texture continuum, available coping resources, and perception and consumption of MIs. Furthermore, there is the argument that how people perceive ethnicity, both their own and that of others, can be tough to measure, due to subjectivity (Fisher, 2013).

Third, EI and GSE was more strongly correlated among AA women $(r=.75)$ than Black women $(r=.34)$. AA women tend to internalize the negative images they see (Allen, 2001; Fujioka, 2005; White, 2008) and are at a higher risk of damage to their selfconception due to the prejudice in American society that permeates every orifice of their lives. However, when one is able to in various ways maintain positive EI they in turn are also able to bolster self-efficacy as positive EI has been linked to aspects of positive adjustment, self-esteem, subjective well-being, and reduced depressive symptoms (Yap et al., 2014). 
Fourth, IRO does not explain a decreases in EI within the AA model ( $r=-.04)$ but it does within the Black model $(r=-.53)$. If Black women are able to maintain higher levels of EI despite MI about hair texture, as indicated within this study, it stands to reason that when they are in fact infiltrated by the media's influence causing IRO, this indirect effect on their EI is a crushing blow. This finding is also indicative of the intercorrelational aspects involved pertaining to these variables and how the intersectionality of each plays a role on another, which can significantly impact the lives of minority women. For AA women, this may also be indicative of the subjective nature of ethnic identity and the argument that it is difficult to actually measure (Fisher, 2013), despite good faith attempts.

\section{Hair Texture}

In comparing the two groups, hair textures 1 to 4 and 5 to 8 , the data in Table 8 indicated two significant differences between the groups as it pertains to hair textures. First, MI and IRO was more strongly correlated among hair textures 1 to $4(r=.62)$ than textures 5 to $8(r=.43)$. Though this correlation is positive, meaning higher levels of MI about hair textures increase IRO, it is nonintuitive for this particular hair texture grouping. Hair textures 1 to 4 are those which were less like the minority, however when looking at the demographic characteristics of this sample in Table 2, the majority of the respondents, about $13 \%$, identified as having hair textures $3 \mathrm{a}$ and $3 \mathrm{~b}$, which are still hair textures that are not as smooth or straight, though less coily than hair textures in group 5 to 8. Depending on the precise nature of these identified women's particular hair texture in this group and where they fall on the hair texture and skin color continuum, they may 
still experience hair discrimination in varying degrees among other negative media messages about their hair textures resulting in IRO.

Second, MI and EI was more strongly correlated among hair textures 1 to 4 ( $r=$ $.63)$ than textures 5 to $8(r=.48)$. Again, hair types and textures are on a continuum that widely various in differing degrees based on multiple factors. In this case, those who possessed hair most closely resembling the majority were better able to maintain aspects of their EI.

\section{Skin Tone}

In comparing the two groups, skin tones 1 to 4 and 5 to 8 , the data in Table 8 indicated two significant differences between the groups as it pertains to skin tones. First, MI and EI was more strongly correlated among skin tones 1 to $4(r=.56)$ than skin tones 5 to $8(r=.41)$. Research has shown that AAs who have lighter skin tend to identify less strongly with Black ethnicity, and more with middle-class white cultures and values (Hughes \& Hertel, 1990).

Second, EI and GSE was more strongly correlated among skin tones 5 to $8(r=$ .59) skin tones 1 to $4(r=.51)$. Skin tones 5 to 8 are those which are darker. And as previously discussed, AA women tend to internalize the negative images they see (Allen, 2001; Fujioka, 2005; White, 2008) and are at a higher risk of damage to their selfconception due to the prejudice in American society that permeates every orifice of their lives. However, when one is able to in various ways maintain positive EI they in turn are also able to bolster self-efficacy as positive EI has been linked to aspects of positive adjustment, self-esteem, subjective well-being, and reduced depressive symptoms (Yap et 
al., 2014). However, light skinned AA still fare better economically, vocationally, and educationally (Wade, 1996).

\section{Implications of the Study}

The effects of MI are widespread and far-reaching. There is no crevice the media cannot touch or influence, for better or for worse. Media influence about hair texture, in this current study, were positively correlated with high levels of IRO; negatively correlated with low levels of EI as it pertains to affirmation, commitment, and belonging; and negatively correlated with low levels of self-efficacy among AA and Black women.

This is an important finding considering AAs and Blacks are the minority in numbers and representation but the majority of media users and consumers. African Americans are the largest consumers of media in a Eurocentric American culture that propagates and bolsters white beauty, silky smooth hair textures, and light skin. It comes as no surprise, considering these facts that IRO levels are high among AA women, and EI and GSE are low. This is not to say that all AA and Black women are affected, but there are enough that are to which questions should arise and the effects of MI about hair texture be looked at to gain understanding of this phenomenon and taken seriously. Aspects of skin tone, mixed with hair texture affect everything from life expectancy and mate selection (Hughes \& Hertel, 1990; Hunter, 1998) to prison sentencing (Hochschild, 2007) and identity (Brown, 1998; Thompson \& Keith, 2004). Colorism and hair texture translates into real socio-economic differences (Hill, 1999) among both dark and light skinned Blacks (Herring, 2004).

Educators and other professionals need to better understand these dynamics to aid clients and students who may be dealing with shame associated with their hair texture, 
IRO, and low GSE due to societal impacts that promote these feelings within AA and Black women. Furthermore, society on a whole, in particular the majority that propagate majority culture and Eurocentric beauty standards need to engage in direct and intentional diversity inclusion efforts to afford ethnic minorities, in particular AA and Black women the same media representation it affords to white women.

The Eurocentric standard of beauty that American culture and society incessantly promotes is on its face damaging to AA and Black females, no matter their age, as it does not reflect back Black beauty. One's ability to successfully and strategically navigate the world depends largely on how they are perceived and a great deal of that is hinged upon how one looks. Beauty is power and survival. Hair is no different from a nose, or lips, or skin color. Hair is a defining feature and what shape or texture it takes impacts how we see the world and how the world sees us.

Hair like skin tone or complexion will never be "blind" because society sees it even if they choose not to acknowledge it outside the covert ways in which it accepts the majority and rejects the minority. Society sees hair. Society sees hair shape, hair color, hair texture, hair density, and hair length. What is seen is then placed in some context in connection with all other attributes of physical appearance to form an opinion and make an assessment of who an individual is, what category one belongs to, and this leads to decisions on how then one should be treated and addressed. The minority by majority vote are then overtly or covertly told what they can and cannot achieve and are unanimously and arbitrarily prescribed limits and have heights capped based upon hair texture and all other associated features relating to Blackness. 
Representation matters. Even more, fair, accurate, and continuous representation matters for AA and Black women in the media pertaining to hair texture. The majority culture Eurocentric beauty standard has permeated the societal atmosphere of media for decades. Its value system is so insidiously propagated that the effects go unbeknownst to those affected. The minority begins to value the majority due to no fault of their own. But when the minority tends to value the majority culture, in turn they devalue their own selves. Representation matters and is important because if the minority only see esteem, worth, goodness, and virtue in the majority culture standards of beauty, emanated through the lens, viewing, and portrayal of White women that begins to negatively transform into every aspect of Black life.

Representation is powerful. The movie Black Panther allowed AAs to positively ethnically identify merely by seeing themselves in a positive light in which the African people are rich in culture, heritage, resources, and technology. The women were intelligent as both fierce warriors and creators of advanced technology while beautiful in their natural hair textures (or with no hair at all) and melanated (dark) skin tones. One movie empowered the AA community in a powerful, significant, and meaningful way. Imagine how continuous images, storylines, and MIs like these could bolster EI and GSE and decrease, or even eradicate IRO. It can happen, but first American culture and the majority must see and care about the effects of MI about hair texture on the IRO, EI, and GSE of AA and Black women. Care enough to do something about it.

\section{Limitations}

Several limitations should be considered with this study. First, this study is intended to be informative about how the media can influence AA women's feelings 
about their hair texture and thereby affecting their EI, GSE, and IRO; however, the current study was nonexperimental in design. This means that there can be no direct cause-and-effect statements regarding statistical results. However, for this current study, a nonexperimental design was appropriate as I sought to measure variables as they were experienced by the respondents'. The model tested was based on the literature review outlined in Chapter 2. The literature detailed was a historic and experiential review of AA history and the Black experience on captured in part as it pertains to issues of hair texture and beauty.

Second, all variables were assessed using self-reported measures. In addition, participants were made aware of what the study was aiming to evaluate and may have therefore resulted in participants answering questions in a socially desirable way versus in a manner that reflected their true beliefs, feelings, knowledge, or experiences. However, the nature of the variables made self-reported measures necessary in order to gather individualized subjective information.

Third, my survey was conducted online, limiting my sample to individuals with internet and computer assess, who were technologically savvy, and who had membership to QuestionPro, a small literal arts university in Southwest Michigan, or Facebook.

Lastly, I analyzed my data using SEM. Therefore, my results speak to the degree of fit observed between my resulting SEM model and the data from the current sample. As previously mentioned, it may be likely that different models (using different variables or even the same variables arranged in a different configuration) from different sample groups will render different results, meaning the model could be better fitted to the data or even poorly fitted. Structural equation modeling was a good approach for my research 
questions because it allowed me to assess multiple variables, both observed and unobserved, simultaneously.

\section{Recommendations}

Hair texture isn't seen as an important research topic because overall people just don't understand its importance or significance, especially when one isn't personally affected by it. Black hair is even lower on the totem pole of being noteworthy of any serious research attention. And yet, black hair has serious ramifications for the everyday lives of Black women. Black women are affected daily by a society that looks less like their reflection in the mirror than it resembles it. Until this point, research and practice has been extremely limited by lack of data based knowledge as it pertains to MI about hair texture on AA and Black women, in particular, IRO, EI, and GSE. Therefore my model of the relationship between MI about hair texture, IRO, EI, and GSE makes an important contribution to advancing understanding in the field.

It is important for researchers, educators, mental health professionals, and lay people to be better understand hair dynamics of AA and Black women so they will be better able to serve, represent, appreciate, and appropriately interact with each individual they encounter in a fair and unbiased way. Understanding and diversity education is needed to bridge the gap and to create effective and meaningful change for good in which AA and Black women's natural hair textures are not obliterated in media, the classroom, work spaces, or armed forces, but included. 


\section{Recommendations for Practice}

1. Educators and professionals need to intentionally engage in diversity, cultural, and sensitivity training to address and recognize one's own biases and stereotypes about matters related to AA women's hair.

2. Diversity, cultural, and sensitivity trainings need to be established within workplaces and institutions that are majority cultured to both become educated on matters as it pertains to AA women's hair and begin the dialogue needed to reframe westernized views of what is neat, acceptable, and professional because the current limited worldviews are affecting Black women's ability to effectively navigate through everyday life, work, serve in the military, and learn within the classroom setting.

3. Educators and professionals need to engage in social justice advocacy that empowers Black women and petitions to change bylaws within schools, institutions, and corporate America that are discriminatory and punitive toward AA women for wearing their natural hair.

4. Laws of protection need to be enacted in which AA women's natural hair and associated hairstyles are protected against discriminatory practices that prevent them from functioning within society, as it pertains to employment and educational settings to prevent AA women from being stigmatized as unprofessional in the workplace, rebellious within society, or a distraction in the classroom because of their natural hair.

5. The media needs to be better managed in which checks and balances are put into play concerning what is aired, published, and presented so that there is equal 
representation of AA women, in particular, their hair textures and hair styles because media influences can be damaging to women of color by increasing levels of internalized racism and lowering self-efficacy.

\section{Future Research}

1. Future research should examine how MI about hair texture affects other areas of Black women's lives such as workplace studies, military studies, and in depth educational studies.

2. Future research should examine the difference within Historically Black Colleges and Universities vs. Predominantly White Institutions and the effects of MI about hair texture on IRO, EI, and self-efficacy of AA women; with additional components such as self-esteem and disorders such as anxiety and depression.

3. Future research should investigate the differences between age groups, both young and old, given the burst of media usage over the past decade to determine if younger generations, including children, are more influenced by media than older generations; or are older generations just as impacted due to living the trauma of the slavery narrative personally or passed down through generational trauma.

4. Future research should utilize qualitative methods to collect narrative from the participants to gain a greater depth of understanding from AA women's lived experiences and how those experiences have impacted their lives as it pertains to MI about hair texture as well as skin tone.

5. Future research should investigate the impact of MI on AA women as it pertains to self-esteem, body image, body size, weight, and femininity as this also is within the realm of beauty standards heavily propagated within majority 
American culture, which dictates what size a woman should be and how her body should look leaving AA women, who usually have higher Body Mass Indexes and larger body types as "othered" in body image and femininity.

6. Future research should continue to investigate the various aspects pertaining to the MEIM and how to gauge and measure the numerous facets of ethnic identity. 
APPENDIX A

APPROVAL LETTERS 


\title{
Andrews 1 University
}

December 13, 2017

Kristy La Mar

Tel: 516-385-8352

Email: kristy@andrews.edu

\begin{abstract}
RE: APPLICATION FOR APPROVAL OF RESEARCH INVOLVING HUMAN SUBJECTS IRB Protocol \#: 17-178 Application Type: Original Dept.: Graduate Psychology \& Counseling Review Category: Expedited Action Taken: Approved Advisor: Carole Woolford-Hunt Title: The impact of media influence on feelings about hair texture on internalized racial oppression, ethnic identity, and self-efficacy.
\end{abstract}

This letter is to advise you that the Institutional Review Board (IRB) has reviewed and approved your IRB application for research involving human subjects entitled: "The impact of media influence on feelings about hair texture on internalized racial oppression, ethnic identity, and self-efficacy" IRB protocol number 17-178 under Expedited category. This approval is valid until December 13, 2018. If your research is not completed by the end of this period you must apply for an extension at least four weeks prior to the expiration date. We ask that you inform IRB whenever you complete your research. Please reference the protocol number in future correspondence regarding this study.

Any future changes (see IRB Handbook pages 10-11) made to the study design and/or consent form require prior approval from the IRB before such changes can be implemented. Please use the attached report form to request for modifications, extension and completion of your study.

While there appears to be no more than minimum risk with your study, should an incidence occur that results in a research-related adverse reaction and/or physical injury, (see IRB Handbook page 11) this must be reported immediately in writing to the IRB. Any projectrelated physical injury must also be reported immediately to the University physician, Dr. Katherine, by calling (269) 473-2222. Please feel free to contact our office if you have questions.

Best wishes in your research.

Sincerely

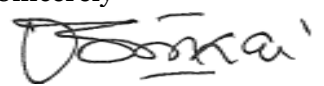

Mordekai Ongo

Research Integrity \& Compliance Officer

Institutional Review Board - 8488 E Campus Circle Dr Room 234 - Berrien Springs, MI 49104-0355

Tel: (269) 471-6361 Fax: (269) 471-6543 E-mail: irb@andrews.edu 
APPENDIX B

INFORMED CONSENT 


\section{Informed Consent}

Purpose: You are invited to participate in a research project titled: "The Impact of Media Influence about Hair Texture on Internalized Racial Oppression, Ethnic Identity, and SelfEfficacy." The purpose of this research project is to examine the relationship between media influence about hair texture, internalized racial oppression, ethnic identity, and selfefficacy.

Researchers: This research is being conducted by Kristy L. La Mar, a PhD student in the department of Graduate Psychology and Counseling at Andrews University in Berrien Springs, Michigan. The research is being supervised by Dr. Carole Woolford-Hunt, PhD. Results from the research will be used in Kristy L. La Mar's dissertation and may be published in professional literature or presented at conferences.

Procedure: If you choose to participate in this research, you will be asked to complete a series of surveys about yourself pertaining to your feelings about yourself and questions about your daily life. The survey will take approximately 30 minutes to complete.

Participation: In order to participate, you must be 18 years of age and over, female, and identity as African American. Your participation in this study is completely voluntary. It is your choice whether to participate or not. You may discontinue the survey at any time.

Risks/Benefits: There is no foreseeable risks or benefits associated with participation in this research. However, you will contribute to increasing and needed empirical research on this subject matter.

Confidentiality: Your survey responses will be strictly confidential and data from this research will be reported only in the aggregate. Your information will be coded and will remain confidential.

Contact Information: If you have questions at any time about the survey, your participation in this research, or your rights as a participant, you may contact the principle investigator, Kristy L. La Mar at kristy@ andrews.edu. You may also contact the research advisor Dr. Carole Woolford-Hunt at (269) 471-3473 or cwh@andrews.edu.

Consent: Thank you very much for your time and participation. Please start the survey by clicking on the Continue button below. By clicking this button, you are giving your consent to participate in the research described above. 
APPENDIX C

DEMOGRAPHIC \& HAIR QUESTIONNAIRES 


\section{DEMOGRAPHIC QUESTIONNAIRE}

1. Age: What is your age?

- $18-24$

- $25-34$

- $35-44$

- $45-54$

- 55-64

- $65-74$

- $75+$

2. Ethnicity: Please specify your ethnicity.

- African American

- Black

- Biracial

- Native American or American Indian

- Hispanic or Latino

- Asian or Pacific Islander

- White

- Other

3. Education: What is the highest degree or level of school you have completed? If currently enrolled, highest degree received.

- No schooling completed

- Nursery school to $8^{\text {th }}$ grade

- Some high school, no diploma

- High school graduate, diploma or equivalent (for example: GED)

- Some college credit, no degree

- Trade/technical/vocational training

- Associate degree

- Bachelor's degree

- Master's degree

- Professional degree

- Doctorate degree

4. Marital Status: What is your marital status?

- Single, never married

- Committed relationship

- Married or domestic partnership

- Widowed 
- Divorced

- Separated

5. Employment Status: Are you currently...?

- Employed for wages

- Self-employed

- Out of work and looking for work

- Out of work but not currently looking for work

- A homemaker

- A student

- Military

- Retired

- Unable to work

6. Where were you born?

7. Where were you raised? 


\section{HAIR TEXTURE QUESTIONAIRE}

1. How do you feel about your natural hair (unprocessed/ unaltered)?

- Very satisfied

- Satisfied

- Neither satisfied nor dissatisfied

- Dissatisfied

- Very dissatisfied

2. Have you ever changed your hair because of something/someone you saw/read about in the media? [Select all that apply]

- No

- Changed my hairstyle

- Changed my hair length

- Changed my hair texture

- Changed my hair color

- Purchased a wig/ weave

- Other (please explain)

3. What hair typing most describes your natural hair texture (unprocessed/ unaltered)?

- 1

- 2

- $3 \mathrm{a}$

- $3 b$

- $3 \mathrm{c}$

- $4 \mathrm{a}$

- $4 \mathrm{~b}$

- $4 \mathrm{c}$

- I don’t know

4. I feel more confident wearing a wig/ weave

- No

- Yes

5. I feel happy with my natural hair texture (unprocessed/ unaltered)

- No

- Yes

6. I feel happy with my natural hair length (unprocessed/ unaltered)

- No 
- Yes

7. I feel more beautiful wearing a wig /weave with a smoother or longer hair texture

- No

- Yes

8. I wish my natural hair were of a better texture or longer in length

- No

- Yes

9. I would describe my natural hair as "good hair"

- No

- Yes

10. I would describe my natural hair as "bad hair"

- No

- Yes

11. Which does your natural hair texture (unprocessed/ unaltered) look most similar to (from image below)

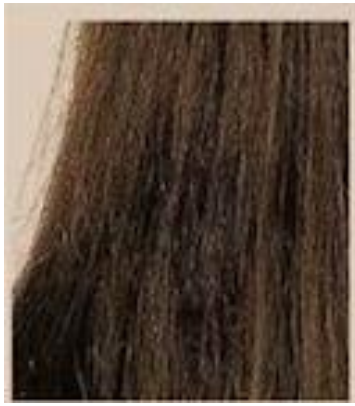

1

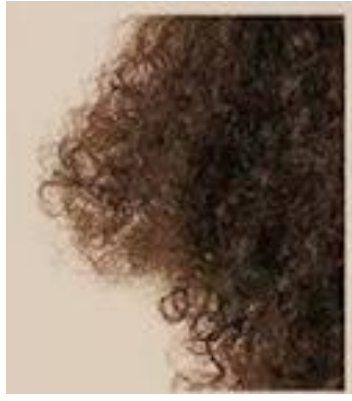

$3 \mathrm{c}$

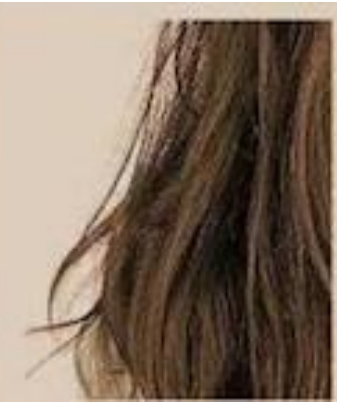

2

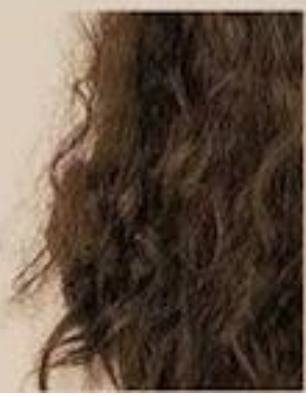

$3 a$

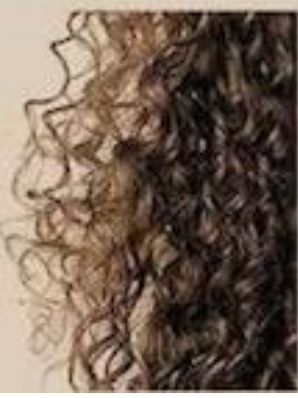

$3 b$

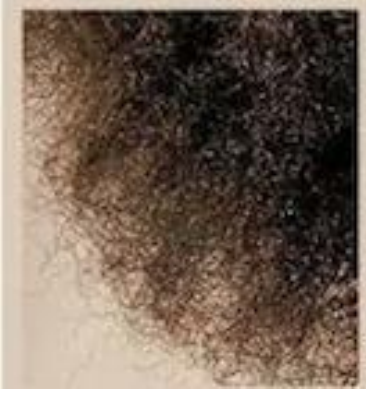

$4 a$

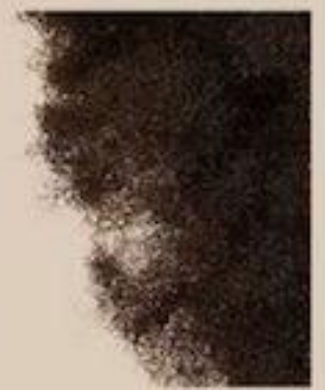

$4 b$

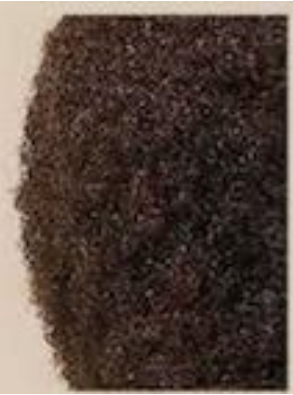

$4 c$ 
12. The hair texture I most prefer (Select one from the image below)
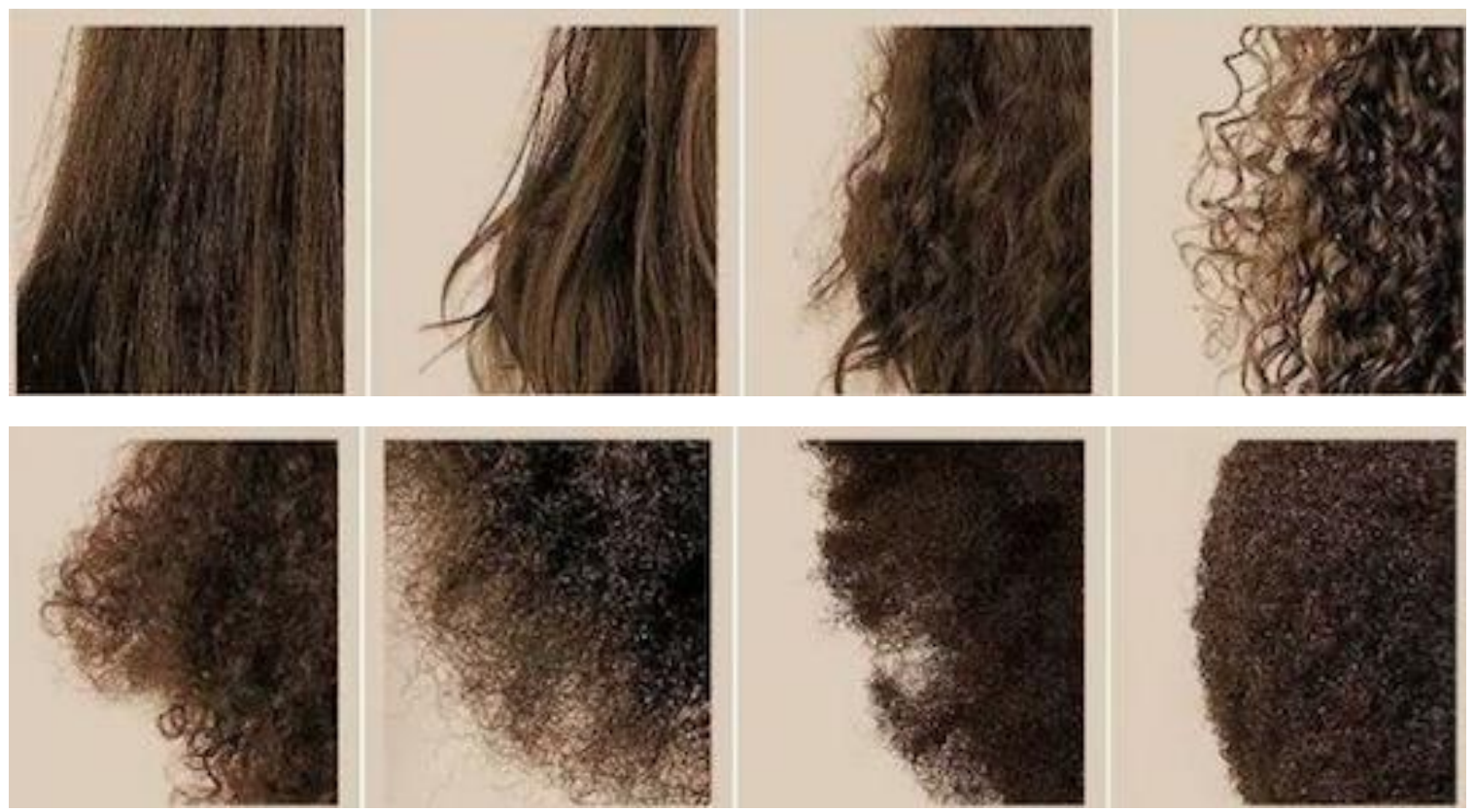

13. My skin tone most resembles (Select one from the photo below)

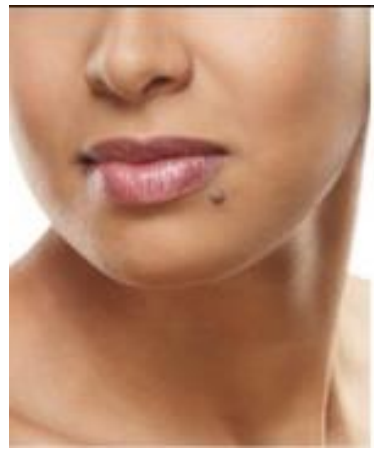

1

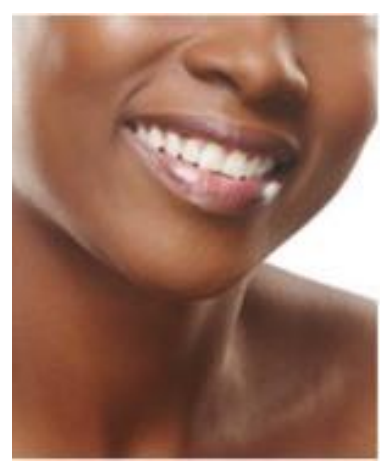

5

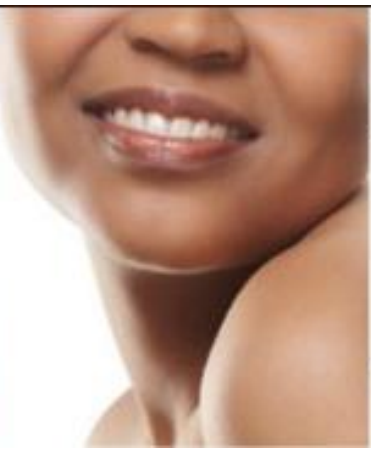

2

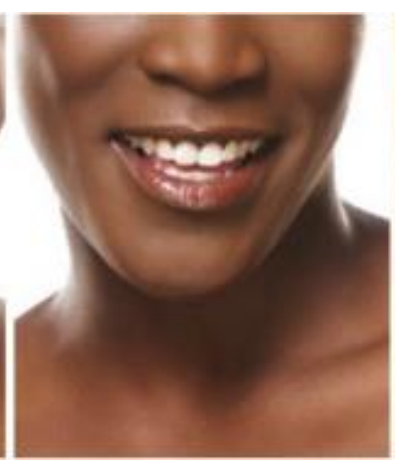

6

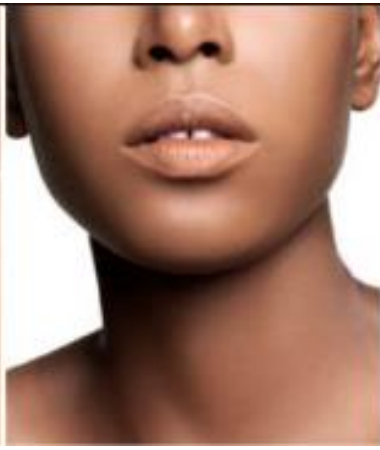

3

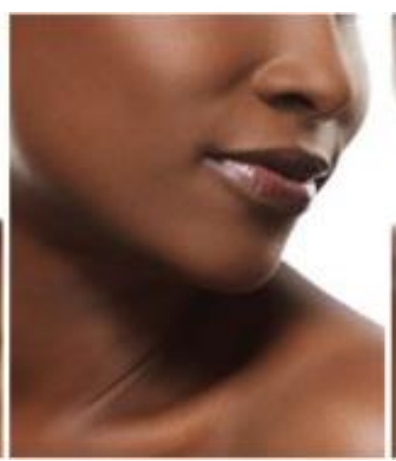

7

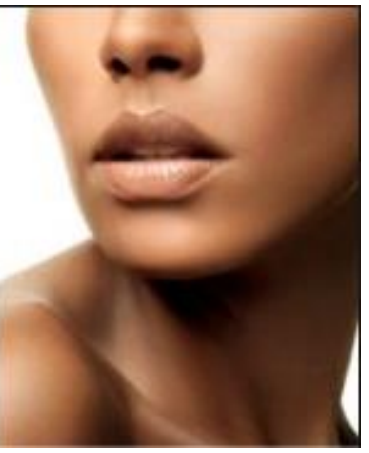

4

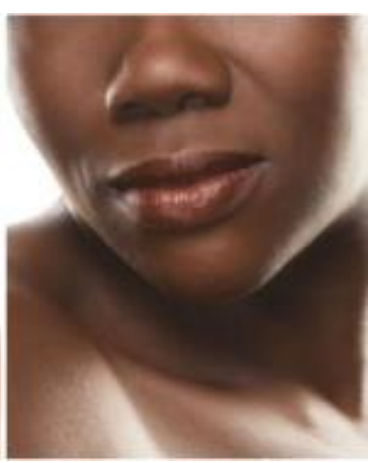

8 
14. The skin tone I most prefer (Select one from the photo below)

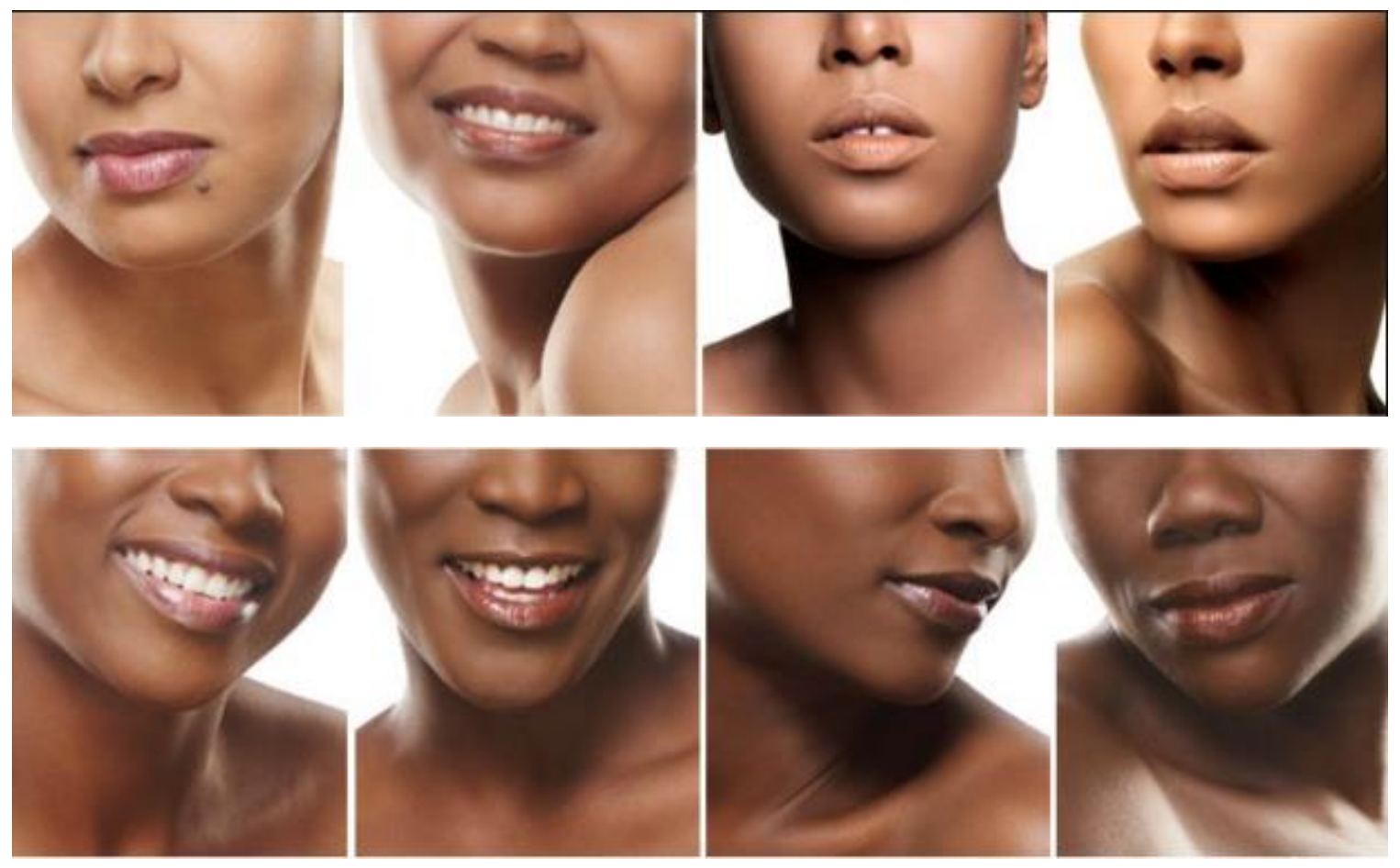


APPENDIX D

SURVEYS 


\section{Sociocultural Attitudes Toward Appearance Questionnaire-3 Modified}

Please read each of the following items carefully and indicate the number that best reflects your agreement with the statement.

$$
\begin{aligned}
& 1=\text { Definitely Disagree } \\
& 2=\text { Mostly Disagree } \\
& 3=\text { Neither Agree Nor Disagree } \\
& 4=\text { Mostly Agree } \\
& 5=\text { Definitely Agree }
\end{aligned}
$$

\begin{tabular}{|c|c|c|c|c|c|}
\hline $\begin{array}{l}\text { 1. TV programs are an important source } \\
\text { of information about hair and "being } \\
\text { attractive." }\end{array}$ & 1 & 2 & 3 & 4 & 5 \\
\hline $\begin{array}{l}\text { 2. I've felt pressure from TV or } \\
\text { magazines to have longer hair. }\end{array}$ & 1 & 2 & 3 & 4 & 5 \\
\hline $\begin{array}{l}\text { 3. I do not care if my hair looks like the } \\
\text { hair of people who are on TV. }\end{array}$ & 1 & 2 & 3 & 4 & 5 \\
\hline $\begin{array}{l}\text { 4. I compare my hair to the hair textures } \\
\text { and styles of people who are on TV. }\end{array}$ & 1 & 2 & 3 & 4 & 5 \\
\hline $\begin{array}{l}\text { 5. TV commercials are an important } \\
\text { source of information about hair and "being } \\
\text { attractive." }\end{array}$ & 1 & 2 & 3 & 4 & 5 \\
\hline $\begin{array}{l}\text { 6. I do not feel pressure from TV or } \\
\text { magazines to look pretty. }\end{array}$ & 1 & 2 & 3 & 4 & 5 \\
\hline $\begin{array}{l}\text { 7. I would like my hair to look like the } \\
\text { models who appear in magazines. }\end{array}$ & 1 & 2 & 3 & 4 & 5 \\
\hline $\begin{array}{l}\text { 8. I compare my appearance to the } \\
\text { appearance of TV and movie stars. }\end{array}$ & 1 & 2 & 3 & 4 & 5 \\
\hline $\begin{array}{l}\text { 9. Music videos on TV are not an } \\
\text { important source of information about hair } \\
\text { and "being attractive." }\end{array}$ & 1 & 2 & 3 & 4 & 5 \\
\hline $\begin{array}{l}\text { 10. I've felt pressure from } \mathrm{TV} \text { and } \\
\text { magazines to have a smoother hair texture. }\end{array}$ & 1 & 2 & 3 & 4 & 5 \\
\hline $\begin{array}{l}\text { 11. I would like my hair to look like the } \\
\text { people who are in movies. }\end{array}$ & 1 & 2 & 3 & 4 & 5 \\
\hline $\begin{array}{l}\text { 12. I do not compare my hair to the } \\
\text { textures/styles of people who appear in } \\
\text { magazines. }\end{array}$ & 1 & 2 & 3 & 4 & 5 \\
\hline
\end{tabular}


13. Magazine articles are not an important source of information about hair and "being attractive."

$\begin{array}{lllll}1 & 2 & 3 & 4 & 5\end{array}$

14. I've felt pressure from TV or magazines to have perfect hair.

15. I wish I looked like the models in music videos.

16. I compare my appearance to the appearance of people in magazines.

17. Magazine advertisements are an important source of information about hair and "being attractive."

1

18. I've felt pressure from TV or magazines to change my hair (texture/style/color).

1

19. I compare my hair to that of people with "good hair."

1

2

3

4

20. Pictures in magazines are an important source of information about hair and "being attractive."

1

2

3

4

5

21. I've felt pressure from TV, magazines, or social media to wear a weave or wig.

22. I compare my hair to that of people who have Afro hair.

12

3

4

5

23. Movies are an important source of information about hair and "being attractive."

12

3

4

5

24. I've felt pressure from TV or magazines to change my appearance.

1

25. I do not try to look like the people on TV.

26. Movie starts are not an important source of information about hairstyles and "being attractive."

1

23

$4 \quad 5$

1


27. Famous people are an important source of information about hairstyles and

28. I try to look like beauty Gurus on Social Media (YouTube, Instagram)

29. Pictures on social media (YouTube, Instagram, Pinterest, Facebook) are an important source of information about hair and "being attractive.

$\begin{array}{lllll}1 & 2 & 3 & 4 & 5\end{array}$

30. I've felt pressure from social media to have perfect hair.

$\begin{array}{lllll}1 & 2 & 3 & 4 & 5\end{array}$




\section{Internalized Racial Oppression Scale}

The following statements reflect some beliefs, opinions and attitudes. Read each statement carefully and give your honest feelings about the beliefs and attitudes expressed. Indicate the extent to which you agree or disagree using the following scale. There are no right or wrong answers. Please circle the number of that you feel best describes how you feel.

$1=$ Strongly Disagree

$2=$ Disagree

$3=$ Uncertain

$4=$ Agree

$5=$ Strongly Agree

\section{I wish I looked more White.}

12

3

4

5

2. There were universities and other learning centers in Africa more than 2000 years ago.

$\begin{array}{llllll}1 & 2 & 3 & 4 & 5\end{array}$

3. I would like a partner with lighter skin, to ensure that my children will have lighter skin.

4. Most criminals are Black men.

$\begin{array}{lllll}1 & 2 & 3 & 4 & 5\end{array}$

5. Straight hair is better than my natural hair texture.

1

23

$3 \quad 4 \quad 5$

6. African people have no written

\begin{tabular}{llllll} 
history. & 1 & 2 & 3 & 4 & 5 \\
\hline 7. Black women are confrontational. & 1 & 2 & 3 & 4 & 5
\end{tabular}

\begin{tabular}{llllll}
\hline $\begin{array}{l}\text { 8. The first mathematicians and } \\
\text { scientists were European. }\end{array}$ & 1 & 2 & 3 & 4 & 5 \\
\hline $\begin{array}{l}\text { 9. I prefer my hair to be natural. } \\
\text { 10. It is ok to straighten or relax my hair. }\end{array}$ & 1 & 2 & 3 & 4 & 5 \\
\hline
\end{tabular}

11. The earliest civilizations were in Africa.

$\begin{array}{lllll}1 & 2 & 3 & 4 & 5\end{array}$

12. Having full lips is not attractive to me.

13. Earlier Egyptians were either White or Arabic.

1

$\begin{array}{llll}2 & 3 & 4 & 5 \\ 2 & 3 & 4 & 5\end{array}$

1




\begin{tabular}{|c|c|c|c|c|c|}
\hline $\begin{array}{l}\text { 14. It is ok for Black people to change } \\
\text { their appearance through surgery. }\end{array}$ & 1 & 2 & 3 & 4 & 5 \\
\hline $\begin{array}{l}\text { 15. There were no institutions of higher } \\
\text { learning in ancient Africa. }\end{array}$ & 1 & 2 & 3 & 4 & 5 \\
\hline $\begin{array}{l}\text { 16. There were Africans in the Americas } \\
\text { prior to Europeans. }\end{array}$ & 1 & 2 & 3 & 4 & 5 \\
\hline 17. I wish my nose was narrower. & 1 & 2 & 3 & 4 & 5 \\
\hline 18. Black people are lazy. & 1 & 2 & 3 & 4 & 5 \\
\hline $\begin{array}{l}\text { 19. It is fine to use skin care products to } \\
\text { lighten skin color. }\end{array}$ & 1 & 2 & 3 & 4 & 5 \\
\hline $\begin{array}{l}\text { 20. Cannibalism was widely practiced in } \\
\text { Africa. }\end{array}$ & 1 & 2 & 3 & 4 & 5 \\
\hline $\begin{array}{l}\text { 21. I wish my skin was lighter than it is } \\
\text { now. }\end{array}$ & 1 & 2 & 3 & 4 & 5 \\
\hline 22. Black women are controlling. & 1 & 2 & 3 & 4 & 5 \\
\hline 23. I texturize my hair. & 1 & 2 & 3 & 4 & 5 \\
\hline $\begin{array}{l}\text { 24. Money management is something } \\
\text { that Black people cannot do. }\end{array}$ & 1 & 2 & 3 & 4 & 5 \\
\hline 25. Lighter sin is more attractive. & 1 & 2 & 3 & 4 & 5 \\
\hline 26. Most Black people are on welfare. & 1 & 2 & 3 & 4 & 5 \\
\hline 27. Black men are irresponsible. & 1 & 2 & 3 & 4 & 5 \\
\hline $\begin{array}{l}\text { 28. I like it when my partner wears his } \\
\text { or her hair natural. }\end{array}$ & 1 & 2 & 3 & 4 & 5 \\
\hline
\end{tabular}




\section{Multigroup Ethnic Identity Measure}

Use the numbers below to indicate how much you agree or disagree with each statement.

$1=$ Strongly Disagree

$2=$ Disagree

$3=$ Agree

$4=$ Strongly Agree

1. I have spent time trying to find out more about my ethnic group, such as its history, traditions, and customs.

$\begin{array}{llll}1 & 2 & 3 & 4\end{array}$

2. I am active in organizations or social groups that include mostly members of my own ethnic group.

$\begin{array}{llll}1 & 2 & 3 & 4\end{array}$

3. I have a clear sense of my ethnic background and what it means for me.

$\begin{array}{llll}1 & 2 & 3 & 4\end{array}$

4. I think a lot about how my life will be affected by my ethnic group membership.

5. I am happy that I am a member of the group I belong to.

$\begin{array}{llll}1 & 2 & 3 & 4\end{array}$

6. I have a strong sense of belonging to my own ethnic group.

$\begin{array}{llll}1 & 2 & 3 & 4\end{array}$

7. I understand pretty well what my ethnic group membership means to me.

$\begin{array}{llll}1 & 2 & 3 & 4\end{array}$

8. In order to learn more about my ethnic background, I have often talked to other people about my ethnic group.

9. I have a lot of pride in my ethnic group.

$\begin{array}{llll}1 & 2 & 3 & 4\end{array}$

\begin{tabular}{|c|c|c|c|c|}
\hline $\begin{array}{l}\text { 10. I participate in cultural practices of my own group, such } \\
\text { as special food, music, or customs. }\end{array}$ & 1 & 2 & 3 & 4 \\
\hline 11. I feel a strong attachment towards my own ethnic group. & 1 & 2 & 3 & 4 \\
\hline 12. I feel good about my cultural or ethnic background. & 1 & 2 & 3 & 4 \\
\hline
\end{tabular}




\section{General Self Efficacy Scale}

Use the numbers below to indicate how much you agree or disagree with each statement.

$1=$ Not at all true

2 = Hardly true

3 = Moderately true

$4=$ Exactly true

1. I can always manage to solve difficult problems if I try hard enough.

$1 \quad 2 \quad 3$

4

2. If someone opposes me, I can find the means and ways to get what I want.

$1 \quad 2 \quad 3$

4

3. It is easy for me to stick to my aims and accomplish my goals.

$1 \quad 2 \quad 3$

4

4. I am confident that I could deal efficiently with unexpected events.

1

5. Thanks to my resourcefulness, I know how to handle unforeseen situations.

1

6. I can solve most problems if I invest the necessary effort.

1

7. I can remain calm when facing difficulties because I can rely on my coping abilities.

9. If I am in trouble, I can usually think of a solution.

12

3

4

10 . I can usually handle whatever comes my way.

1

2

3

4 
APPENDIX E

OUTPUT FOR FITTED MODEL 


\section{Notes for Model (Default model)}

\section{Computation of degrees of freedom (Default model)}

Number of distinct sample moments: $\quad 66$

Number of distinct parameters to be estimated: 30

Degrees of freedom $(66-30)$ : $\quad 36$

\section{Result (Default model)}

Minimum was achieved

Chi-square $=76.726$

Degrees of freedom $=36$

Probability level $=.000$

\section{Maximum Likelihood Estimates}

\section{Regression Weights: (Group number 1 - Default model)}

\begin{tabular}{|c|c|c|c|c|c|c|}
\hline & & Estimate & S.E. & C.R. & $\mathrm{P}$ & Label \\
\hline IRO & $<---\quad$ MI & .398 & .053 & 7.438 & $* * *$ & \\
\hline EI & $<---\quad M I$ & .326 & .055 & 5.895 & $* * *$ & \\
\hline EI & $<---\quad$ IRO & -.188 & .055 & -3.389 & $* * *$ & \\
\hline Information & $<---\quad$ MI & 1.000 & & & & \\
\hline Pressure & $<---\quad M I$ & 1.187 & .078 & 15.283 & $* * *$ & \\
\hline Internalization Hair & $<---\quad$ MI & 1.069 & .061 & 17.379 & $* * *$ & \\
\hline Internalization Appearance & $<---\quad$ MI & 1.278 & .071 & 17.988 & $* * *$ & \\
\hline INS & $<---\quad$ IRO & 1.000 & & & & \\
\hline $\mathrm{BRH}$ & $<---\quad$ IRO & .600 & .053 & 11.336 & $* * *$ & \\
\hline APA & $<---\quad$ IRO & 1.063 & .067 & 15.972 & $* * *$ & \\
\hline $\mathrm{HC}$ & $<---\quad$ IRO & .585 & .064 & 9.207 & $* * *$ & \\
\hline EI Search & $<---\quad$ EI & 1.000 & & & & \\
\hline $\mathrm{EI} A B C$ & $<---\quad$ EI & 1.059 & .119 & 8.933 & $* * *$ & \\
\hline GSE & $<---\quad$ MI & -3.096 & .459 & -6.745 & $* * *$ & \\
\hline $\mathrm{EI} A B C$ & $<---\quad$ MI & -.402 & .056 & -7.183 & $* * *$ & \\
\hline GSE & $<---\quad$ EI & 5.897 & .741 & 7.963 & $* * *$ & \\
\hline
\end{tabular}

Standardized Regression Weights: (Group number 1 - Default model)

\begin{tabular}{|lrl|r|}
\hline & & & Estimate \\
\hline IRO & $<---$ & MI & .471 \\
EI & $<---$ & MI & .506 \\
EI & $<---$ & IRO & -.246 \\
Information & $<---$ & MI & .781 \\
Pressure & $<---$ & MI & .793 \\
Internalization Hair & $<---$ & MI & .877 \\
Internalization Appearance & $<---$ & MI & .904 \\
\hline
\end{tabular}




\begin{tabular}{|lcl|r|}
\hline & & & Estimate \\
\hline INS & $<---$ & IRO & .833 \\
BRH & $<---$ & IRO & .617 \\
APA & $<---$ & IRO & .886 \\
HC & $<---$ & IRO & .515 \\
EI Search & $<---$ & EI & .718 \\
EI ABC & $<---$ & EI & .919 \\
GSE & $<---$ & MI & -.446 \\
EI ABC & $<---$ & MI & -.540 \\
GSE & $<---$ & EI & .548 \\
\hline
\end{tabular}

Covariances: (Group number 1 - Default model)

\begin{tabular}{|lr|rrrrr|}
\hline & & Estimate & S.E. & C.R. & P & Label \\
\hline e2 $\langle-->$ & e13 & -.105 & .027 & -3.960 & $* * *$ & \\
e6 $\langle-->$ & e12 & -.071 & .016 & -4.512 & $* * *$ & \\
e5 $\langle-->$ & e11 & -.384 & .128 & -3.000 & .003 & \\
\hline
\end{tabular}

Correlations: (Group number 1 - Default model)

\begin{tabular}{|c|c|c|}
\hline & & Estimate \\
\hline $\mathrm{e} 2<-$ & $\begin{array}{ll}-\rightarrow & \text { e13 }\end{array}$ & -.271 \\
\hline e6 $<-$ & $-\rightarrow>$ e12 & -.335 \\
\hline e5 $<-$ & $-\rightarrow>$ e11 & -.208 \\
\hline
\end{tabular}

Variances: (Group number 1 - Default model)

\begin{tabular}{|l|rrrrr|}
\hline & Estimate & S.E. & C.R. & P & Label \\
\hline MI & .570 & .070 & 8.156 & $* * *$ & \\
e13 & .316 & .038 & 8.273 & $* * *$ & \\
e12 & .190 & .033 & 5.786 & $* * *$ & \\
e1 & .364 & .033 & 10.978 & $* * *$ & \\
e2 & .474 & .044 & 10.709 & $* * *$ & \\
e3 & .195 & .022 & 8.916 & $* * *$ & \\
$\mathrm{e} 4$ & .208 & .027 & 7.693 & $* * *$ & \\
e5 & .179 & .023 & 7.653 & $* * *$ & \\
$\mathrm{e} 6$ & .238 & .021 & 11.544 & $* * *$ & \\
$\mathrm{e} 7$ & .126 & .023 & 5.509 & $* * *$ & \\
$\mathrm{e} 8$ & .384 & .032 & 12.020 & $* * *$ & \\
$\mathrm{e} 9$ & .223 & .027 & 8.346 & $* * *$ & \\
$\mathrm{e} 10$ & .079 & .022 & 3.594 & $* * *$ & \\
$\mathrm{e} 11$ & 19.002 & 1.682 & 11.301 & $* * *$ & \\
\hline
\end{tabular}


Squared Multiple Correlations: (Group number 1 - Default model)

\begin{tabular}{|l|r|}
\hline & Estimate \\
\hline IRO & .222 \\
EI & .199 \\
GSE & .309 \\
EI ABC & .749 \\
EI Search & .515 \\
HC & .266 \\
APA & .784 \\
BRH & .380 \\
INS & .693 \\
Internalization Appearance & .818 \\
Internalization Hair & .770 \\
Pressure & .629 \\
Information & .610 \\
\hline
\end{tabular}

\section{Model Fit Summary}

\begin{tabular}{|l|rrrrr|}
\hline Model & NPAR & CMIN & $D F$ & $\mathrm{P}$ & CMIN/DF \\
\hline Default model & 30 & 76.726 & 36 & .000 & 2.131 \\
Saturated model & 66 & .000 & 0 & & \\
Independence model & 11 & 1767.952 & 55 & .000 & 32.145 \\
\hline
\end{tabular}

\begin{tabular}{|l|rrrr|}
\hline Model & RMR & GFI & AGFI & PGFI \\
\hline Default model & .069 & .958 & .923 & .522 \\
Saturated model & .000 & 1.000 & & \\
Independence model & .437 & .431 & .317 & .359 \\
\hline
\end{tabular}

\begin{tabular}{|l|rrrrr|}
\hline Model & $\begin{array}{r}\text { NFI } \\
\text { Delta1 }\end{array}$ & $\begin{array}{r}\text { RFI } \\
\text { rho1 }\end{array}$ & $\begin{array}{r}\text { IFI } \\
\text { Delta2 }\end{array}$ & $\begin{array}{r}\text { TLI } \\
\text { rho2 }\end{array}$ & \multirow{2}{*}{ CFI } \\
\hline Default model & .957 & .934 & .976 & .964 & .976 \\
Saturated model & 1.000 & & 1.000 & & 1.000 \\
Independence model & .000 & .000 & .000 & .000 & .000 \\
\hline
\end{tabular}

\begin{tabular}{|l|rrr|}
\hline Model & PRATIO & PNFI & PCFI \\
\hline Default model & .655 & .626 & .639 \\
Saturated model & .000 & .000 & .000 \\
Independence model & 1.000 & .000 & .000 \\
\hline
\end{tabular}

\begin{tabular}{|l|rrr|}
\hline Model & NCP & LO 90 & HI 90 \\
\hline Default model & 40.726 & 19.301 & 69.899 \\
Saturated model & .000 & .000 & .000 \\
Independence model & 1712.952 & 1579.347 & 1853.924 \\
\hline
\end{tabular}




\begin{tabular}{|l|rrrr|}
\hline Model & NCP & & LO 90 & HI 90 \\
\hline Model & FMIN & F0 & LO 90 & HI 90 \\
\hline Default model & .239 & .127 & .060 & .218 \\
Saturated model & .000 & .000 & .000 & .000 \\
Independence model & 5.508 & 5.336 & 4.920 & 5.775 \\
\hline
\end{tabular}

\begin{tabular}{|l|rrrr|}
\hline Model & RMSEA & LO 90 & HI 90 & PCLOSE \\
\hline Default model & .059 & .041 & .078 & .189 \\
Independence model & .311 & .299 & .324 & .000 \\
\hline
\end{tabular}

\begin{tabular}{|l|rrrr|}
\hline Model & AIC & BCC & BIC & CAIC \\
\hline Default model & 136.726 & 139.056 & 249.962 & 279.962 \\
Saturated model & 132.000 & 137.126 & 381.120 & 447.120 \\
Independence model & 1789.952 & 1790.806 & 1831.472 & 1842.472 \\
\hline
\end{tabular}

\begin{tabular}{|l|rrrr|}
\hline Model & ECVI & LO 90 & HI 90 & MECVI \\
\hline Default model & .426 & .359 & .517 & .433 \\
Saturated model & .411 & .411 & .411 & .427 \\
Independence model & 5.576 & 5.160 & 6.015 & 5.579 \\
\hline
\end{tabular}

\begin{tabular}{|l|rr|}
\hline \multirow{2}{*}{ Model } & HOELTER & HOELTER \\
& .05 & .01 \\
\hline Default model & 214 & 246 \\
Independence model & 14 & 15 \\
\hline
\end{tabular}


REFERENCE LIST 


\section{REFERENCE LIST}

Aliaga, M., \& Gunderson, B. (2000). Introduction to quantitative research. Doing quantitative research in education with SPSS. Thousand Oaks, CA: Sage Publications.

Allen, M. (2016, December 05). Model Duckie Thot speaks out about natural hair. Retrieved from https://www.yahoo.com/lifestyle/model-duckie-thot-speaksnatural-170944501.html

Allen, R. L. (2001). The concept of self: A study of Black identity and self-esteem. Detroit, MI: Wayne State University Press.

Andrews, H. (2014). The great hair dilemma. Essence, 44(9), 48-49.

Arogundade, B. (2000). Black beauty: A history and a celebration. London, England: Thunder's Mouth Press.

Azibo, D. A. (1989). African-centered theses on mental health and a nosology of Black/African personality disorder. The Journal of Black Psychology, 15, 173 214.

Badillo, C. (2001). "Only my hairdresser knows for sure.” Stories of race, hair and gender. NACLA Report on the Americas, 34, 35-38.

Bailey, T. K. M., Chung, Y. B., Williams, W. S., Singh, A. A., \& Terrell, H. K. (2011). Development and validation of the Internalized Racial Oppression Scale for Black individuals. Journal of Counseling Psychology, 58(4), 481.

Bandura, A. (1977). Self-efficacy: Toward a unifying theory of behavioral change. Psychological Review, 84(2), 191-215.

Bandura, A. (1988). Organizational application of social cognitive theory. Australian Journal of Management, 13(2), 275-302.

Banks, I. (2000). Hair matters: Beauty, power, and Black women's consciousness. New York, NY: New York University Press. 
Berry, G. L. (1998). Black family life on television and the socialization of the African American child: Images of marginality. Journal of Comparative Family Studies, 29(2), 233-242.

Bond, S., \& Cash, T. F. (1992). Black beauty: Skin color and body images among African-American women. Journal of Applied Social Psychology, 22, 874-888.

Bonner, L. B. (1991). Good hair: For colored girls who've considered weaves when the chemicals became too Ruff. New York, NY: Crown Trade Paperbacks.

Botta, R. A. (2000). The mirror of television: A comparison of Black and White adolescents' body image. Journal of Communication, 50(3), 144-159.

Boyd-Franklin, N. (1991). Recurrent themes in the treatment of African-American women in group psychotherapy. Women \& Therapy, 11, 25-40.

Brown, K. (1998). Consequences of skin tone bias for African Americans: Resource attainment and psychological/social functioning. Perspectives, 4, 95-104.

Bryman, A., Teevan, J., \& Bell, E. (2005). Chapter 13: Qualitative data analysis. In Bryman \& Teevan, Social research methods ( $3^{\text {rd }}$ ed.), (pp. 282-308). Oxford, England: Oxford Press.

Buchanan, D. R. (1993). Social status group differences in motivations for drug use. Journal of Drug Issues, 23(4), 631-644.

Byrd, A. D., \& Tharps, L. L. (2001). Hair story: Untangling the roots of Black hair in America. New York, NY: St. Martin's Press.

Capodilupo, C. M. (2010). The effects of idealized media images on the body esteem and appearance satisfaction of African American women (Doctoral dissertation). Available from ProQuest PsycINFO database. (Order No. AAI3386128).

Chambers, J. W., Clark, T., Dantzler, I., \& Baldwin, J.A. (1994). Perceived attractiveness, facial features, and African self-consciousness. Journal of Black Psychology, 20, 305-324.

Clark, K. B., \& Clark, M. K. (1939). The development of consciousness of self and the emergence of racial identification in Negro preschool children. The Journal of Social Psychology, 10(4), 591-599.

Clark, K. B., \& Clark, M. K. (1940). Skin color as a factor in racial identification of Negro preschool children. The Journal of Social Psychology, 11(1), 159-169. 
Cleage, P. (1993). Hairpeace. African American Review, 27, 37-41.

Collins, P. H. (2002). Black feminist thought: Knowledge, consciousness, and the politics of empowerment. New York, NY: Routledge.

Cross, W. E. (1994). Nigrescence theory. Historical and explanatory notes. Journal of Vocational Behavior, 44, 119-123.

Dossou, M. (2013, July 03). Natural hair vs. corporate America: Why are we still fighting this battle? Retrieved from http://www.ebony.com/style/fighting-for-ourhair-in-corporate-america- 032

Du Bois, W. E. (1903). 1989. The souls of black folk. Saint Louis, MO: Turtleback Books.

Ebony (1996, May). The ebony advisor. Ebony Magazine, 64.

Entman, R. M., \& Rojecki, A. (2001). The black image in the white mind: Media and race in America. Chicago, IL: University of Chicago Press.

Ethnicity. (n.d.). In Merriam Webster Online. Retrieved from https://www.merriamwebster.com

Ferrell, P. (1996). Let's talk hair: Every woman's personal consultation for healthy growing hair. Washington, DC: Cornrows \& Co.

Fisher, M. (2013, May 16). A revealing map of the world's most and least ethnically diverse countries. Retrieved from https://www.washingtonpost.com/news/worldviews/wp/2013/05/16/a-revealingmap-of-the-worlds-most-and-least-ethnically-diverse-countries/?noredirect=on

Freedman, R. (1988). Beauty bound: Why women strive for physical perfection. London, England: Columbus.

Frisby, C. M. (2004). Does race matter? Effects of idealized images on African American women's perceptions of body esteem. Journal of Black Studies, 34(3), 323-347.

Fujioka, Y. (2005). Black media images as a perceived threat to African American ethnic identity: Coping responses, perceived public perception, and attitudes towards affirmative action. Journal of Broadcasting \& Electronic Media, 49(4), 450-467. 
Gaskins, B. (1997). Good and bad hair: Photographs by Bill Gaskins. New Brunswick, NJ: Rutgers University Press.

Gatewood, W. B. (1988). Aristocrat of color: South and north and the Black elite, 1880-1930. Journal of Southern History, 54, 3-19.

Gibson, C. (2017, November 13). Lupita Nyong'o on the Grazia UK photoshop natural hair controversy. Retrieved from https://www.usmagazine.com/stylish/news/lupita-nyongo-on-the-grazia-ukphotoshop-natural-hair-controversy/

Greene, B., White, J. C., \& Whitten, L. (2000). Hair texture, length, and style as a metaphor in the African American mother-daughter relationship: Considerations in psychodynamic psychotherapy. In L. C. Jackson, \& B. Green (Eds.), Psychotherapy with African-American women: Innovations in psychodynamic perspectives and practice (pp.166-193). New York, NY: Guilford Press.

Gottschall, J. (2008). The "beauty myth" is no myth: Emphasis on male-female attractiveness in world folktales. Human Nature, 19, 174-188.

Grier, W., \& Cobbs, P. (1980). Black rage (2 ${ }^{\text {nd }}$ ed.). New York, NY: Basic Books.

Hall, C. C. I. (1995). Beauty is in the soul of the beholder: Psychological implications of beauty and African American women. Cultural Diversity and Mental Health, 1(2), 125.

Hall, R. (1995). The bleaching syndrome: African Americans' response to cultural domination vis-à-vis skin color. Journal of Black Studies, 26(2), 172-184.

Hall, S. (1986). Gramsci's relevance for the study of race and ethnicity. The Journal of Communication Inquiry, 10(2), 5-27.

Harrison, M. S., \& Thomas, K. M. (2009). The hidden prejudice in selection: A research investigation on skin color bias. Journal of Applied Social Psychology, $39,134-168$.

Helms, J. E. (1990). Black and White racial identity: Theory, research, and practice. Westport, CT: Greenwood Press.

Helms, J. E. (1994). The conceptualization of racial identity and other" racial" constructs. In E. J. Trickett, R. J. Watts, \& D. Birman (Eds.), Human diversity: Perspectives on people in context (pp. 285-311). San Francisco, CA: Jossey-Bass 
Helms, J. E. (1995). An update of Helm's White and people of color racial identity models. In J. G. Ponterotto, J. M. Casas, L. A. Suzuki, \& C. M. Alexander (Eds.), Handbook of multicultural counseling (pp. 181-198). Thousand Oaks, CA, US: Sage Publications, Inc.

Herring, C. (2004). Skin deep: Race and complexion matter in the "color-blind" era. In C. Herring, V. M. Keith, \& D. H. Horton (Eds.), Skin deep: How race and complexion matter in the "color-blind" era (pp. 1-21). Chicago, IL: University of Illinois Press.

Hochschild, J. L. (2007). The skin color paradox and the American racial order. Social Forces, 86, 643-670.

Hill, R.B. (1999). The strengths of African-American families: Twenty-five years later. Lanham, MD: University Press of America, Inc.

Hu, L., \& Bentler, P. M. (1999). Cutoff criteria for fit indexes in covariance structure analysis: Conventional criteria versus new alternatives. Structural Equation Modeling, 6, 1-55.

Hughes, M. \& Hertel, B.R. (1990). The significance of color remains: A study of life chances, mate selection, and ethnic consciousness among Black Americans. Social Forces, 68, 1105-1120.

Hunter, A. G., \& Sellers, S. L. (1998). Feminist attitudes among African American women and men. Gender \& Society, 12(1), 81-99.

Hunter, M. (2004). Light, bright, and almost white: The advantages and disadvantages of light skin. In C. Herring, V. M. Keith, \& H. D. Horton (Eds.), Skin deep: How race and complexion matter in the "color-blind" era (pp. 22-44). Chicago, IL: University of Illinois Press.

Hunter, M. L. (1998). Colorstruck: Skin color stratification in the lives of African American women. Sociological Inquiry, 68, 517-535.

Hunter, M. L. (1998). Colorstruck: Skin color stratification in the lives of African American women. Sociological Inquiry, 68, 517-535.

Hunter, M. L. (2013). Race, gender, and the politics of skin tone. New York, NY: Routledge. 
Imarogbe, K. (2003). Hair misorientation: Free your mind and your hair will follow. In D. Azibo (Ed.), African-centered psychology: Culture-focusing for multicultural competence (pp. 201-220). Durham, NC: Carolina Academic Press.

Imarogbe, K. A. (2003). Perceived racism, afrocentrism, and black racial identity as predictors of body dissatisfaction in Black Americans (Doctoral dissertation). Available from ProQuest Dissertations and Theses database. (Order No. 3111476).

James-Cue, M. (2011). Media images of African American women and African American adolescent girls' self-esteem and body esteem (Doctoral dissertation). Available from ProQuest Dissertations and Theses database. (Order No. 3500212).

Kambon, K. K. (1992). The African personality in America: An African-centered framework. Tallahassee, FL: Nubian Nation.

Kaw, E. (1994). "Opening faces": The politics of cosmetic surgery and Asian American women. In N. Sault (Ed.), Many mirrors: Body image and social relations (pp. 241-265). New Brunswick, NJ: Rutgers University Press.

Khew, J. (2016, August 26). Mass media influences on the standards of beauty in the society. Retrieved from https://www.linkedin.com/pulse/mass-mediainfluences-standards-beauty-society-jolyn-khew

Kellner, D. (1995). Media communications vs. cultural studies: Overcoming the divide. Communication Theory, 5(2), 162-177.

Kenyatta, K., \& Kenyatta, J. (1996). Black folk's hair: Secrets, shame and liberation. Albrightsville, PA: Songhai.

Kerr, A. E. (2005). The paper bag principle: Of the myth and the motion of colorism. Journal of American Folklore, 118, 271-289.

Lakoff, R.T. \& Scherr, R.L. (1984). Face value. Politics of beauty. Boston, MA: Kegan \& Paul.

Leeds, M. (1994). Young African-American women and the language of beauty. Contributions in Women's Studies, 141, 147-147. 
Loechner, J. (2015, February 23). African-Americans top media users. Retrieved from https://www.mediapost.com/publications/article/244244/african-americanstop-media-users.html

Luszczynska, A., \& Schwarzer, R. (2005). Social cognitive theory. In M. Conner \& P. Norman (Eds.), Predicting health behavior (2nd ed. rev., pp. 127-169). Buckingham, England: Open University Press.

Makkar, J. K., \& Strube, M. J. (1995). Black women's self-perceptions of attractiveness following exposure to White versus Black beauty standards: The moderating role of racial identity and self-esteem. Journal of Applied Social Psychology, 25(17), 1547-1566.

Martin, J. G. (1964). Racial ethnocentrism and judgment of beauty. Journal of Social Psychology, 63, 59-63.

Mason, A. C. (2016). Hair shame: Multigenerational transmission of internalized racism in African American women (Doctoral dissertation). Available from ProQuest Dissertation and Theses database, PsycINFO. (Order No. AAI3700913).

McCombs, M. E., \& Shaw, D. L. (1972). The agenda-setting function of mass media. Public Opinion Quarterly, 36(2), 176-187.

Mercer, K. (1990). Black hair/style politics. In R. Ferguson, M. Gever, T. T. Minh-ha, \& C. West (Eds.), Out there: Marginalization and contemporary cultures (pp. 247264). New York, NY: The New Museum of Contemporary Art and The MIT Press.

Meyers, L. S., Gamst, G., \& Guarino, A. J. (2013). Applied multivariate research: Design and interpretation. Thousand Oaks, CA: Sage Publications.

Miller, N. E., \& Dollard, J. (1941). Social learning and imitation. New Haven, CT: Yale University Press.

Mischel, W., Shoda, Y. (1995). A cognitive-affective system theory of personality: Reconceptualizing situations, dispositions, dynamics, and invariance in personality structure. Psychological Review, 102, 246-268.

Montalvo, F. F. (2004). Surviving race: Skin color and the socialization and acculturation of Latinas. Journal of Ethnic \& Cultural Diversity in Social Work, $13,25-43$.

Myers, L. J. (1993). Understanding an Afrocentric world view: Introduction to an optimal psychology. Dubuque, IA: Kendall/Hunt Publishing. 
NAACP Legal Defense Fund: Defend, Educate, Empower. (n.d.). Retrieved from http://www.naacpldf.org/brown-at-60-the-doll-test

Neal, A. M., \& Wilson, M. L. (1989). The role of skin color and features in the Black community: Implications for Black women and therapy. Clinical Psychology Review, 9, 323-333.

Oliveira, M. (2000). The effect of media's objectification of beauty on children's body esteem (Doctoral dissertation). Brandeis University, Waltham, MA.

Onwuachi-Willig, Angela. (2016, September 6). Race and racial identity are social constructs. The New York Times. Retrieved from https://www.nytimes.com

Ormrod, J. E. (2006). Educational psychology: Developing learners (5th ed.). Upper Saddle River, NJ: Pearson/Merrill Prentice Hall.

Padilla, L. M. (2001). But you're not a dirty Mexican: Internalized oppression, Latinos \& law. Texas Hispanic Journal of Law and Policy, 7(1), 59-113.

Parks, C. W., \& Woodson, K. M. (2002). The impact of skin color and hair texture on mate selection: Implications for interventions with African American men and women. In E. Davis-Russell (Ed.), The California school of professional handbook on multicultural education, research, intervention, and training (pp. 249-262). San Francisco, CA: Jossey Bass.

Patton, T. O. (2006). Hey girl, am I more than my hair?: African American women and their struggles with beauty, body image, and hair. NWSA, 18, 25-51.

Phinney, J. S. (1992). The multigroup ethnic identity measure: A new scale for use with diverse groups. Journal of Adolescent Research, 7(2), 156-176.

Price, J. H., \& Murnan, J. (2004). Research limitations and the necessity of reporting them. American Journal of Health Education, 35, 66-67.

Pyke, K. D. (2010). What is internalized racial oppression and why don't we study it? Acknowledging racism's hidden injuries. Sociological Perspectives, 53(4), 551572.

Race. (n.d.). In Merriam Webster Online. Retrieved from https://www.merriamwebster.com

Rhodan, M. (2014, August 13). Military rolls back restrictions on Black hair styles. Retrieved from http://time.com/3107647/military-black-hairstyles/ 
Riggott, J. (2013, October 13). Americans consume media in a major way, study finds. Retrieved from https://news.usc.edu/56894/americans-consume-media-in-amajor-way-study-finds/

Roberts, R. E., Phinney, J. S., Masse, L. C., Chen, Y. R., Roberts, C. R., \& Romero, A. (1999). The structure of ethnic identity of young adolescents from diverse ethnocultural groups. The Journal of Early Adolescence, 19(3), 301-322.

Robinson, C. L. (2011). Hair as race: Why "good hair" may be bad for Black females. Howard Journal of Communications, 4, 358-376.

Robinson, T. L., \& Ward, J. V. (1995). African American adolescents and skin color. Journal of Black Psychology, 21, 256-274.

Rooks, N. (1996). Hair raising: Beauty, culture, and African American women. New Brunswick, NJ: Rutgers University Press.

Rosenthal, J. A. (1996). Qualitative descriptors of strength of association and effect size. Journal of Social Service Research, 21(4), 37-59.

Ruddock, A. (2014, December 18). Hair type classification. Retrieved from https://blackhairinformation.com/general-articles/hair-type-chart discover-hairtype/

Russell, K., Wilson, M., \& Hall, R. (1992). The color complex: The politics of skin color among African-Americans. New York, NY: Anchor Doubleday.

Salkind, N. J. (2011). Ice cream and crime: Computing correlation coefficients. In N. J. Salkind, Statistics for people who think they hate statistics, (pp. 113-136). Thousand Oaks, CA: Sage.

Sellers, R. M., Smith, M. A., Shelton, J. N., Rowley, S. A., \& Chavous, T. M. (1998). Multidimensional model of racial identity: A reconceptualization of African American racial identity. Personality and Social Psychology Review, 2(1), 18-39.

Schooler, D., Monique Ward, L., Merriwether, A., \& Caruthers, A. (2004). Who's that girl: Television's role in the body image development of young white and black women. Psychology of Women Quarterly, 28(1), 38-47.

Schwalbe, M., Holden, D., Schrock, D., Godwin, S., Thompson, S., \& Wolkomir, M. (2000). Generic processes in the reproduction of inequality: An interactionist analysis. Social Forces, 79(2), 419-452. 
Schwarzer, R., \& Jerusalem, M. (1995). Generalized self-efficacy scale. In J. Weinman, S. Wright, \& M. Johnston, Measures in health psychology: A user's portfolio. Causal and control beliefs (pp. 35-37). Windsor, United Kingdom: NFERNELSON.

Sciarra, D. T., \& Gushue, G. V. (2003). White racial identity development and religious orientation. Journal of Counseling \& Development, 81(4), 473-482.

Shakur, A. (1987). Assata: An autobiography. West Port, CT: Lawrence Hill \& Co.

Spellers, R. E. (2003). The kink factor: A womanist discourse analysis of AfricanAmerican mother/daughter perspectives on negotiating Black hair/body politics. In R. L. Jackson II \& E. B. Richardson (Eds.), Understanding African-American rhetoric: Classical origins to contemporary innovations (pp. 223-243). New York, NY: Routledge.

Starr, T. J. (2014, February 08). Weather woman fired after defending natural hair and Black kids has no regrets. Retreived from https:// newsone.com/2867261/rhonda-lee-weather-woman-fired-natural-hair/

Synnott, A. (1987). Shame and glory: A sociology of hair. British Journal of Sociology, $38,381-413$.

Tajfel, H. (1982). Social psychology of intergroup relations. Annual Review of Psychology, 33(1), 1-39.

Taylor, P. C. (1999). Malcolm's conk and Danto's colors: Or, four logical petitions concerning race, beauty and aesthetics. Journal of Aesthetics \& Art Criticism, 57, 16-21.

Thomas, S. I. (2006). African American adolescent females: An investigation of racial identity, skin color and self-concept during adolescent development (Doctoral dissertation). Kent State University, Kent, $\mathrm{OH}$.

Thompson, J. K., van den Berg, P., Roehrig, M., Guarda, A. S., \& Heinberg, L. J. (2004). The sociocultural attitudes towards appearance scale-3 (SATAQ-3): Development and validation. International Journal of Eating Disorders, 35(3), 293-304.

Thompson, M. S., \& Keith, V. M. (2004). Copper brown and blue black: Colorism and self evaluation. In C. Herring, V. M. Keith, \& D. H. Horton (Eds.), Skin deep: How race and complexion matter in the "color-blind" era (pp. 45-64). Chicago, IL: University of Illinois Press. 
Trevino, A. J., Harris, M. A., \& Wallace, D. (2008). What's so critical about critical race theory? Contemporary Justice Review, 11, 7-10.

Vandiver, B. J., Cross Jr, W. E., Worrell, F. C., \& Fhagen-Smith, P. E. (2002). Validating the Cross Racial Identity Scale. Journal of Counseling Psychology, 49(1), 71.

Vargas, N., \& Kingsbury, J. (2016). Racial identity contestation: Mapping and measuring racial boundaries. Sociology Compass, 10(8), 718-729.

Wade, T. J. (1996). The relationship between skin color and self-perceived global, physical and sexual attractiveness, and self-esteem for African Americans. Journal of Black Psychology, 21, 358-373.

Ward, L. M. (2004). Wading through the stereotypes: positive and negative associations between media use and black adolescents' conceptions of self. Developmental Psychology, 40(2), 284.

Weitz, R. (2004). Rapunzel's daughters: What women's hair tells us about women's lives. New York, NY: Farrar, Strauss, and Giroux.

West, C. M. (1995). Mammy, Sapphire, and Jezebel: Historical images of Black women and their implications for psychotherapy. Psychotherapy: Theory, Research, Practice, Training, 32(3), 458.

White, T. R. (2008). Media as pedagogy and socializing agent: Influences of feminine beauty aesthetics in American teen-oriented films and magazines on African American adolescent female self image (Doctoral dissertation). Available from ProQuest Dissertations and Theses Global. (Order No. 3332535).

Wolf, N. (2002). The beauty myth: How images of beauty are used against women. New York, NY: HarperCollins.

Woodard, J. B., \& Mastin, T. (2005). Black womanhood: Essence and its treatment of stereotypical images of Black women. Journal of Black Studies, 36(2), 264-281.

Woodson, K. M. (2002). The impact of hair texture and skin color among African American men and women during mate selection on the expression of risky sexual behaviors (immune deficiency) (Doctoral dissertation). Available from ProQuest Dissertations and Theses database. (Order No. AAI3043493).

Woolford, S. J., Woolford-Hunt, C. J., Sami, A., Blake, N., \& Williams, D. R. (2016). No sweat: African American adolescent girls' opinions of hairstyle choices and physical activity. BMC obesity, 3(1), 31. 
Wyatt, G. E. (1977). Stolen women: Reclaiming our sexuality, taking back our lives. New York, NY: Wiley.

Yap, S. C., Donnellan, M. B., Schwartz, S. J., Kim, S. Y., Castillo, L. G., Zamboanga, B. L., \& Vazsonyi, A. T. (2014). Investigating the structure and measurement invariance of the multigroup ethnic identity measure in a multiethnic sample of college students. Journal of Counseling Psychology, 61(3), 437. 
NAME: $\quad$ Kristy L. La Mar

PLACE OF BIRTH: Rockville Center, New York

EDUCATION: $\quad$ Ph.D., Educational Psychology, 2018

Andrews University, Berrien Springs, Michigan

M.S., Counseling Psychology, 2016

Andrews University, Berrien Springs, Michigan

M.A., General Psychology, 2013

Adelphi University, Garden City, New York

B.S., English, 2005

Oakwood University, Huntsville, Alabama

A.S., Photography, 2005

Oakwood University, Huntsville, Alabama

\section{PROFESSIONAL EXPERIENCE}

2018

2017

2016

$2015-2016$
Adjunct Professor

Oakwood University, Department of Psychological Sciences

Huntsville, Alabama

Ph.D. Internship Counselor

University of Alabama in Huntsville

Huntsville, Alabama

M.A. Practicum Supervisor

Andrews Community Counseling Center

Berrien Springs, Michigan

Ph.D. Practicum Counselor

Andrews Community Counseling Center

Berrien Springs, Michigan 